\title{
Screening for fetal trisomy 21 in North-Belgium
}

\author{
Citation for published version (APA):
}

Gyselaers, W. J. (2005). Screening for fetal trisomy 21 in North-Belgium. [Doctoral Thesis, Maastricht University]. https://doi.org/10.26481/dis.20051206wg

Document status and date:

Published: 01/01/2005

DOI:

10.26481/dis.20051206wg

Document Version:

Publisher's PDF, also known as Version of record

\section{Please check the document version of this publication:}

- A submitted manuscript is the version of the article upon submission and before peer-review. There can be important differences between the submitted version and the official published version of record.

People interested in the research are advised to contact the author for the final version of the publication, or visit the DOI to the publisher's website.

- The final author version and the galley proof are versions of the publication after peer review.

- The final published version features the final layout of the paper including the volume, issue and page numbers.

Link to publication

\footnotetext{
General rights rights.

- You may freely distribute the URL identifying the publication in the public portal. please follow below link for the End User Agreement:

www.umlib.nl/taverne-license

Take down policy

If you believe that this document breaches copyright please contact us at:

repository@maastrichtuniversity.nl

providing details and we will investigate your claim.
}

Copyright and moral rights for the publications made accessible in the public portal are retained by the authors and/or other copyright owners and it is a condition of accessing publications that users recognise and abide by the legal requirements associated with these

- Users may download and print one copy of any publication from the public portal for the purpose of private study or research.

- You may not further distribute the material or use it for any profit-making activity or commercial gain

If the publication is distributed under the terms of Article $25 \mathrm{fa}$ of the Dutch Copyright Act, indicated by the "Taverne" license above, 
(1) Willried Gyselaers, Genk 2005

ISBN: $\quad 90-9020048-7$

Lay-out: Patricia Jarkowski

Printing: Genk Druk, Zuiderring 45, 3600 Genk

Publication of this thesis was financially supported by GIFT vzw Genk, with special thanks to: Hitachi Medical Ultrasound, Aloka BioMedic, Esaote, Philips Medical Systems, Toshiba Medical Systems. 


\title{
Screening for fetal trisomy 21 in North - Belgium.
}

\author{
Proefschrift \\ ter verkrijging van de graad van doctor \\ aan de Universiteit Maastricht \\ op gezag van de Rector Magnificus \\ Prof. mr. G.P.M.F. Mols, \\ volgens het besluit van het College van Decanen, \\ in het openbaar te verdedigen op \\ dinsdag 6 december 2005 om 16.00 uur.
}

door

Wilfried Jozef Albert Gyselaers 


\section{Promotor}

Prof. dr. J.G. Nijhuis

\section{Copromotores}

Dr. W. Ombelet (Ziekenhuis Oost Limburg, Genk, België)

Dr. I. Witters (Katholieke Universiteit Leuven, België)

\section{Beoordelingscommissie}

Prof. dr. J.P.M. Geraedts (voorzitter)

Prof. dr. M. van Dieijen-Visser

Prof. dr. N. Wald (Wolfson Institute of Preventive Medicine, London)

Prof. dr. G.M.W.R. de Wert

The author did not receive any grants, financial support or material benefits from Algemeen Medisch Laboratorium AML in Antwerp or other institutions, before, during or after this study. The AML-algorithms for fetal aneuploidy screening have not been patented. 
Dedicated to

Amaat Van Waes († 10/8/2002),

my very first teacher of obstetrics. 



\section{Contents}

p. 11. Abbreviations

p. 12. Definitions

p. 15. Chapter 1. General introduction.

Adapted from :

"Trisomiescreening: een kritische evaluatie en prakrische adviezen."

Tijdschift woor Geneeskunde, 2003;59:1445-54.

p. 41. Chapter 2. Fetal aneuploidy screening in Belgium between 1995 and 2001.

Aims of the study.

p. 49. Chapter 3. Materials and methods:

3.1. Basic principles of screening.

3.2. The fetal aneuploidy screening program, organised by AML Antwerp.

p. 73. Chapter 4. Screening for trisomy 21 in Flanders: a 10-years review of 40490 pregnancies screened by maternal serum.

Eur J Obstet Gynecol Reprod Biol 2004; 115:185-9.

p. 87. Chapter 5. Single-step maternal serum screening for trisomy

21 in the era of combined or integrated screening.

Gynecol Obstet Invest 2004;58:221-4.

p. 99. Chapter 6. Audit on nuchal translucency thickness measurements in Flanders, Belgium: a plea for methodologic standardisation.

Ultrasound Obstet Gynecol 2004;24:511-5. 
p. 117. Chapter 7. First trimester ultrasound screening in Flanders, Belgium: - survey on the future organisation - the program for training and quality control

Adapted from:

"Survey on the future organisation of first trimester ultrasound screening in Flanders, Belgium."

Prenatal Diagnosis 2005;25:518-9.

p. 125. Chapter 8. Population screening for fetal trisomy 21: easy access to screening should be balanced against a uniform ultrasound protocol.

Prenatal Diagnosis (in press).

p. 145. Chapter 9. Nuchal translucency thickness measurements for fetal aneuploidy screening: LogNT-MoM or Delta-NT, performer-specific medians or ultrasound training.

Submitted.

p. 161. Chapter 10. Fetal aneuploidy screening practice in Flanders and Belgium.

In: Gynaecology, Obstetrics and Reproductive Medicine in daily practice. Slager $E_{n}$ Fauser $B$, van Geijn $H$, Brölman $\mathrm{H}$, Vervest $H$ (eds).

International Congress Series 2005;1279:228-33.

p. 173. Chapter 11. General discussion and future perspectives.

p. 185. Chapter 12. Summary.

p. 193. Chapter 13. Samenvatting.

p. 201. Nawoord en dank

p. 207. Curriculum Vitae

p. 209. Publicaties en Abstracts 


\section{Abbreviations}

IMSS

2MSS

AC

AFP

$\mathrm{CM}$

CRL

CVs

$\Delta$-value (Delta-value)

DR

FB-HCG

FK

FPR

HCG

MA

MoM

MSS

NB

NT

PAPP-A

RIZIV

SPR

T21

$\mathrm{uE}_{3}$
Maternal serum screening in the first trimester

(PAPP-A + FB-HCG)

Maternal serum screening in the second

trimester (AFP, $\mathrm{HCG}+\mathrm{uE}_{3}$ )

Amniocentesis

Alfa-Foeto Protein

Christelijke Mutualiteiten

Crown Rump Length

Chorionic villus sampling

Difference between the measured value and the corresponding median

Detection rate

Free B-Human Chorionic Gonadotropin

Fetal karyotyping

False positive rate

Human Chorionic Gonadotropin

Maternal age

Multiple of the Median

Maternal serum screening

Nasal bone

Nuchal Translucency thickness

Pregnancy Associated Plasma Protein A

Rijksinstifuut voor Ziekte- en

Invaliditeitsverzekering

Screen positive rate

Trisomy 21

Unconjugated estriol 


\section{Definitions}

Aneuploidy

Abnormal number of chromosomes, different from $46 \mathrm{XX}$ or $46 \mathrm{XY}$. This may be due to one extra chromosome (trisomy) or chromosomes (polyploidy) or due to a missing chromosome (monosomy).

Combined screening

Screening using both maternal serum and ultrasound parameters.

Cut off level

Detection rate

The level chosen to define a positive screening result and distinguish it from a negative result.

False positive rate

Fetal loss

First trimester

Integrated screening

Maternal serum screening

Negative screening result
Number of affected pregnancies, detected by

the screening test, over the total number of affected pregnancies in the population.

Number of unaffected pregnancies with a positive screening result, over the total number of unaffected pregnancies in the population.

Pregnancy that has miscarried or resulted in an intra-uterine death or stillbirth.

$\leq 13^{+6}$ weeks of gestation.

Screening using maternal serum and ultrasound parameters, sampled in both the first trimester and second trimester.

Screening using maternal age and serum parameters.

Screening result below the cut off level, due to which the pregnancy is not considered at risk for fetal aneuploidy. 
Positive screening result

Second trimester

Screen positive rate

Ultrasound screening
Screening result above the cut off level, due to which the pregnancy is considered at risk for fetal aneuploidy.

Between $14^{+0}$ and 28 weeks of gestation

The number of positive screening results over the total number of screening tests.

Screening using maternal age and ultrasound parameters. 


\section{Chapter 1}

\section{General introduction}

Adapted from:

Gyselaers WJA, Vereecken AJ, Van Herck EJH, Straetmans DPL, De Jonge ETM.

Onbelet WUAM.

Trisomiescreening: een kritische evaluatie en praktische adviezen.

Tijdschrift woor Geneeskunde 2003;59:1445-1454. 


\section{Summary}

The history of fetal aneuploidy screening is summarised. The characteristics of maternal serum and ultrasound screening are discussed, and the different screening methods are compared: the most sensitive and specific methods today are (1) the combination of first trimester fetal nuchal translucency measurement with maternal serum biochemistry in the first or second trimester and (2) the integrated first and second trimester screening test. Audit and quality control of the different methods used are crucial to guarantee the quality of the screening programs. The organisation of fetal aneuploidy screening programs using serum and/or ultrasound parameters is considered cost-beneficial. For medical, ethical and economical reasons, it is recommended to abandon a screening policy by invasive testing for advanced maternal age only. Procedure related risk for fetal loss due to first trimester chorionic villus sampling or second trimester amniocentesis is estimated to be around 0.5-1\%. Patient counselling is of major importance and recommended before any screening test is done. Some new developments expected for the near future are discussed briefly. 


\subsection{History of fetal aneuploidy screening.}

The clinical features of one specific type of mental retardation were described by Langdon Down in 1866; this syndrome became well known as Down's Syndrome (Down, 1886). The association between matemal age and Down's Syndrome was first reported by Shuttleworth in 1909 (Shuttleworth, 1909). It was not until 1959 that the origin of Down's Syndrome was explained by the presence of an extra chromosome 21 (Lejeune et al., 1959) (Figure 1).

In 1966, the first successful chromosome analysis of human amniotic cells was reported (Steele and Breg, Jr., 1966) and soon after this, the first prenatal diagnosis of fetal Down's Syndrome was made (Valenti et al, 1968). In the seventies, maternal age as a screening parameter for fetal trisomy 21 was gradually introduced into clinical practice: in Belgium pregnant women $\geq 35$ years of age were offered amniocentesis, whereas many other countries used $\geq$ 37 years as cut off value. This screening method allowed an identification of approximately $30 \%$ of Down's Syndrome affected pregnancies (Abramowicz et al., 1998).

In 1984, Merkatz et al. reported the association between fetal aneuploidy and low maternal serum concentrations of Alfa-Foeto Protein (AFP) (Merkatz et al., 1984). Next, an association was reported between fetal Down's Syndrome and high maternal serum concentrations of Human Chorionic Gonadotropin (HCG) in 1987 (Bogart et al., 1987), and low concentrations of estriol ( $\left.\mathrm{E}_{3}\right)$ in 1988 (Wald et al., 1988a). These 3 parameters were combined with maternal age into the so-called "Triple test", which allowed to calculate an indivicual's risk for fetal Down's Syndrome more accurately than ever before (Wald et al., 1988b): approximately $60 \%$ of affected pregnancies could be identified at a false positive rate of $5 \%$. This test was introduced into Belgian obstetric care in 1992, when the first reports on the results of triple screening in routine practice were published (Wald et al., 1992). A further search for more maternal serum parameters revealed an association of fetal trisomy 21 with high concentrations 
Figure 1.1. Picture of a boy with Down's Syndrome or Trisony 21 (upper), an example of a human karyogran with three chromosomes 21 (lower left) and an example of three chromosomes 21, demonstrated by Fluorescent In Situ Hybridisation (FISH)( lower right).
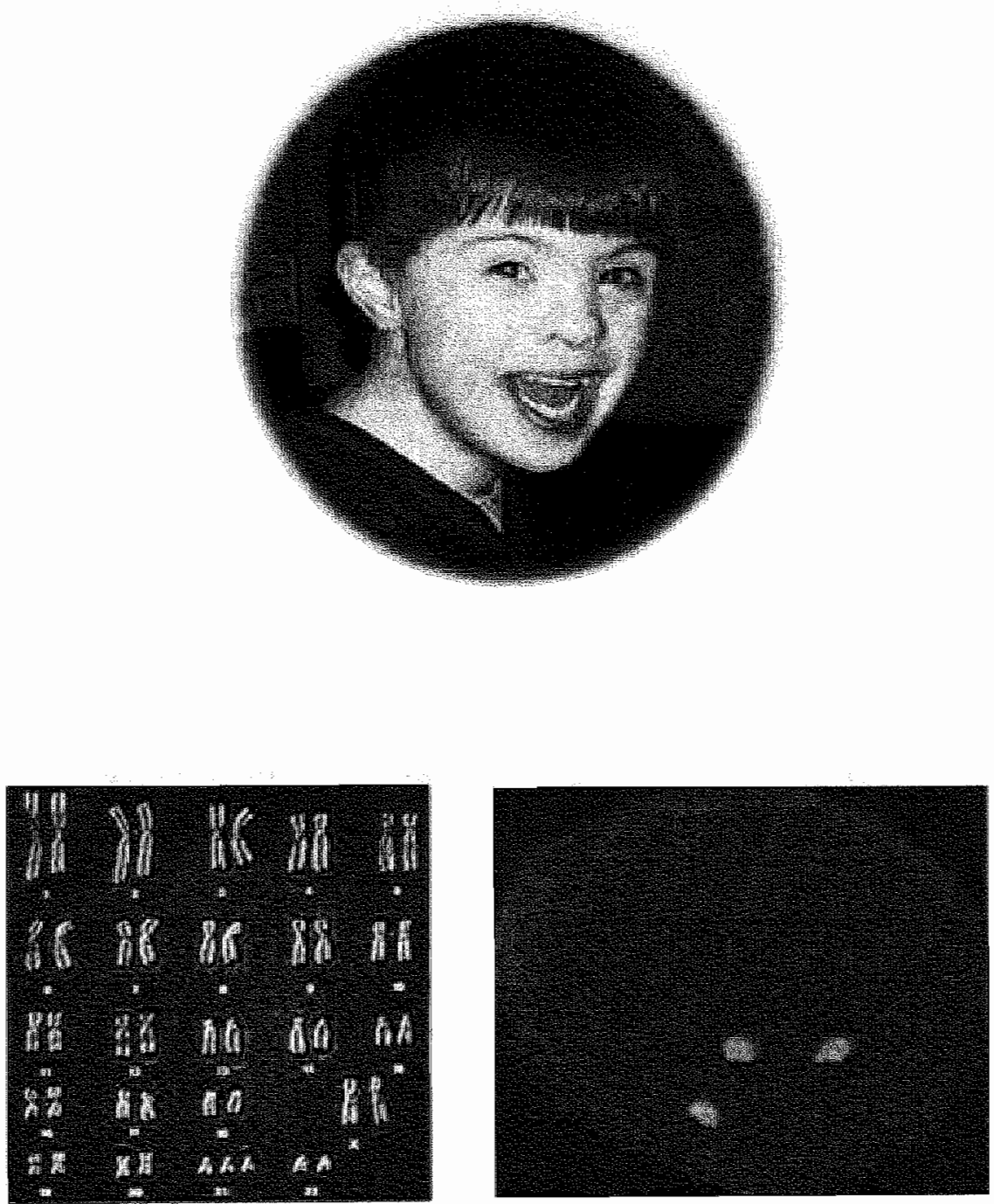

of immunoreactive Inhibin (van Lith et al., 1992) or dimeric Inhibin A (Cuckle et al., 1995) in the second trimester, low concentrations of Pregnancy Associated Plasma Protein A (PAPP-A) (Brambati et al, 1993) and high 
concentrations of free $\beta$ - HCG (Spencer et al., 1992) in the first trimester. This allowed for initiating the fetal aneuploidy screening process already in the first trimester, and for combinations with the triple test parameters in the so-called second trimester quadruple test (Wald et al., 1996a; Wald et al., 2003a) or the integrated test (Wald et al., 1999b; Wald et al., 2003b). This resulted in an important improvement of the overall screening performance.

Ultrasound was introduced in maternal serum screening algorithms for the adjustment of gestational age on the basis of fetal crown-rump length (CRL), which significantly improved the screening performance (Wald et al., 1994). Before that however, it was already reported that specific ultrasound markers were also associated with fetal chromosomal abnormalities. In 1985 Benaceraff et al reported the presence of nuchal edema in the neck of aneuploid fetuses in the second trimester (Benacerraf et al., 1985) and in 1990, nuchal translucency (NT) was recognised in the first trimester (Szabo and Gellen, 1990). An illustration of this NT is shown in Figure 2. The first report on the potential of this parameter in fetal aneuploidy screening was published in 1992 (Nicolaides et al., 1992). At the same time, it was reported that many of the fetal morphologic malformations, associated with chromosomal abnormalities, were recognisable by ultrasound (Nicolaides et al., 1993). As the association between chromosomal and structural abnormalities was not very consistent, it soon became clear that population screening for chromosomal abnormalities, based on structural malformations, would only result in a low detection rate (Abramowicz et al., 1998). The extended ultrasonic evaluation of fetal morphology in the second trimester, the so-called genetic scan, was evaluated extensively and reviewed recently by Bromley and Benaceraff (Bromley and Benacerraf, 2003). It is now mainly considered an option in addition to prior screening by maternal serum or ultrasound (Nyberg and Souter, 2003; Yeo and Vintzileos, 2003), however false negative end results have been reported. In the search for the identification of fetal aneuploidy by ultrasound, many other markers were linked to fetal Down's Syndrome, but none of these seemed to 
have the same discriminative power as NT (Nicolaides, 2003). Of all markers, the evaluation of the absence or presence of the fetal nasal bone (NB) in addition to NT seems to offer the most promising results (Nicolaides, 2003).

Figure 1.2. Example of fetal nuchat translucency thickness and fetal nasal bone, ats visualised by ultrasound.

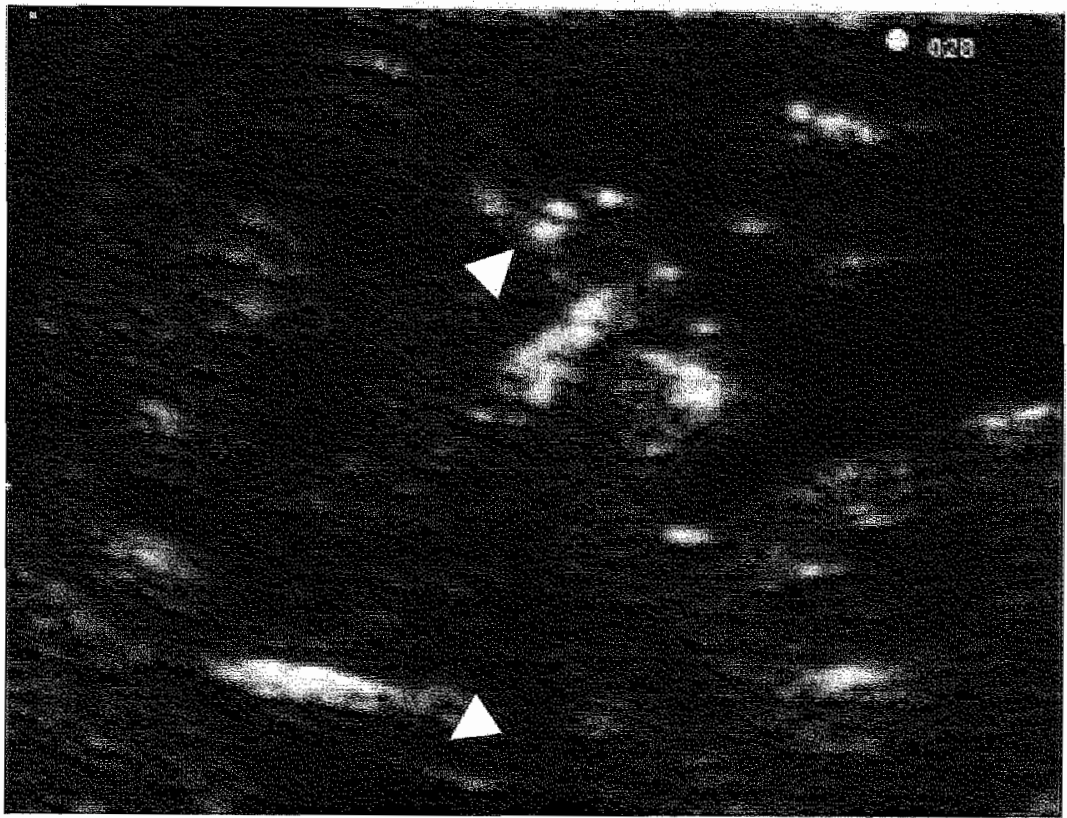

As the associations of maternal serum parameters and ultrasound markers with fetal chromosomal abnormalities were reported to be independent (Brizot et al., 1994), these parameters were soon combined into fetal aneuploidy screening algorithms, both in the first (Orlandi et al., 1997) and in the second trimester (Michailidis et al., 2001). The performance of these combinations was better than the performance of either method alone (Nicolaides, 2003) and comparable 
to the results of the second trimester quadruple test (Wald et al., 2003a). Based on a previous report on the integration of first and second trimester parameters (Wald et al., 1999b), a large scale study was conducted to examine the relevance of integrating as much relevant parameters as possible into one single algorithm. The results were published in 2003 (Wald et al., 2003b) and showed $\geq 90 \%$ detection rates for fetal Down's Syndrome at false positive rates of less than $5 \%$ : this was not only the best result ever reported, it also appeared to be the best cost-effective screening method.

The rapid evolution in the field of fetal aneuploidy screening and the development of many different parameters has caused a lot of confusion about the best choice to make for screening purposes (Goldberg, 2004). At present, consensus is growing that the best option for fetal aneuploidy screening today is the one which offers individualised perspectives to every pregnant woman, which are balanced between psychological, emotional, ethical and religious factors next to medical and economical arguments (Hewison, 2004).

\subsection{Comparison of different screening methods.}

Table 1 reviews the performance of the most important fetal aneuploidy screening methods currently used. These screening methods have been reviewed extensivelly in literature (Abramowicz et al., 1998; Nicolaides, 2003; Wald et al., 1997; Wald et al., 2003b). As is shown, the detection rate (DR) for fetal trisomy 21 (T21) at a fixed 5\% false positive rate (FPR) increases with the number of parameters used. In fact, all screening methods are based on one or two major types of parameters: maternal serum parameters and/or ultrasound parameters. The application of maternal serum screening and ultrasound screening in clinical practice is totally different; their intrinsic characteristics are enlisted in Table 2.

Sampling of maternal serum screening is very simple and as such, access to this screening method can be offered to most pregnant women in organised antenatal care. Many factors, such as multiple gestation, diabetes, maternal 
weight, artificial reproductive technologies and others, are known to interfere with the final screening results: in the algorithms for risk calculation, mathematical corrections are often required to adjust for these variables (Wald et al., 1997). A significant improvement of screening performance was observed after the implementation of ultrasound adjusted dates of conception into the maternal serum screening algorithms (Wald et al., 1997).

Table 1.1. Performance of different screening methods, as reponted in literature (Abramowicz et al, 1998; Nicolaides, 2003; Wald et al, 2003b). For every method, the detection rate for fetal trisomy 21 (DR) is expressed at a fixed $5 \%$ false positive rate (FPR).

Method

MA

$\mathrm{MA}+2 \mathrm{MSS}_{3}$

$\mathrm{MA}+1 \mathrm{MSS}$

$\mathrm{MA}+\mathrm{NT}$

$\mathrm{MA}+2 \mathrm{MSS}_{4}$

$\mathrm{MA}+\mathrm{NT}+1 \mathrm{MSS}$

$\mathrm{MA}+\mathrm{NT}+2 \mathrm{MSS}_{2}$

$\mathrm{MA}+\mathrm{NT}+\mathrm{NB}$

$\mathrm{MA}+1 \mathrm{MSS}+2 \mathrm{MSS}_{4}$

$\mathrm{MA}+\mathrm{NT}+\mathrm{NB}+1 \mathrm{MSS}$

$\geq 90$

$\mathrm{MA}+\mathrm{NT}+1 \mathrm{MSS}+2 \mathrm{MSS}_{4}$
DR (\%) at 5\% FPR

$30-40$

$60-70$

$60-70$

$60-80$

$70-80$

$80-90$

$80-90$

$80-90$

$80-90$

MA: maternal age

NT: nuchal transllucency

NB: nasal bone

IMSS: first urimester maternal serum screening

$2 \mathrm{MSS}_{\mathrm{x}}$ : second trimester maternall serum screening $(\mathrm{x}=$ the number of patameters used) 
The most important effect, caused by the introduction of NT-based screening method into clinical practice, was the move from screening in the second trimester to the first trimester (Cuckle, 2001). Although first trimester maternal serum screening was already known as an entity (Wald et al., 1996b), first trimester screening only became popular as a combination of maternal serum and ultrasound (Orlandi et al., 1997; Wald and Hackshaw, 1997).

Table 1.2. Characteristics of ultrasound screening and matemal serum screening in clinical practice.

\section{Screening by ultrasound}

Sampling of the most important parameters in the $1 \mathrm{st}$ trimester enhances

- 1st trimester diagnosis

- 1 st trimester termination of pregnancy

One day approach possible in set up of OSCAR - clinic

Fetus-specific screening results (twins)

Sampling is technically difficult: - guidelines

- training and audit

- time consuming examinations

Poor access for the total pregnant population in a limited number of expert centres

\section{Screening by maternal serum parameters}

Sampling is easy

Easy access for most pregnant women in routine prenatal care

Parameter handling and risk calculations in absence of patient

Pregnancy- (not fetus) specific

Many interfering factors, for which correction is necessary

Ultrasound dating is still necessary for optimal performance (dating)

For this combination, a one day approach of parameter sampling, risk calculation and invasive testing was introduced under the name "OSCAR- 
clinic" (One Stop Clinic for Assessment of Risk) (Nicolaides et al, 2002; Spencer et al., 2000). With this policy, it became possible to diagnose and terminate fetal aneuploidy- affected pregnancies at earlier stages than ever before. In contrast to maternal serum screening, ultrasound screening allows for fetus-specific evaluations in multiple pregnancies (Sebire et al., 1996). Ultrasound examinations are only possible in the presence of the pregnant woman and the whole process of parameter sampling is mostly visible to her on screen. As a result, difficulties to obtain appropriate measurements or closer evaluations of apparent abnormalities, may sometimes cause patient's' anxiety and stress, similar to high-risk scanning (Ayers and Pickering, 1997), even before the final screening result can be presented.

One of the major difficulties reported for first trimester ul.trasound screening is the need for accuracy of NT measurement in order to obtain valuable and consistent screening results, especially when no other parameters are used in the screening. A small deviation of the measured value may sometimes cause a calculated risk well above or below the cut off value for positive screening: an example of this is shown in Table 3.

Table 1.3. Influence of a deviation of $\leq 0.5 \mathrm{~mm}$ in the measured value of nuchal translucency on the final screening result of a 27 -year old womarn at a gestation of $11+4$ weeks (Crown-rump length $=50 \mathrm{~mm}$ ), as calculated by the screening algorithm distributed by the Fetal Medicine Foundation in 2000. 
Lack of methodological standardisation in NT measurement was the reason why the initial publications on NT-based screening reported detection rates for fetal trisomy 21 varying between $29 \%$ and $91 \%$ (Mallone et al., 2000). Guidelines towards standardisation of technique were then published (Nicolaides et al., 1999). It was suggested that training should be mandatory to obtain high quality and reproducible results (Braithwaite et al., 1996). An improvement of the overall screening performance after appropriate training was reported (Monni et al., 1997). To tackle the problem of technical inconsistency, a program of training courses on ultrasound screening for fetal aneuploidy, certification of competence and subsequent audit of performance was introduced by the Fetal Medicine Foundation (FMF)(Nicolaides et al., 1999;Snijders et al., 2002). An example of the impact of training on the distribution of measured NT-values in comparison to the FMF-reference range is shown in Figure 3.

Figure 1.3. Illustration of the impact of training and audit on ultrasonic measurement of NT. The solid line shows the distribution of the first 100 NT measurements by one ultrasonographer, which shows values well below the Fetal Medicine Foundation (FMF) reference range (black triangles). After audit and correction of technique according to the FMF guidelines, the distribution of a new sample of 100 measumements (intenupted line) is close to the FMF reference values.

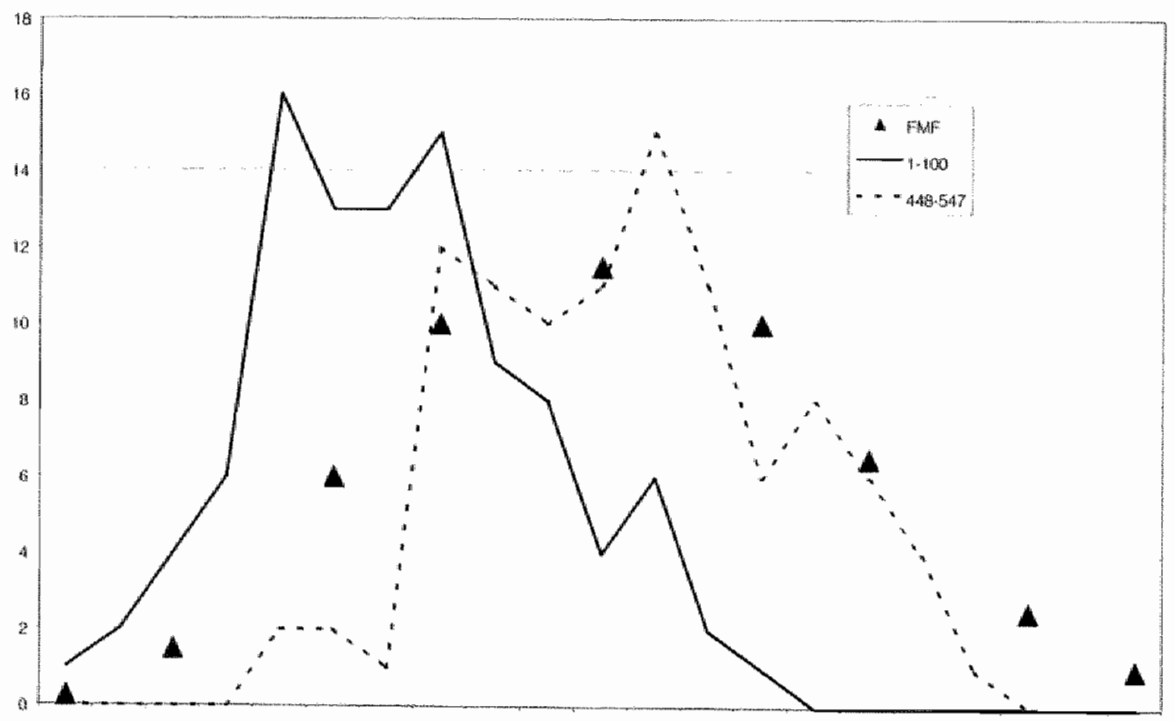


Major difficulties were encountered in introducing these highly specific ultrasound examinations into population screening for fetall aneuploidy (Benn, 2002). This caused a centralisation of NT-based screening in specialised, tertiary centres in the United States (Egan et al., 2002) and limitation of access for pregnant women to appropriately performed NT-measurements in the United Kingdom (Whittle, 2001). The introduction of high quality screening methods, which are easily accessible to the total population of pregnant women, is one of the most exciting challenges for health care organisations in many countries today.

\subsection{Screening at advanced maternal age.}

As discussed above, advanced maternal age was the first parameter used to screen populations for fetal aneuploidy. This screening method was introduced following the observation of an association between the prevalence of fetal trisomies and maternal age. Primary invasive testing, such as amniocentesis or chorionic villus sampling, was offered to women older than a cut off level of age or over the population $95^{\text {th }}$ percentile of maternal age. This method offered a 30-40\% detection of the total number of fetal trisomy (Abramowicz et al., 1998).

An association between detection rate and false positive rate of second trimester maternal serum screening and maternal age was reported in 1993 by Reynolds et al.(Reynolds et $a l$., 1993): the performance of this screening method was significantly better for women at advanced maternal age than for younger women. Soon after this in 1994, Haddow et al. reported a $75 \%$ reduction of amniocentesis for women $\geq 35$ years of age following maternal serum screening, meanwhile maintaining a trisomy 21 detection rate of $89 \%$ in this group (Haddow et al, 1994). This finding was confirmed in a population with a large proportion of women $\geq 35$ years (Shohat et al., 1995). Similar results were reported for ultrasound screening methods in 1995 (Szabo et al., 1995). The 
combination of both ultrasound and maternal serum screening methods was found to be the best option towards screening the advanced maternal age group, both in terms of performance (Dommergues et al., 2001;Egan et al., 2001; Rosen et al., 2002; Spencer, 2001) and cost-effectiveness (Hartnett et al., 2003).

In Flanders, Belgium, as in many other countries, mean maternal age increased during the last decades. According to the data of the "Studiecentrum voor Perinatale Epidemiologie" in Brussels, mean maternal age increased from 28.2 \pm 4.2 years in 1992 to $29.3 \pm 4.7$ years in 2001 , when $11.4 \%$ of pregnant women were aged $\geq 35$ years.

The steady increase of maternal age, the rather poor overall detection rate of fetal trisomy 21 by a policy of primary invasive testing for advanced maternal age and the availability of efficient and cost-effective screening methods for the total population, urged to rethink the practice of fetal aneuploidy screening in many countries and in Flanders, Belgium.

\subsection{Comparison between amniocentesis and chorionic villus sampling.}

Laboratory culture of fetal or placental cells is necessary for the prenatal diagnosis of genetic diseases. These cells are derived by ultrasound guided needle insertion and aspiration of amniotic fluid (amniocentesis) or placental tissue (chorionic villus sampling). As early amniocentesis (AC) is reported to be associated with fetal limb deformities, this procedure is not considered to be safe before 15 weeks of gestation (1998), whereas chorionic villus sampling (CVS) can be performed safely from as early as 10 weeks (Ammala et al., 1993). The invasive character of both tests implies a procedure related risk for pregnancy loss estimated around 0.5-1\% (Tabor et al., 1986). Differences between $\mathrm{AC}$ and CVS in procedure related complications were examined in four different studies and no significant findings were reported (1989; Ammala et al., 1993; Nicolaides ef al., 1994;Smidt-Jensen et al., 1992). Consequently, 
the way was cleared towards chorionic villus sampling as the invasive procedure of choice, in order to maintain the advantage of early termination of pregnancy in first trimester screening for fetal aneuploidy (Nicolaides et al., 1999). This view was supported by publications on medical, psychological and economical advantages of first trimester screening over second trimester screening (Caughey et al., 2002; Cuckle, 2001; Cusick et al., 2003; de Graaf et al., 2002). A recent Cochrane meta-analysis however, reported a 1.33 odds ratio for complications of CVS over AC (Alfirevic et al., 2003). These complications were both technical (failure of sampling, placental mozaicism, laboratory handling) and clinical (loss of pregnancy). These data clearly illustrate the need for reflection on the balance between advantages and disadvantages of first trimester diagnostic tests for fetal aneuploidy screening. This problem can only be solved by appropriate and unbiased patient counselling, allowing an individualised approach for every pregnant woman faced with the option of fetal diagnosis.

\subsection{Economic aspects.}

Several studies report on the aspects of economic costs and benefits of fetal aneuploidy screening (Abramowicz et al., 1998; Rozenberg et al., 2002; Shackley et al., 1993; Torrance, 1986; Wald et al., 1997; Wald et al., 2003b). The conclusions of these reports are very similar: fetal aneuploidy screening may cause an important decrease of public health care expenses. Screening methods using maternal serum parameters are considered the most expensive strategies, however balanced against the public costs of undetected chromosomal abnormalities these strategies are still economical beneficial (Gilbert et al., 2001). Until now, no study reported an economic loss caused by one of these strategies, and even a policy of offering invasive testing to all pregnant women was considered cost-effective (Harris et al., 2004).

A recent study compared costs and benefits between several screening strategies: the combinations of first trimester ultrasound and first or second 
trimester maternal serum parameters were considered the most effective options, and clearly more superior to screening by maternal serum or maternal age only (Gilbert et al., 2001). A modelled calculation in the SURUSS report showed that, from both medical and economical points of view, the most effective screening strategy was the one which integrates all first and second trimester parameters into one risk calculation (integrated screening) (Wald et al., 2003b).

It is important to consider that most studies on the economic aspects of medical care are modelled studies, based on the assumption that population screening is performed by universal application of one single strategy in comparison to the universal application of others. In reality, these situations are rarely true: in many populations a mixture is found of different fetal aneuploidy screening methods (Egan et al., 2002; Wald et al., 1999a). Sometimes, these different screening methods may even be performed sequentially in the same pregnancy (Maymon et al., 2001;Schuchter et al., 2001). In Belgium today, there are no general guidelines on fetal aneuploidy screening. This is reflected in the variety of indications for diagnostic amniocentesis, as published by one of the centres for human genetics (Witters, 2002). It is totally unclear how this variety of strategies influences the total costs of screening, but it is very likely that a structured and well organised public screening program would be beneficial from both the medicall and the economical point of view.

\subsection{Ethical aspects and patient's counselling.}

In current antenatal care, fetal aneuploidy screening has become one of the most important issues related to the basic principles of medical ethics: autonomy, beneficence and maleficence (Chasen et al., 2001). The personal choice of every couple is to be respected, whatever nationality, religion, social class and race. It is wrong to force someone to have a screening test; it is equally wrong to deny a screening to someone who may benefit from it. Professionals in antenatal care should inform every pregnant woman about the possibilities and 
limitations of fetal aneuploidy screening tests, and to allow them to make a free choice. In order to transmit accurate and up to date information, it is important that these professionals keep up with the current evolutions in the field, even when these occur very fast as in the last decade. It is also very important to start this communication at the very first chance, perhaps even before conception. The information should be available in written form or leaflets: this allows couples to consider and reconsider the different options before the first parameter is sampled. Every screening always includes the possibility to start a cascade of tests and interventions, which often goes with patient's anxiety and emotional stress. This information should be neutral and non-directive, in order to allow every couple to choose independently from external or circumstantial factors. To help with this, training in communication techniques and/or the support of professional medical counsellors may be very useful.

\subsection{Promising developments for the near future.}

Currently, several publications report promising results on technicues to isollate fetal cells or fetal DNA from maternal blood (Holzgreve, 1997). This technique should allow performing specific genetic tests on fetal cells without the need for invasive procedures and bypassing the procedure related risk of pregnancy loss. In view of the economic benefits expected from offering diagnostic procedures to all pregnant women (Harris et al., 2004), this technique has the potential to become a standard procedure in future. However, many practical problem are still to be resolved before this screening method can be introduced into daily clinical practice (Holzgreve, 1997). Today, highly sensitive and specific screening tests are still needed to distinguish between low risk and high risk, in order to enable a rational, cost-beneficial and individualised high quality care for the whole pregnant population. In this perspective, the search for more and new screening parameters and measures towards optimisation of current screening practice is to continue. 


\subsection{Population screening in Belgium and in the Netherlands.}

The organisation of public health care is different in Belgium and the Netherlands. This difference is also reflected in the fetal aneuploidy screening activities in both countries.

In Belgium, public health care is organised as a private service. Medical activities are reimbursed equally to all citizens in a National Health Insurance System. Hospitals, health care workers and medical institutions are funded or paid per fee. In 2002, the Belgian Patient's Charter was published (Belgisch Staatsblad 26/9/2002). This law declares the primary legal. rights for every patient, such as the right to choose a doctor, an investigation or a treatment and the right for information on medical activities. In the Belgian system, there is a place for private initiatives towards development and provision of high quality medical care. This is illustrated by the Medline enlisted publications from Centres for Obstetrics and Gynaecology in Flanders: 17\% (23/136) of the reports in 2003 were from non-academic centres (Wetenschappelijke Raad van de VVOG, 2004). However, the difference between levels of primary and medium care and between levels of medium and high care may not always be clear. National guidelines on public health care services are poor and systems towards audit and quality control of medical care are only just starting. In Belgium today, a variety of fetal aneuploidy screening services is available to all pregnant women (see Chapters 2 and 10).

In the Netherlands, there is also a National Health Insurance System, but this is not equal to all citizens. Medical health care is structured in levels of primary, medium and high care and there are many guidelines and regulations to control the provision of services. Exceptions to these regulations are only possible at patient's expenses. In order to prevent possible insults to mental or physical health, law restricts population studies and investigations, such as screening for fetal aneuploidy (Wet Bijzondere Medische Verrichtingen, Art 2: 
"Vergunningsplicht"). For many years now, fetal aneuploidy screening in the Netherlands is available through offering screening tests and/or diagnostic invasive procedures (AC or CVS) to women aged 36 years or more (Nagel et al., 2004). In 2001, the Health Council of the Netherlands ("Gezondheidsraad") requested the National Government to ensure the provision of fetal aneuploidy screening tests to all pregnant women (Gezondheidsraad, 2001). This was refused : the intention to interfere medically with the natural course of every pregnancy and the possible arousal of a public desire for the illusive perfect child, were considered immoral. Today, it is lawfully recommended to inform all pregnant women about the options for fetal aneuploidy screening ("informeren") but to offer the tests only to women $\geq 36$ years ("aanbieden"). From the medical and ethical point of view, this approach however was considered controversial and ambiguous (de Wert, 2004). The Government's insinuations into the integrity of medical experts, involved in the development and provision of the screening tests, were also contested fiercely (Sheldon, 2004). All the same, the discussion on the organisation of a national screening program and the limitation of local or private initiatives is ongoing for many years now (van Schielen et al., 2004). Recently, it was proposed to organise screening programs within regions (Veersema et al., 2005). For the time being, fetal aneuploidy screening in the Netherlands is not (yet) available to all pregnant women.

References

(1989) Multicentre randomised clinical trial of chorion villus sampling and amniocentesis. First report. Canadian Collaborative CVS-Amniocentesis Clinical Trial Group. Lancet 1: 1-6.

(1998) Randomised trial to assess safety and fetal outcome of early and midtrimester amniocentesis. The Canadian Early and Mid-trimester Amniocentesis Trial (CEMAT) Group. Lancer $351: 242-247$. 
Abramowicz,M.., Reynolds, T., Varnos, $E_{, 3}$ and Zimmermann,R. (1998) Screening for chromosime and genetic abnormalities. In Textbook of perinatal medicine: a comprehersine guide to modem clinical perinatology. Kurjac, $A$. (ed.) London; New York: Parthenon Publishing Group Ltd, pip. 610-637.

Alfirevic, Z. Gosden, C. M. and Neilson, J. (2003) Chorion villus sampling wersus amniocentesis for prenatall diagnosis (Cochrane review). Cochrane Library 2003.

Ammala, P., Hiilesmaa, V. K., Liukkonen, S., Saisto, T., Teramo, K., and von Koskull, H. (1993) Randomized trial comparing first-trimester transcervical chorionic villus sampling and secondtrimester amniocentesis. Prenat Diagn 13:919-927.

Ayers, S. and Pickering, A. D. (1997) Psychological factors and ultrasound: differences between routine and high-risk scans. Ultrasound Obstet Gynecol 9: 76-79.

Benacerraf, B. R., Barss, V. A., and Laboda, L. A. (15-4-1985) A sonographic sign for the detection in the second trimester of the fetus with Down's syndrome. Am $J$ Obstet Gynecol 151 : 1078-1079.

Benn, P. A. (2002) Advances in prenatal screening for Down syndrome: Ill first trimester testing, integrated testing, and future directions. Chin Chim Acta 324: 1-11.

Bogart, M. H., Pandian, M. R., and Jones, O. W. (1987) Abnormal maternal serum chorionic gonadotropin levels in pregnancies with fetal chromosome abnomalities. Prenat Diagn 7: 623630.

Braithwaite, J. M., Kadir, R. A., Pepera, T. A., Morris, R. W., Thompson, P. J., and Economides, D. L. (1996) Nuchal translucency measurement: training of potential examiners. Uttrasound Obstet Gynecol 8: 192-195.

Brambati, B., Macintosh, M. C., Teisner, B., Maguiness, S., Shrimanker, K., Lanzani, A. Bonacchi, I., Tului, L.. Chard, T., and Grudzinskas, J. G. (1993) Low matenal serum levels of pregnancy associated plasma protein A (PAPP-A) in the first trimester in association with abnormal fetal karyotype. Br J Obster Gynaecol 100:324-326.

Brizot, M. L., Sniders, R. J., Bersinger, N. A., Kuhn, P., and Nicolaides, K. H. (1994) Maternal serum pregnancy-associated plasma protein $A$ and fetal nuchal translucency thickness for the prediction of fetal trisomies in aarly pregnancy. Obstet Gynecol 84: $918-922$.

Bromley, B. and Benacerral, B. R. (2003) The Genetic Sonogram Scoring Index. Semin Perinarol 27: $124-129$

Caughey, A. B., Kuppermann, M.. Norton, M. E., and Washington, A. E. (2002) Nuchal translucency and first trimester biochemical markers for down syndrome screening: a costeffectiveness analysis. Am J Obster Gynecol 187: 1239-1245.

Chasen, S. T." Skupski, D. W., McCullough, L. B., and Chervenak, F. A. (2001) Prenatal informed consent for sonogram: the time for first-trimester nuchal translucency has come. $J$ Ultrasound Med 20: 1147-1152.

Cuckle, H. (17-11-2001) Time for total shift to first-trimester screening for Down's syndrome. Lancet 358 : $1658-1659$. 
Cuckle, H. S., Holding, S., Jones, R., Wallace, E. M., and Groome, N. P. (1995) Maternal serm dimeric inhibin A in second-trimester Down's syndrome pregnaneles. Prenat Diagris 15:385-386.

Cusick, W., Buchanan, P., Hallahan, T. W., Krantz, D. A., Larsen, J. W., Jr, and Macr, J. N. (2003) Combined frst-trimester versus second-trinester serum sereening for Down syathome: A cost analysis. Am J Obster Gynecol 188: 745-751.

de Graaf, I. M., Tijmstra, T., Bleker, O. P., and van Lith. J. M. (2002) Womens' preference in Down syadrome screening. Prenat Diagn 22: 624-629.

de Wert, G. (2004) Een bom onder de wet. Medisch Contact 59: 212-214.

Dommergues, M., Audibert, F., Benattar, C., Champagne, C., Gomel, V., and Frydman, R. (2001) Is routine ammiocentesis for advanced maternal age still indicated? Fetal Diagn Ther 16:372-377.

Down, L. (1886) Observations on an ethnic classification of idiots. Clin Lectures and Reports, London Hospital 3: 259-262.

Egan, J. F., Kaminsky, L. M., DeRoche, M. E., Barsoom, M. J., Borgida, A. F., and Bemn, P. A. (2002) Antenatal Down syndrome screening in the United States in 2001 : a survey of maternalfetal medicine specialists. Am J Obstet Gynecol 187: 1230-1234.

Egan, J. F., Malakh, L., Turner, G. W., Markenson, G., Wax, J. R., and Bem, P. A. (200l) Role of ultrasound for Down syndrome screening in advanced maternal age. Am J Obstet Oynecol 185 : 1028-1031.

Gezondheidsraad (2001) Prenatale screening: Downsyndroom, neuralebuisdefecten, routineechoscopie. Den Haag.

Gilbert, R. E., Augood, C., Gupta, R., Ades, A. E., Logan, S., Sculpher, M., and Der Meulen, J. H. (25-8-2001) Screening for Down's syndrome: effects, safety, and cost effectiveness of first and second trimester strattegies. BMJ 323: 423-425.

Goldberg, J. D. (2004) Down's Syndrome screening: confusion or consensus? BJOG 111:517518.

Haddow, J. E., Palomaki, G. E., Knight, G. J., Cunningham, G. C., Lustig, L. S., and Boyd, P. A. (21-4-1994) Reducing the need for amniocentesis in women 35 years of age or older with serum markers for screening. N Engl J Med 330: 1114-1118.

Harris, R. A., Washington, A. E. Nease, R. F. Jr, and Kuppermann, M. (24-1-2004) Cost utility of prenatal diagnosis and the risk-based threshold. Lancet $363: 276-282$.

Hartnett, J., Borgida, A. F., Benn, P. A., Feldman, D. M., DeRoche, M. E., and Egan, J. F. (2003) Cost analysis of Down syndrome screening in advanced matemal age. J Matern Fetal Neonatal Med 13: 80-84.

Hewison, 1. (2004), Psychological aspects of integrated testing. BJOG I I I: 519.520 .

Holzgreve, W. (1997) Will ultrasound-screening and ultrasound-guided procedures be replaced by non-invasive techniques for the diagnosis of fetall chromosome anomalies? Uirasound Obstet Gynecol 9: 217-219. 
Lejeune, J., Gautier, M., and Turpin, R. (16-3-1959) [Study of somatic chromosomes from 9 mongoloid children.]. C R Hebd Seances Acad Sci 248: 1721-1722.

Malone, F. D., Berkowitz, R. L., Canick, J. A., and D'Alton, M. E. (2000) First-lrimester screening for aneuploidy: research or standard of care? Am J Obstet Gynecol 182: 490-496.

Maymon, R., Bergman, M., Segal, S., Dreazen, E., Weinraub, Z., and Heman, A. (2001) Sequential first and second trimester screening tests: correlation of the markers levels in normal versus Down syndrome affected pregnancies. Prenat Diagn 21:1175-1177.

Merkatz, J. R., Nitowsky, H. M., Macri, J. N., and Johnson, W. E. (1-4-1984) An association between low matermal serum alpha-fetoprotein and fetal chromosomal abnormalities. Am I Obstet Gynecol 148: 886-894.

Michailidis, G. D., Spencer, K., and Economides, D. L. (2001) The use of nuchal translucency measurement and second irimester biochemical markers in screening for Down's syndrome. B./OG 108: 1047-1052.

Monni, G., Zoppi, M. A., Ibba, R. M., and Floris, M. (29-11-1997) Fetal nuchal translucency test for Down's syndrome. Lancet 350: 1631- 1632 .

Nagel, H. T., Knegt, A. C., Kloosterman, M. D., Wildschul, H. I., Leschot, N. J., and Vandenbussche, F. P. (31-7-2004) [Inwasive prenatal diagnosis in the Netherlands, 1991-2000: number of procedures, indications and abnormal results detected]. Ned Tijdschr Geneeskd 148: $1538-1543$.

Nicolaides, K., Brizol, Mde L., Patel, F., and Snijders, R. (13-8-1994) Comparison of chorionic villus sampling and amniocentesis for fetal karyotyping at 10-13 weeks' gestation. Lancet 344: 435-439.

Nicolaides, K., Shawwa, L., Brizot, M., and Snijders, R. (1-1-1993) Ultrasonographically detectable markers of fetal chromosomal defects. Ulirasound Obsiet Gynecol 3: 56-69.

Nicolaides, K. H. (2003) Screening for chromosomal defects. Ultrasound Obstet Gynecol 21 : $313-321$.

Nicolaides, K. H. Azar "G., Byrne, D., Mansur, C., and Marks, K. (4-4-1992) Fetall nuchal translucency: ultrasound screening for chromosomal defects in first trimester of pregnancy. BM. 304: $867-869$.

Nicolaides, K. H., Bindra, R., Heath, V., and Cicero, S. (2002) One-stop clinic for assessment of risk of chromosomal defects at 12 weeks of gestation. J Marem Fetal Neonatal Med 12: 9-18.

Nicolaides,K.H., Sebire.NJ., and Snijders,R.J. (1999) Nuchal translucency and chromosomal defects. In The 11 - I4 week scan; the diagnosis of fetal abnomalities. Nicolades, K.H., Sebite,NJ.., and Snijders, R.J. (eds.) London; New York: Parthenon Publishing Group Lid, pp. 366.

Nyberg. D. A. and Souner, V. L. (2003) Use of genetic sonography for adjusting the risk for fetal Down syndrome. Semin Perinatal 27: 130- 144 . 
Orlandi, F., Damiani, G., Hallahan, T. W., Krantz, D. A, and Macri, J. N. (1997) First-trimester screening for fetal aneuploidy: biochemistry and nuchal translucency. Oltrosound Obstet Gynecol $10: 381-386$.

Reynolds, T. M., Nix, A. B., Dunstan, F. D., and Dawson, A. J. (1993) Age-specific detection and false-positive rates: an aid to counseling in Down syndrome risk sereening. Obstet Gynecol 81: $447-450$.

Rosen, D. I., Kedar, I., Amiel, A., Ben Tovim, T., Petel, Y., Kaneti, H., Tohar, M., and Fejgin, M. D. (2002) A negative second trimester triple test and absence of specific ultrasonographic markers may decrease the need for genetic amniocentesis in advanced maternal age by $60 \%$. Prenat Diagh 22: 59-63.

Rozenberg, P., Malagrida, L., Cuckle, H., Durand-Zaleski, L, Nisand, I., Audibert, F., Benattar, C., Tribalat, S., Cartron, M., Lemarie, P., Stoessel, J., Capolagui, P., Janse-Marec, J., Barbier, D., Allouch, C., Perdu, M., Roberto, A., Lahna, Z., Giudicelli, Y., and Ville, Y. (2002) Down's syndrome screening with muchal translucency at $12(+0)-14(+0)$ weeks and maternal serum markers at 14(+1)-17(+0) weeks: a prospective study. Hum Reprod 17: 1093-1098.

Schuchter, K., Hafner, E., Stangl, G., Ogris, E., and Philipp, K. (2001) Sequential screening for trisomy 21 by nuchal translucency measurement in the first trimester and maternal serum biochemistry in the second trimester in a low-risk population. Ultrasound Obstet Gynecol 18: 23 25.

Sebire, N. J., Snijders, R. J., Hughes, K., Sepulveda, W., and Nicolaides, K. H. (1996) Screening for trisomy 21 in twin pregnancies by maternal age and fetal nuchal translucency thickness at $10-$ 14 weeks of gestation. Br J Obstet Gynaecol 103: 999-1003.

Shackley, P., McGuire, A., Boyd, P. A., Dennis, J., Fitchett, M., Kay, J., Roche, M., and Wood, P. (1993) An economic appraisal of alternative pre-natal screening programmes for Down's syndrome. J Public Health Med 15: 175-184.

Sheldon, T. (19-6-2004) Dutch minister questions integrity of health advisers. BMJ 328: 1456.

Shohat, M., Legum, C., Romem, Y., Borochowitz, Z., Bach, G., and Goldman, B. (1995) Down syndrome prevention program in a population with an older maternal age. Obstet Gynecol 85: 368-373.

Shutileworth, G. (1909) Mongolian imbecility. BMJ 2: 661-665.

Smidt-Jensen, S., Permin, M.» Philip, J., Lundsteen, C., Zachary, J. M., Fowler, S. E., and Gruning, K. (21-11-1992) Randomised comparison of amniocentesis and tratusabdominal und transcervical chorionic villus sampling. Lancet 340: 1237-1244.

Snijders, R. J., Thom, E. A., Zachary, J. M., Platt, L. D., Greene, N., Jackson, L. G., Sabbagha, R. E., Filkins, K., Silver, R. K., Hogge, W. A., Ginsberg, N. A., Beverly, S., Morgan, P., Blum, K., Chilis, P., Hill, L. M., Hecker, J, and Wapner, R. J. (2002) First-trimester trisomy screening: nuchal translucency measurement training and quality assurance to correct and unify technique. Ulrasound Obstet Gynecol 19: 353-359. 
Spencer, K. (2001) Age related detection and false positive rates when screening for Down's syndrome in the first trimester using fetal nuchal translucency and maternal serum free betahCG and PAPP A. BJOG 108: 1043-1046.

Spencer, K. Macri, J. N., Aitken, D. A., and Conmor, J. M. (13-6-1992) Free beta-hCG as firsttrimester marker for fetal trisomy. Lancer $339: 1480$.

Spencer, K., Spencer, C. E., Power, M., Moakes, A., and Nicolaides, K. H. (2000) One stop clinic for assessment of risk for fetal anomallies: a report of the first year of prospective screening for chromosonal anomalies in the first trimester. B.JOG 107: 1271-1275.

Stecle, M. W. and Breg, W. R., Jr. (19-2-1966) Chromosome analysis of human amniotic-fluid cells. Lancet I: 383-385.

Szabo, J. and Gellen, J. (3-11-1990) Nuchal fluid accumulation in trisomy-21 detected by vaginosonography in first trimester. Lancet 336:11.33.

Szabo, J., Gellen, J., and Szemere, G. (1995) First-trimester ultrasound screening for fetal aneuploidies in women over 35 and under 35 years of age. Ultrasound Obstet Gynecol 5: 161 163.

Tabor, A., Philip, J, Madsen, M., Bang, J., Obel, E. B., and Norgaard-Pedersen, B. (7-6-1986) Randomised controlled trial of genetic amniocentesis in 4606 low-risk women. Lancet 1: 1287 1293.

Torrance, G. W. (1986) Measurement of health state utilities for economic appraisal. $J$ Health Econ $5 ; 1-30$.

Valenti, C., Schutta, E. J., and Kehaty, T. (27-7 1968) Prenatal diagnosis of Down's syndrome. Lancet 2: 220 .

van Lith, J. M., Pratt, J. J., Beekhuis, J. R., and Mantingh, A. (1992) Second-trimester maternal serum immunoreactive inhibin as a marker for fetal Down's syndrome. Prenat Diagn 12: 801-806.

wan Schielen, P. C., van Lith, J. M., and Loeber, J. G. (2004) Dossier prenatale screening. Uniforme screening: testen op Downsyndroom niet gebaat bij vrij ondememerschap. Medisch Contact 59: 1516-1518.

Veersema, D., Die-Smulders, C. E. M., and Nijhuis, J. G. (2005) Alle zwangeren testen: prenatale screening op Downsyndroom regionaal opgezet. Medisch Contact 60: 356-358.

Wald, N. J., Cuckle, H. S., Densem, J. W., Nanchahal, K., Canick, J. A., Haddow, J. E., Knight, G. J., and Palomaki, G. E. (1988a) Maternal serum unconjugated oestriol as an antenatal screening test for Down's syndrome. Br J Obster Gynaecol 95: 334-341.

Wald, N. J., Cuckle, H. S., Densem, J. W., Nanchahal, K., Royston, P., Chard, T., Haddow, J. E, Knight. G. J" Palomaki, G. E., and Canick, J. A. (8-10-1988b) Maternal serum sereening for Down's syndrome in early pregnancy. BMI 297: 883-887.

Wald, N. J., Densem, J. W., George, L., Muttukrishna, S., and Knight, P. G. (1996a) Prenatal screening for Down's syndrome using inhibin-A as a serum marker. Prenat Diagn 16: 143-153. 
Wald, N. I., Densem, J. W., Smith, D., and Klee, G. G. (1994) Four-marker senm screening for Down's syndrome. Prenat Diagn 14: 707-716.

Wald, N. J., George, L., Smith, D., Densem, J. W., and Petterson, K. (1996b) Serum sereening for Down's syndrome between 8 and 14 weeks of pregnancy. International Prenatal Screening Research Group. Br J Obstet Gynaecol 103: 407-412.

Wald, N. J. and Hackshaw, A. K. (1997) Combining ultrasound and biochemistry in firsttrimester screening for Down's syndrome. Prenat Diagn 17: 821-829.

Wald, N. J., Huttly. W. J., and Hackshaw, A. K. (8-3-2003a) Antenatal screening for Down's syndrome with the quadruple test. Lancet $361: 835 * 836$.

Wald, N. J., Huttly, W. J., and Hennessy, C. F. (9-10-1999a) Down's syndrome screening in the UK in 1998. Lancet 354: 1264.

Wald., N. J., Kennard, A., Densem, J. W., Cuckle, H. S., Chard, T., and Butler, L. (15-8-1992) Antenatal maternal serum screening for Down's syndrome: results of a demonstration project. BMJ 305: 391-394.

Wald, N. J., Kemnard, A., Hackshaw, A., and McGuire, A. (1997) Antenatal screening for Down's syndrome. J Med Screen 4: 181-246.

Wald, N. J., Rodeck, C., Hackshaw, A. K., Walters, J., Chitty, L., and Mackinson, A. M. (2003b) First and second trimester antenatal screening for Down's syndrome: the results of the Serum, Urine and Ultrasound Screening Study (SURUSS). J Med Screen 10; 56-104.

Wald, N. J., Watt, H. C., and Hackshaw, A. K. (12-8-1999b) Integrated screening for Down's syndrome on the basis of tests performed during the first and second trimesters. $N$ Engl J Med 341: $461-467$.

Wetenschappelijke Raad van de VVOG (2004) Internationale publicaties VVOG-leden 2003. Sint-Niklaas, Belgium.

Whittle, M. (2001) Down's syndrome screening: where to now? BJOG 108: 559-561.

Witters, I. Prenatal diagnosis of fetall malformations and chromosomal anomalies: a multidisciplinary approach. 7-158. 2002. Catholic University Louvain. 2002.

Yeo, L. and Vintzileos, A. M. (2003) The use of genetic sonography to reduce the need for amniocentesis in women at high-risk for Down syndrome. Semin Perinafol 27: 152-159. 
Chapter 2

\section{Fetal aneuploidy screening in}

\section{Belgium between 1995 and 2002} Aims of the study

Acknowledgement to

Dr. Raf Mertens, Christelijke Mutualiteit Brussels, for his contribution to this chapler. 


\section{Summary}

The proportion of pregnancies at advanced maternal age increased in Belgium between 1995 and 2002. In the same period, there was also an increase of maternal serum screening and fetal karyotyping. The $1.9 \%$ increase of fetal karyotyping in the total population registered by the health insurance company $\mathrm{CM}$, was mainly the result of more primary invasive testing at maternal age $\geq$ 35 years, and to a lesser extend due to more fetal karyotyping following maternal serum screening at $<35$ years. Maternal serum screening at $\geq 35$ years was associated with a decrease of fetal karyotyping. This controversial evolution was the onset of the study presented in this thesis. 


\subsection{Fetal aneuploidy screening in Belgium between 1995 and 2002 .}

In Belgium, there is no national registry on congenital malformations or prenatal screening activities. To study the practice of fetal aneuploidy screening in the country, information was obtained from different sources and databases, al $₫$ of which appeared to contain partial information on the subject. This limitation has to be considered carefully when interpreting the data presented in this chapter.

Screening identifies a proportion of pregnant women at risk for fetal aneuploidy, who are offered invasive diagnostic procedures such as amniocentesis or chorionic villus sampling. At the end of 2001, there were 5 different screening methods commonly practised in Belgium: (1) screening by maternal age only, (2) screening by maternal age and second trimester maternal serum parameters, (3) screening by maternal age and nuchal translucency (NT) measurement, (4) screening by maternal age, NT and second trimester maternal serum parameters and (5) screening by maternal age, NT and first trimester maternal serum parameters. Between August 2000 and January 2002, a total of 1405 diagnostic invasive procedures were performed in the Centre for Human Genetics in Louvain: of these, $492(35 \%)$ were for advanced maternal age, 391 (28\%) were for positive second trimester maternal serum screening, $106(7.5 \%)$ were for NT or other ultrasound detected soft markers, and the remaining were for indications different from screening.

In 2002, we studied the evolution of fetal aneuploidy screening activities in Belgium between 1995 and 2001. As the National Health Insurance System reimburses all medical procedures to Belgian citizens, we searched the database of the National Institute of Health Insurance (RIZIV) for the yearly number of pregnancies $\geq 12$ weeks, the number of fetal karyotyping (FK) and the number of maternal serum screening (MSS) tests. The data are shown in Table 1. 
Table 2.1. The number of pregnancies $\geq 12$ weeks, maternal serum screening tests and fetal karyotyping in the total Belgian population between 1995 and 2001 , as registered by the National Institute for Heaith Insurance (RIZIV).

\begin{tabular}{cccccccc} 
& 1995 & 1996 & 1997 & 1998 & 1999 & 2000 & 2001 \\
\hline Pregn >= 12W & 105815 & 110156 & 108279 & 107408 & 108685 & 110239 & 112616 \\
Mat serum tests & & & & & 17910 & 59230 & 62437 \\
$\%$ & & & & & 16.5 & 53.7 & 55.4 \\
Fetall karyo & 10759 & 11220 & 11893 & 10384 & 12570 & 11802 & 13226 \\
$\%$ & 10.2 & 10.2 & 11 & 9.7 & 11.6 & 10.7 & 11.7 \\
\hline
\end{tabular}

Over the time period studied, there was a $1.5 \%$ increase of FK. MSS was performed in $55 \%$ of pregnancies in 2001 . As RIZIV was unable to provide data on MSS before 1999 or to reproduce details in different subgroups of the population, we searched the database of Christelijke Mutualiteiten (CM) for the same parameters as described above. $\mathrm{CM}$ is the largest mutual health insurance fund in Belgium, covering $41-48 \%$ of all Belgian pregnancies between 1995 and 2001. For each item, the CM database was more detailed than the RIZIV register and allowed for a more comprehensive evaluation.

The results of this search are enlisted in Table 2. Compared to the RIZIV register, the MSS rate in the CM database is very similar in 2000 and 2001 , but the yearly FK rate is approximately $2 \%$ lower. Between 1995 and 2001 , there was an overall increase of the proportion of pregnancies at maternal age $\geq 35$ years and of the proportion of pregnancies submitted to MSS and/or to FK. The evolution of these medical activities however was totally different for women $\geq$ 35 years of age compared to younger women. In the advanced maternal age group, there was an increase of FK without prior MSS, with an estimated linear trend of $+4.5 \%$ over the period studied. Simultaneously, there was a $4.7 \%$ decrease of FK following MSS. Yet, the total rate of MSS and FK remained unchanged. In the younger maternal age group however, there was an increase 
Table 2.2. The number of pregnancies $\geq 12$ weeks ( $\mathrm{Pr} \geq 12 \mathrm{~W})$ and the estimated linear trends (ELT) of maternal serum screening tests (MSS) and fetal karyotyping (FK) in two matemal age growps between 1995 and 2001 , als registered by the mutual health insurance fund CM. The CM population is $41-48 \%$ of the total Belgian population (see Table 1).

$19951996 \quad 1997 \quad 1998 \quad 1999 \quad 2000 \quad 2001 \quad$ ELT $^{*} \quad$ P*

\begin{tabular}{ccccccccccc|}
\hline Tatal & & & & & & & & & & \\
Pr $>=12 \mathrm{~W}$ & 50791 & 51781 & 50061 & 49441 & 48502 & 47882 & 45877 & & \\
MSS & 21575 & 24644 & 24547 & 24109 & 25093 & 25799 & 25257 & & \\
$\%$ & 42.5 & 47.6 & 49 & 48.8 & 51.7 & 53.9 & 55.1 & $+11.3 \%$ & $<0.001$ \\
FK total & 3926 & 4329 & 4066 & 4234 & 4303 & 4601 & 4437 & & \\
$\%$ & 7.7 & 8.4 & 8.1 & 8.6 & 8.9 & 9.6 & 9.7 & $+1.9 \%$ & $<0.001$ \\
\hline
\end{tabular}

\begin{tabular}{|c|c|c|c|c|c|c|c|c|c|}
\hline $\operatorname{Pr}>=12 w$ & 3842 & 4183 & 4298 & 4475 & 4771 & 5001 & 4986 & & \\
\hline$\%$ & 7.6 & 8.1 & 8.6 & 9.1 & 9.8 & 10.4 & 10.9 & $+3.4 \%$ & $<0.001$ \\
\hline MSS & 1227 & 1414 & 1404 & 1396 & 1559 & 581 & 1651 & & \\
\hline$\%$ & 31.9 & 33.8 & 32.7 & 31.2 & 32.7 & 31.6 & 33.1 & $+0.2 \%$ & 0.84 \\
\hline FK total & 1703 & 1932 & 1852 & 1915 & 2142 & 2218 & 2230 & & \\
\hline$\%$ & 44.3 & 46.2 & 43.1 & 42.8 & 44.9 & 44.4 & 44.7 & $+0.1 \%$ & 0.93 \\
\hline FK only & 1233 & 1472 & 1469 & 1513 & 1722 & 1837 & 1867 & & \\
\hline$\%$ & 32.1 & 35.2 & 34.2 & 33.8 & 36.1 & 36.7 & 37.4 & $+4.5 \%$ & $<0.001$ \\
\hline $\mathbf{M S S}+\mathbf{F K}$ & 470 & 460 & 383 & 402 & 420 & 381 & 363 & & \\
\hline$\%$ & 12.2 & 11 & 8.9 & 8.9 & 8.8 & 7.6 & 7.3 & $-4.7 \%$ & $<0.001$ \\
\hline No MSS or FK & 1382 & 1297 & 1425 & 1566 & 1490 & 1583 & 1466 & & \\
\hline$\%$ & 36 & 31 & 33.2 & 35 & 31.2 & 31.7 & 29.4 & $-4.4 \%$ & $<0.001$ \\
\hline \multicolumn{10}{|l|}{$\leq 35$ years } \\
\hline $\operatorname{Pr}>=12 w$ & 46949 & 47598 & 45763 & 44966 & 43731 & 42881 & 40891 & & \\
\hline$\%$ & 92.4 & 91.9 & 91.4 & 90.9 & 90.2 & 89.6 & 89.1 & $-3.4 \%$ & $<0.001$ \\
\hline MSS & 20348 & 23230 & 23143 & 22713 & 23534 & 24218 & 23606 & & \\
\hline$\%$ & 43.3 & 48.8 & 50.6 & 50.5 & 53.8 & 56.5 & 57.7 & $+13.3 \%$ & $<0.001$ \\
\hline FK total & 2223 & 2397 & 2214 & 2319 & 2161 & 2383 & 2207 & & \\
\hline$\%$ & 4.7 & 5 & 4.8 & 5.2 & 4.9 & 5.6 & 5.4 & $+0.7 \%$ & $<0.001$ \\
\hline FK only & 954 & 988 & 979 & 1023 & 966 & 967 & 923 & & \\
\hline$\%$ & 2 & 21 & 2.1 & 2.3 & 2.2 & 2.3 & 2.3 & $+0.2 \%$ & 0.002 \\
\hline MSS + FK & 1269 & 1409 & 1235 & 1296 & 1195 & 1416 & 1284 & & \\
\hline$\%$ & 2.7 & 3 & 2.7 & 2.9 & 2.7 & 3.3 & 3.1 & $+0.4 \%$ & $<0.001$ \\
\hline No MSS or FK & 25647 & 23380 & 21641 & 20740 & 19231 & 16280 & 16362 & & \\
\hline$\%$ & 54.6 & 49.1 & 47.3 & 46.1 & 44 & 38 & 40 & $-14.9 \%$ & $<0.001$ \\
\hline
\end{tabular}

* Cochrane-Armitage Test for Trend (SAS system, SAS Inst Inc, Cary, NC USA) 
of both MSS $(+13.3 \%)$ and FK $(+0.7 \%)$, including a small increase of FK following MSS $(+0.4 \%)$. In both maternal age groups, the proportion of pregnancies without MSS or FK decreased markedly.

The overall estimated linear trend of $+1.9 \%$ of FK from 1995 to 2001 was thus mainly the result of the marked increase of FK only at $\geq 35$ years of age, associated with a concomitant decrease of FK following MSS in this group, plus a smaller increase of FK following MSS at $<35$ years of age. Figure 1 shows this evolution graphically: the only increasing fraction of FK between 1995 and 2002 was FK without MSS at maternal age $\geq 35$ years.

Figure 2.1. The evolution of fetal karyotyping (FK) between 1995 and 2002 , as registered by CM: FK with and without prior maternal serum screening (MSS) at $<35$ years or at $\geq 35$ years is expressed as a fraction of the total number of FK.

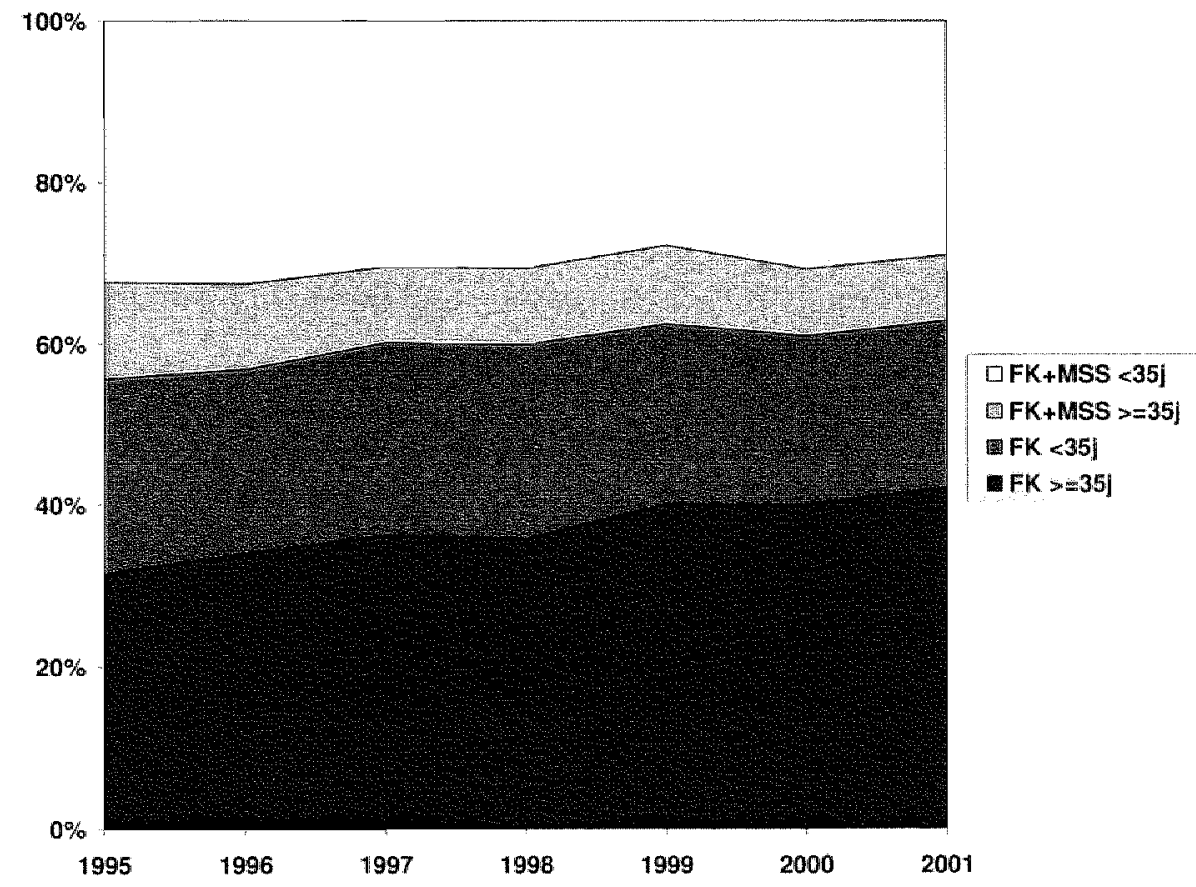

These data show an overall increase of fetal aneuploidy screening activities in Belgium between 1995 and 2002. Despite the excellent results of MSS at advanced maternal age, as discussed in the previous chapter, no increase of 
MSS or FK following MSS was observed in the CM population between 1995 and 2002. It seems unlikely that in the advanced maternal age group other screening methods, such as ultrasound screening, were performed instead of serum screening, as the total number of diagnostic invasive procedures for the indication of ultrasound parameters registered in Louvain was much lower than primary invasive testing for advanced maternal age. These data suggest that invasive tests (amniocentesis or chorionic villus sampling) seemed to be preferred over serum screening tests for women $\geq 35$ years of age whereas this was opposite at $<35$ years. In view of the data discussed in the previous chapter, this evolution looked rather controversial and this phenomenon initiated the onset for the study presented in this thesis.

\subsection{Aims of the study.}

In the study presented in this thesis, we set out to investigate four basic questions on letal aneuploidy screening practice in Flanders / Belgium:

1. Which are the different fetal aneuploidy screening methods available in Flanders / Belgium and do they have the same sensitivity (detection rate) and specificity (false positive rate) as reported in literature?

2. Can the optimal fetal aneuploidy screening method in Flanders / Belgium be identified, from the point of view of:

- the individual patient

- the population

3. Is it possible to identify any strategies, which improve the detection rate and reduce the false positive rate and subsequent invasive testing of unaffected pregnancies in Flanders / Belgium?

4. Is the current registration and follow up of screening practice in Flanders / Belgium sufficient to guarantee a satisfactory audit and quality control of performance, and to guide future optimisation of screening? 
Chapter 3

\section{Materials and methods}




\section{Summary}

Some of the basic principles of fetal aneuploidy screening are discussed: the use and relevance of different types of frequency distribution curves in affected and unaffected pregnancies, the definition of MoM- or Delta-values, detection rate, false positive rate, cut off value for positive screening and likelihood ratio.

The organisation of the fetal aneuploidy screening program by Algemeen Medisch Laboratorium in Antwerp is discussed for the development and validation of the screening algorithms, the collection and filing of data on pregnancy outcome, the interpretation of screening results and the methodology towards audit, quality control and optimisation of screening. 


\subsection{Basic principles of screening.}

The basic principles of screening for fetal aneuploidy have been described into detail in the book "Antenatal and neonatal screening" edited by Wald and Leck (Cuckle and Wald, 2000; Wald and Hackshaw, 2000). We briefly summarize the most important features we used to develop the algorithms from the Algemeen Medisch Laboratorium (AML) in Antwerp, Belgium, which are presented in the second part of this chapter.

\section{Expression of the measured values in relation to gestational age.}

Serum concentrations of biochemical parameters or measurements of ultrasound parameters change throughout the course of pregnancy. This means that one specific measurement may well be within the normal range at certain stages of gestation, but may be totally abnormal outside this gestational window. Therefore, all measured values are expressed as a function of the reference median value at this particular gestation, either as a multiple (Multiple of the Median $=$ MoM $)$ or as a difference $($ Delta $=\Delta)$. At a given gestational age, every MoM- or $\Delta$-value is a unique figure, which can be used for implementation into mathematic risk calculation algorithms.

Figure 3.1. Example of the frequency distribution of measured values of an arbitrary screening parameter in unaffected pregnancies and in pregnancies affected by fetal Down"s Syndrome.

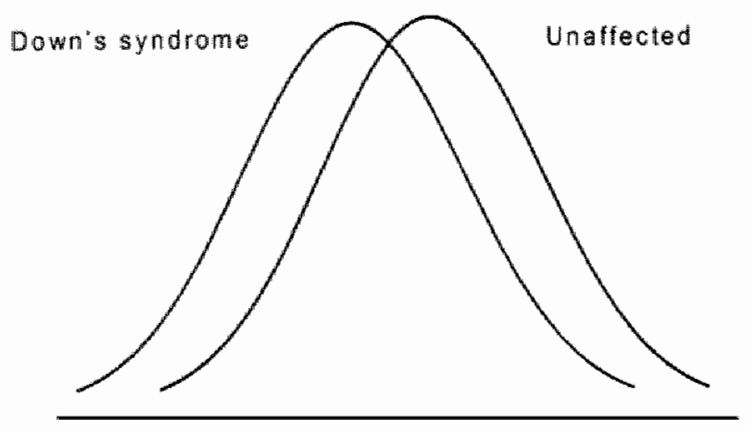

Test variable (arbitrary units) 


\section{Frequency distribution of MoM- or $\Delta$-values in affected and unaffected}

pregnancies.

For the purpose of screening, only those parameters are useful of which the frequency distribution of measured values is different in affected and nonaffected pregnancies. These distribution curves are necessary to define the chance or odds that a particular measured value belongs to the affected or the unaffected population. This principle is shown in Figure 1.

The distribution curves in the affected and the unaffected pregnancies are derived from empirical observations: Figure 2 shows the frequency distribution of NT measurements in 26 trisomy 21 ("T21)-affected pregnancies and 13181 unaffected pregnancies of our population. Values are expressed as $\Delta N T$ or logarithmic $_{10}$ transformed NT-MoM- values (LogNT-MoM). Note that the $\Delta$ NT distribution is non-gaussian and the LogNT-MoM curve is very close to a normal gaussian distribution. We used the LogMoM values for both the serum and ultrasound parameters in our algorithm.

From the distribution curves in the affected and unaffected pregnancies, it is clear that the value of any parameter as a screening tool depends on two important characteristics:

- the difference between the median values in the affected and unaffected populations: the bigger this difference, the better this parameter discriminates between affected and unaffected populations. As is shown in Figure 3, parameter (iii) is more useful for screening than parameter (i).

- The standard deviation of the distribution in the affected and unaffected populations: a small standard deviation is a reflection of the consistency of a particular value for being close to the median of the affected or the unaffected population. In Figure 3 , the parameter of which the distribution curves are presented in interrupted lines, is more useful for screening than the parameter, which distribution curves are shown in continuous lines. 
Figure 3.2. Example of the distribution of Nuchal Translucency thickness measurements in the unaffected population (solid line) and Down's Syndrome affected pregnancies (interrupted line). NT measurements are copressed as Delta-NT (upper) or as LogNT-MoM (lower). The LogNT-MoM curve is closer to a gaussian distribution than the Delta-NT curve.
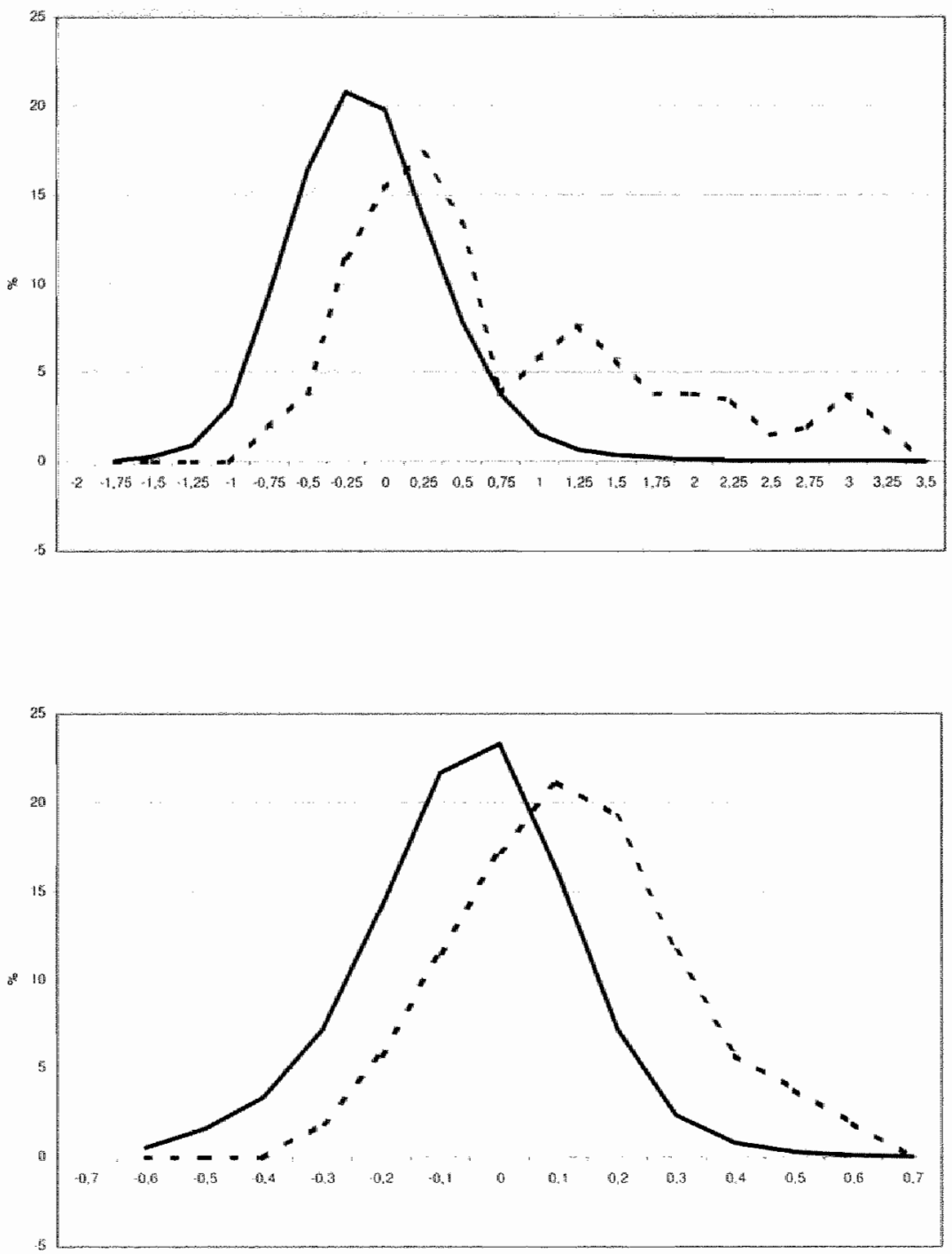
Figure 3.3. Example of frequency distribution curves with and $(0)$ and farge (1in) difference in the values of the affected and unaffected population, and with andl (interrupted line) and a large (solid line) standard deviation (reproduced with permission from Cucle and Wald, 2000).
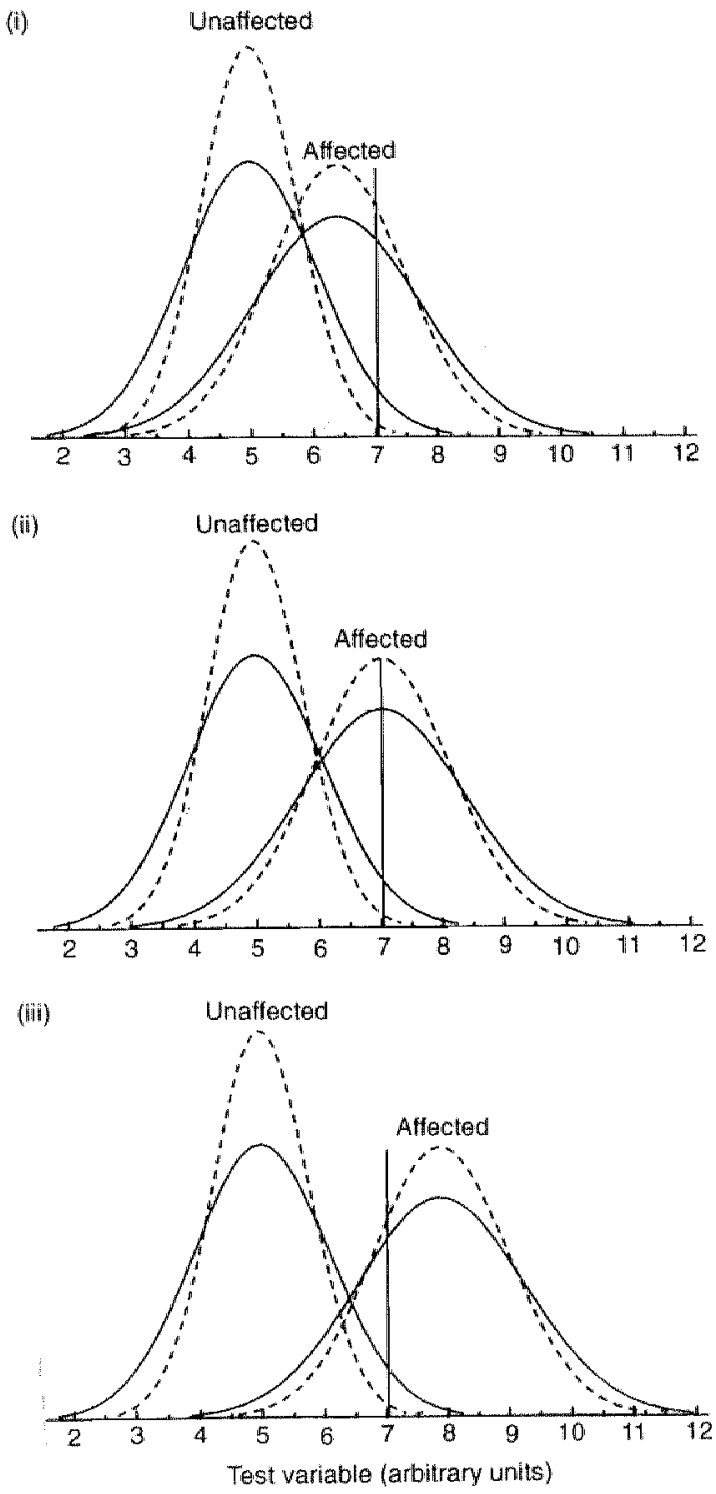

Figure 4 shows the distribution of the LogNT-MoM values in our population: as is shown, the standard deviation of the curve of NT- 
measurements, performed by the ultrasonographers who were trained by The Fetal Medicine Foundation (FMF), is smaller than the standard deviation of the curve of NT-measurements by nonFMF-trainees. Therefore, the NT measurements of the FMFtrainees will perform better as a screening parameter than those of the other ultrasonographers.

Figure 3.4. Frequency distribution of the LogNT-MoM values in the Down's Syndrome pregnancies ( (...) and in the unaffected pregnancies, examined by ultrasonographers tranned by the

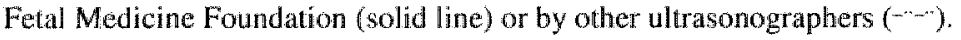

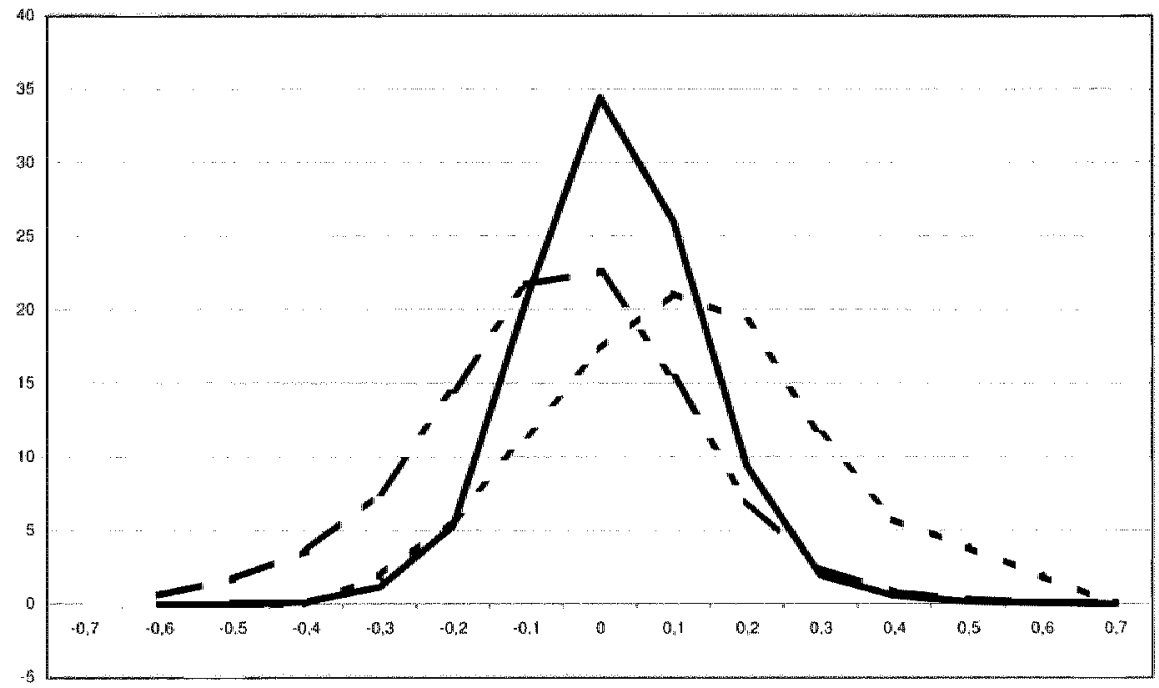

\section{Detection rate, false positive rate and cut off value.}

The result of a fetal aneuploidy screening test is considered "positive", when it indicates an increased risk for a chromosomally abnormal fetus. The screening result is considered "negative" when the probability for an unaffected pregnancy is high. The value, which discriminates positive from negative screening results, is called the "cut off point". This cut off point is at the level, where the chance or odds to detect an affected pregnancy is high (detection rate 
$=\mathrm{DR}$ ) and the probability to misjudge a normal pregnancy is low (false positive rate $=$ FPR). This is shown graphically in Figure 5. This figure clearly shows that there is a direct relationship between DR and FPR for every screening method: moving the cut off value towards a higher DR will automatically increase FPR and vice versa.

Figure 3.5. Illustration of the relationship between Detection Rate (DR), Fallse Positive Rate (FPR) and cut off level for positive screening (reproduced with permission from Cuckle and Wald, 2000).

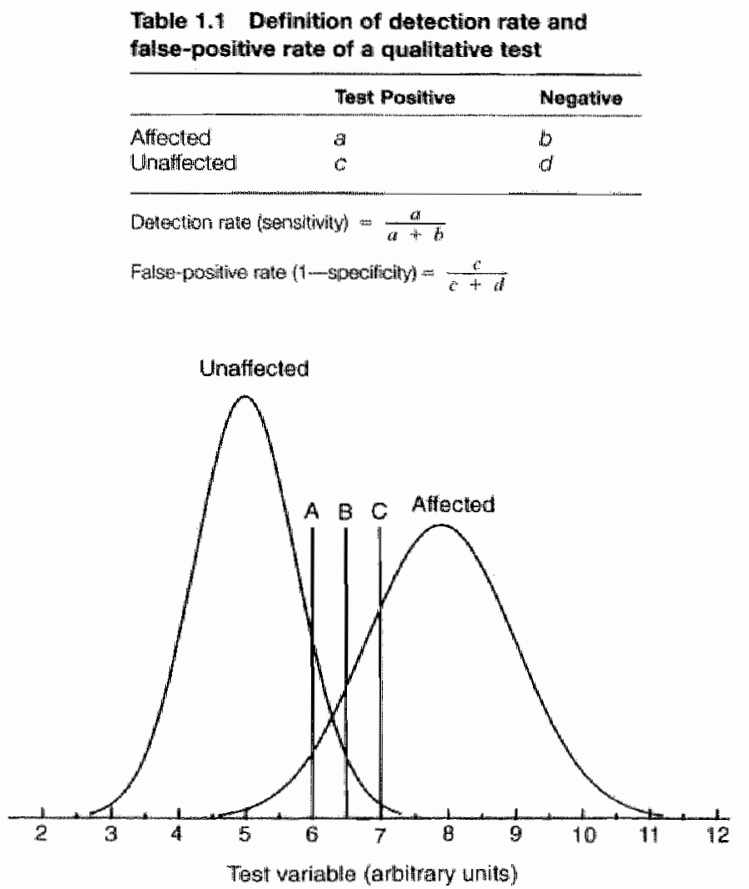

Fig. 1.2. Hypothetical exiample of the detection rate and false-positive rate of a screening test at three different Cull-off llewels, $A_{v} B$, and $C$

\begin{tabular}{lll}
$\begin{array}{l}\text { Cui-off } \\
\text { level }\end{array}$ & $\begin{array}{l}\text { Detection rate } \\
(\%)\end{array}$ & $\begin{array}{l}\text { Falso-positive rate } \\
(\%)\end{array}$ \\
\hline A & 96 & 10.0 \\
B & 90 & 2.5 \\
C & 79 & 0.5
\end{tabular}


The relation between DR and FPR can also be illustrated graphically in a ROCcurve: Figure 6 shows for the AML-algorithms the ROC-curves for second trimester and for first trimester screening.

Figure 3.6. ROC-curves of the AML second and first trimester screening algorithms, illustrating the relation between Detection Rate (sensitivity) and False Positive Rate (1 specificity).

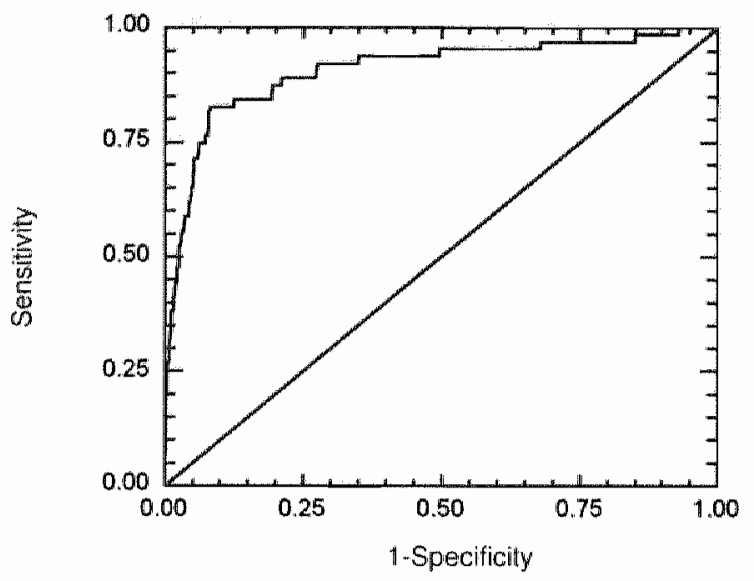

\section{Second trimester triple}

serum screening

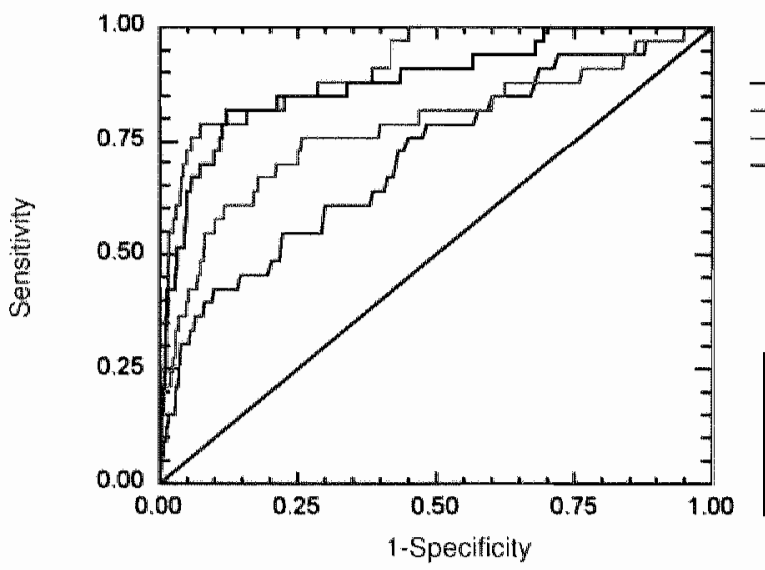

First trimester combined screening

Age

Age+NT

Combined

Agotserum 
The cut off value may be defined at a different level, according to the type of study performed:

- Clinical studies: for every screening method, the cut off point for positive screening is defined at the level where the balance between DR and FPR is considered "optimal". In the AML- second trimester maternal serum screening algorithm in 1992, this cut off level was defined at 1:300. For practical reasons this value was maintained also for the first trimester screening algorithms, which were developed 7 to 8 years later. Table 1 shows the DR and FPR at cut off 1:300 for each of second and first trimester algorithms.

- Performance studies: For comparison of the performance of different screening methods, standardisation to express the screening results is needed. Usually, the FPR is fixed (e.g. 5\%) and the difference in DR at this particular level compared between the different screening methods. Similarly, the DR can be fixed (e.g. 85\%) and the difference in FPR at this particular level is compared between the different screening methods. Table 2 shows the DR and the cut off values for positive screening at a fixed $5 \%$ FPR for the AML-second and first trimester screening algorithms.

Table 3.1. Trisomy 21 (T21) detection rate and false positive rate at a cut of level of 1:300 of 4 different AML-screening algorithms: second trimester maternal serum screening with AFP. HCG and $\mathrm{EE}_{3}$ (2MSS), first trimester maternal serum screening with $\mathrm{PAPP}-\mathrm{A}$ and $\mathrm{FB}-\mathrm{HCG}$ (IMSS), first trimester NT screening (NT) and first trimester combined screening (IMSS + NT).

\section{T21-affected pregnancies}

\section{MSS $<=1998$ 1MSS NT 1MSS+NT}

$\begin{array}{ccccc}\mathbf{n} \text { total } & 60 & 26 & 26 & 26 \\ \mathbf{n}>=1: 300 & 44 & 21 & 11 & 21 \\ \text { Detection rate }(\%) & 73 & 81 & 42 & 81\end{array}$

\section{Unaffected pregnancies}

\begin{tabular}{lcccc}
$n$ total & 40349 & 13181 & 13181 & 13181 \\
$n>=1: 300$ & 2291 & 2212 & 635 & 1130 \\
se positive rate (\%) & 5.7 & 16.8 & 4.8 & 8.6 \\
\hline
\end{tabular}


Table 3.2

Detection Rate and cut off levels at fixed $5 \%$ false positive rates of 4 different AML-screening algorithms: second trimester maternal serum screening with $A F P$. HCG and $u E_{3}$ (2MSS), first trimester material serum screening with PAPP-A and FB-HCG (IMSS), first trimester NT screening (NT) and first trimester combined screening (IMSS + NT).

\section{$2 M S S<=1998 \quad 1$ MSS NT 1 MSS + NT}

\section{T21-affected pregnancies}

$\begin{array}{ccccc}\mathbf{n} \text { total } & 60 & 26 & 26 & 26 \\ \mathbf{n}>=1: 300 & 40 & 18 & 11 & 19 \\ \text { Detection rate (\%) } & 67 & 62 & 42 & 74\end{array}$

\section{The likelihood for being affected and the likelihood ratio.}

For every screening parameter, the distribution curves in the affected and unaffected population can be used to define the likelihood that a particular measured value belongs to an affected or an unaffected pregnancy. This likelihood is derived from the balance between DR and FPR, and the likelihood ratio (LR) is defined as DR/FPR. To calculate an individual patient's risk for being affected, this LR is multiplied by the individual's background risk. This background risk depends on the disease screened for, and the individual's personal characteristics. For fetal Down's Syndrome screening, the pregnant woman's background risk is basically related to her age and the gestation at which the parameter is sampled (Morris et al., 2002;Snijders et al., 1999). In multiple parameter sampling, the LR's derived for every parameter are multiplied with each other and with the background risk to calculate the final screening result. In these multiple parameter algorithms, corrections are needed to compensate for correlations between the different parameters used (Wald and Hackshaw, 2000), 


\subsection{The fetal aneuploidy screening program organised by the Algemeen Medisch Laboratorium (AML).}

The majority of data presented in this thesis is from the database on fetal aneuploidy screening, as registered by the Algemeen Medisch Laboratorium (AML) in Antwerp, Belgium.

AML was founded in 1975 as a private medical laboratory, and soon became specialised in laboratory technologies for obstetricians, gynaecologists and infertility clinics. During its 30 -years history, it has grown continuously to become the largest private lab in Flanders. AML was the first laboratory in Flanders to introduce second trimester maternal serum screening in 1991 and first trimester combined screening in 2000. During this 14-years period, over 100.000 pregnancies have been screened, and this experience is reflected in one of two large databases on fetal aneuploidy screening currently available in Belgium (Vamos et al., 1997). A continuing update and expansion of this database is achieved through collaboration of over 250 obstetricians, active in 35 out of 75 maternity clinics in Flanders from all regions of the country: of a total number of 96815 screening tests performed at the end of 2003,31464 (32.5\%) were from Antwerp, 27884 (28.8\%) were from Limburg, 14038 (14.5\%) were from Brussels, 12683 (13.1\%) were from Brabant and 10746 $(11.1 \%)$ were from East- and West-Flanders.

In the following part of this chapter, we discuss the AML methodology for:

1. the development of the screening algorithms

2. the collection and filing of data on pregnancy outcome

3. the validation of the screening algorithms

4. the interpretation of the screening results

5. audit, quality control and optimisation of the screening program 


\subsubsection{The development of the screening algorithms.}

The development of the fetal aneuploidy screening algorithm using second trimester maternal serum parameters was initiated in 1991 and further refined in the following years. The basis for the AML- algorithm was the publication of Wald and his colleagues in 1988 (Wald et al., 1988). The mathematic algorithm reported in that paper was set out in a simple form by Palomaki, and distributed for free on the condition of acknowledgement (G. Palomaki, 1992, Foundation for Blood Research, Box 190, Scarborough, ME 04074, United States).

Second trimester maternal serum samples were analysed for concentrations of $\alpha$-Feto Protein (AFP), total $\beta$-Human Chorionic Gonadotropin (B-HCG) and unconjugated estriol $\left(\mathrm{uE}_{3}\right)$ using isotopic assays $\left(\mathrm{I}^{125}\right)$, developed and distributed by respectively Diagnostic Products Corporation (Los Angeles, CA, USA), BioSource Europe SA (Nivelles, Belgium) and Diagnostic Systems Laboratories Inc (Webster, TX, USA). Results were expressed in multiples of the median (MoM) for the appropriate gestation and logarithmic 10 values were used for risk calculations (Wald et al., 1988). Concentrations of $\mathrm{uE}_{3}$ were expressed in MoM without logarithmic transformation. Screening for fetal aneuploidy was performed twice a week ( $\geq 80$ samples/run) and all assays were performed in duplicate. After each run, quality control was performed by calculation of the means, medians and standard deviations for each parameter. MoM-values were controlled each month with an exponential regression analysis (NCSS Statistical Software, 329 North 1000 East-Kaysville, Utah 84037, United States) and adjusted when necessary. Correction was performed for weight (Bartels et al, 1993), insulin dependent diabetes (Palomaki et al., 1994), twins (Wald and Densem, 1994a; Wald and Densem, 1994b), smoking (Bartels et al., 1993) and ethnicity (Watt et al., 1996). Ultrasound estimated dates of delivery were used to define gestational age at blood sampling correctly (Wald et al. 1992). 
When enough data on pregnancy outcome were available $\geq 30$ cases of trisomy 21), population specific medians and inter-paraneter correlations were calculated from a group of affected and unaffected pregnancies.

In 1996, AML also started to collect data on the ultrasonic measurement of fetal crown-rump length (CRL) and nuchal translucency thickness (NT) in the first trimester of pregnancy. The obstetricians, who referred the maternal serum samples for fetal aneuploidy screening, performed these measurements. No details were provided on the methodology of NT measurement. A total of 2700 NT/CRL measurements were mailed to The Fetal Medicine Foundation (FMF). From these data, CRL-related NT-MoM values were mailed back, which were used to establish the FMF-specific reference curve of gestation- related NT values. From this, NT-MoM values relative to the FMF- reference range could be defined for every NT measurement in the database. These values were used to calculate the risk for fetal aneuploidy, using the likelihood ratio's (LR) reported by Pandya et al. (Pandya et al., 1995; Snijders and Nicolaides, 1996). Considering no correlation between first trimester NT and second trimester maternal serum parameters, these likelihood ratio's were also used to combine or integrate NT with second trimester triple serum screening. Risk calculations from this expanded algorithm were introduced into clinical practice in 1998.

In 2000, AML also started to measure first trimester concentrations of Pregnancy Associated Plasma Protein A (PAPP-A) by enzyme-linked immunosorbent assay (Eliza 2397, DRG International Inc, USA), and free $\beta$ HCG (FB HCG) by radio-immuno assay (HCG IRMA KIPI001, BioSource Europe SA, Nivelles, Belgium). Each result was expressed as MoM, adjusted for maternal weight (de Graaf et al., 1999), multiple gestation (Spencer, 2000), smoking (de Graaf et al., 2000) and ethnicity (Hallahan et al., 2004). Similar to the AML second trimester algorithm, the means, medians, standard deviations and MoM-values of PAPP-A and FB HCG were controlled each month with an exponential regression analysis using NCSS statisticall software and adjusted when necessary. Likelihood ratio's and parameter correlations as reported by de 
Graaf et al (de Graaf et al., 1999) were used in the same algorithm as described above (Wald et al., 1988). First trimester screening, combining maternal serum parameters with NT, was introduced into clinical practice in 2001.

Figure 7 shows the evolution of different screening tests performed by AML. between 1992 and 2004.

Figure 3.7. The evolution of 3 different screening methods, as registered by AML between 1992 and 2003 : second trimester maternal serum screening with $\mathrm{AFP}$, HCG and $\mathrm{uE}_{3}$ (2MSS), 2MSS with first trimesiter NT (2MSS+NT) and first trimester combined screening (IMSS + NT)

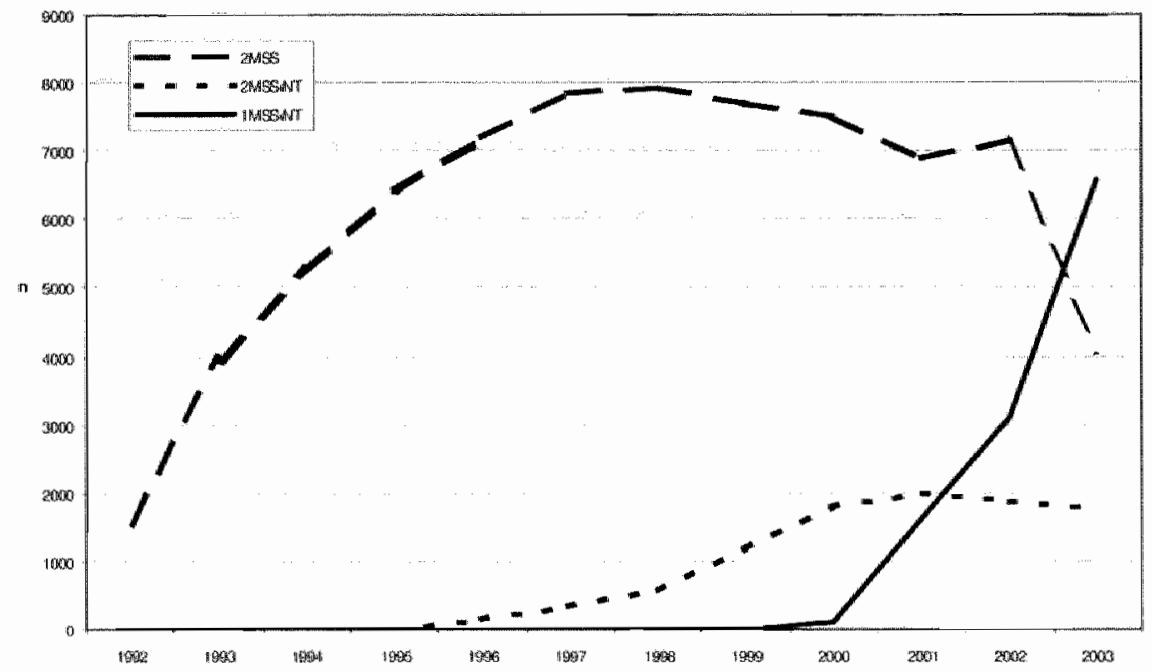

\subsubsection{The collection and filing of data on pregnancy outcome.}

In 2003, a total of 264 obstetricians participated in the fetal aneuploidy screening program organised by AML. At least once a year, all obstetricians reported the results of invasive procedures and/or the outcome of pregnancies by mail. Non-responding obstetricians were contacted personally to collect missing data on chromosomal anomalies. Despite these efforts, there was only a 
response rate of $51.7 \%$ of all pregnancies screened (Gyselaers et al., 2004). To reduce the workload of the reporting process for the obstetricians, it was agreed to only report the abnormal outcome of the screened pregnancies, both in terms of fetal (chromosomal or structural) abnormalities and of abnormal clinicall outcome. All data were filed into an electronic database.

For the study on screening for fetal trisomy 21 (T21) reported in this thesis, data were grouped in T21-affected pregnancies, pregnancies affected by fetal chromosomal anomalies different from $\mathrm{T} 21$, pregnancies with spontaneous fetal loss of unknown genetic constitution, and unaffected pregnancies.

The registration method was validated by comparing the prevalence of $T 21$ at term, as expected from the maternal age distribution in our population and the data on maternal age- and gestation- related prevalence, as registered in the British National Down's Syndrome Cytogenetic Register (BNDSCR) (Morris et al., 1999; Morris et al., 2002). For this, the maternal age- and gestation-related prevalence of $\mathrm{T} 21$ was assumed to be similar in Belgium and the United Kingdom. All data were grouped according to the time period of screening and the type of screening method: (1) pregnancies screened by second trimester maternal serum parameters between 1992 and 1999, (2) pregnancies screened by second trimester maternal serum parameters between 1999 and 2004 without any information on NT measurement, (3) pregnancies screened second trimester maternal serum parameters between 1999 and 2004 with information on NT measurement and (4) pregnancies screened by NT and first trimester maternal serum parameters. The calculation of the expected number of T21-affected births in these 4 groups is shown in Table 3. For each group, the number of screened pregnancies per year of maternal age was multiplied with the maternal age related prevalence reported in the BNDSCR. By summation, the total number of T21-affected pregnancies expected at birth could be estimated for each group. 
MA EP T21 Pregn ExpT21 Pregn ExpT21 Pregn ExpT21 Pregn ExpT21

\begin{tabular}{|c|c|c|c|c|c|c|c|c|c|}
\hline (y) & $1: x$ & $\mathrm{n}$ & $n$ & $n$ & $n$ & $\mathrm{n}$ & $n$ & $\mathrm{n}$ & $\mathrm{n}$ \\
\hline$<15$ & 1516 & 3 & 0,002 & 1 & 0 & 1 & 0 & 2 & 0,001 \\
\hline 15 & 1513 & 8 & 0,005 & 3 & 0,002 & 3 & 0,002 & 2 & 0,001 \\
\hline 16 & 1509 & 37 & 0,024 & 15 & 0,01 & 8 & 0,005 & 3 & 0,002 \\
\hline 17 & 1504 & 79 & 0,053 & 51 & 0,034 & 22 & 0,015 & 21 & 0,014 \\
\hline 18 & 1.497 & 209 & 0,14 & 106 & 0,071 & 35 & 0,023 & 65 & 0,043 \\
\hline 19 & 1488 & 341 & 0,229 & 262 & 0,176 & 87 & 0,058 & 117 & 0,079 \\
\hline 20 & 1.476 & 528 & 0,358 & 439 & 0,297 & 139 & 0,094 & 191 & 0,129 \\
\hline 21 & 1461 & 759 & 0,52 & 622 & 0,426 & 188 & 0,129 & 243 & 0,166 \\
\hline 22 & 1441 & 1151 & 0,799 & 859 & 0,596 & 251 & 0,174 & 336 & 0,233 \\
\hline 23 & 1415 & 1717 & 1,213 & 1062 & 0,751 & 347 & 0,245 & 517 & 0,365 \\
\hline 24 & 1381 & 2443 & 1,769 & 1444 & 1,046 & 453 & 0,328 & 651 & 0,474 \\
\hline 25 & 1339 & 3249 & 2426 & $18: 10$ & 1,352 & 559 & 0,417 & 811 & 0,606 \\
\hline 26 & 1285 & 3821 & 2,974 & 2254 & 1,754 & 633 & 0.493 & 1007 & 0,784 \\
\hline 27 & 1219 & 4237 & 3,476 & 26,10 & 2,1141 & 787 & 0,646 & 1138 & 0,934 \\
\hline 28 & 1139 & 4194 & $3,68: 2$ & 3027 & 2,658 & 752 & 0,66 & 1181 & 1,037 \\
\hline 29 & 1045 & 3929 & 3,76 & 333.1 & 3,188 & 792 & 0,758 & 1237 & 1,184 \\
\hline 30 & 937 & 3359 & 3,585 & 3314 & 3,537 & 800 & 0,854 & 1199 & 1,28 \\
\hline 31 & 819 & 2803 & 3,422 & 3166 & 3,866 & 658 & 0,803 & 1105 & 1,349 \\
\hline 32 & 695 & 2257 & 3,247 & 2660 & 3,827 & 540 & 0.777 & 880 & 1,266 \\
\hline 33 & 571 & 1807 & 3,165 & 2157 & 3,777 & 428 & 0,75 & 709 & 1,242 \\
\hline 34 & 455 & 1346 & 2,958 & 1824 & 4,009 & 353 & 0,776 & 581 & 1,277 \\
\hline 35 & 352 & 803 & 2,28 & 1416 & 4,023 & 245 & 0,696 & 421 & 1,196 \\
\hline 36 & 266 & 513 & 1.929 & 919 & 3,455 & 151 & 0,568 & 291 & 1,094 \\
\hline 37 & 199 & 347 & 1.744 & 550 & 2,764 & 97 & 0,487 & 204 & 1,025 \\
\hline 38 & 148 & 201 & 1,358 & 326 & 2,203 & 53 & 0,358 & 139 & 0,939 \\
\hline 39 & 111 & 125 & 1,126 & 177 & 1,595 & 36 & 0,324 & 92 & 0,829 \\
\hline 40 & 85 & 58 & 0,682 & 127 & 1,494 & 27 & 0,318 & 57 & 0,671 \\
\hline 41 & 67 & 48 & 0,716 & 67 & 1 & 14 & 0,209 & 35 & 0,522 \\
\hline 42 & 54 & 21 & 0,389 & 36 & 0,667 & 9 & 0,167 & 19 & 0,352 \\
\hline 43 & 45 & 11 & $0,24.4$ & 25 & 0,556 & 1 & 0,022 & 8 & 0,178 \\
\hline 4.4 & 39 & 1 & 0,026 & 7 & 0,179 & 0 & 0 & 4 & 0,103 \\
\hline 45 & 35 & 1 & 0,029 & 1 & 0,286 & 0 & 0 & 1 & 0,029 \\
\hline 46 & 31 & 1 & 0,032 & 1 & 0,032 & 0 & 0 & 0 & 0 \\
\hline 47 & 29 & 1 & 0,034 & 1 & 0,034 & 0 & 0 & 0 & 0 \\
\hline 48 & 27 & 1 & 0,037 & 0 & 0 & 0 & 0 & 0 & 0 \\
\hline 49 & 26 & 0 & 0 & 0 & 0 & 0 & 0 & 0 & 0 \\
\hline 50 & 25 & 0 & 0 & 0 & 0 & 0 & 0 & 0 & 0 \\
\hline Total & & 40409 & 48,433 & 34670 & 51,806 & 8469 & 11,156 & 13267 & 19,401 \\
\hline
\end{tabular}


Table 3.3. Calculation of the expected number of Down's Syndrome affected births (ExpT21) using the data of the British National Down's Syndrome Cytogenetic Register (Morris EP T21,(Morris ef al., 2002)) and the number of pregnancies (pregn) per year of naternal age (MA) in the AML populations, screened by second trimester maternal serum (2MSS) with or whthout information on NT, and by first trimester combined screening (IMSS + NT). This method was reported by Wald et al (Wald and Hackshaw, 2000).

Table 4 shows the number of T21-affected pregnancies, as observed in each of the 4 groups of the AML population. Because many of the observed cases of Down's Syndrome were diagnosed during the course of the pregnancies as a result of positive screening and subsequent invasive diagnostic procedures, we had to correct our observed data for the spontaneous fetal loss rate expected between the first or second trimester and birth assuming no interventions would be performed. For this, we also used the BNDSCR-data published by Morris et al. (Morris et al., 1999), who reported for T21-affected pregnancies a $43 \%$ fetal loss rate between 12 weeks and term and a $23 \%$ fetal loss rate between 16 weeks and term. We defined the number of expected births from this group as $77 \%$ of the number of interrupted second trimester pregnancies and $57 \%$ of the number of interrupted first trimester pregnancies. This correction of our observed data is shown in Table 4 , and the results reflect the number of T21affected newborns in our population if no interventions or terminations of pregnancies would have occurred. This correction allowed for correlation of the observed numbers of T21-affected births with the number expected from the characteristics of the screened population, as explained above. The correlation coefficient for second trimester matemal serum screening $\leq 1998$ was 1.04 , for second trimester maternal serum screening $\geq 1.999$ was 0.69 , for second trimester maternal serum screening and NT was 0.86 and for first trimester maternal serum screening and NT was 0.96 (Table 4). These data indicate a good correlation for second trimester maternal serum screening $\leq 1998$, for second trimester maternal serum screening + NT between 1999 and 2004, and for first trimester combined screening. This illustrates that our method of data collection and registration is valid. However, the correlation for second 
trimester maternal serum screening between 1999 and 2004, without information on NT measurement, is poor for second trimester maternal serum screening $\geq 1999$ : it is clear that in this group many expected cases of Down's Syndrome were missing in our database. The most likely explanation for this is a partial elimination from the population through first trimester ultrasound screening, subsequent invasive testing and terminations of affected pregnancies. On questioning the obstetricians in Flanders about their screening policies, many of them reported sequential screening practice by first trimester ultrasound screening with referral of the pregnancies with abnormal screening results for invasive diagnostic testing. Subsequent second trimester maternal serum screening was only performed in those pregnancies with normal first trimester screening results. In most of the cases, first trimester NT measurements were not reported to the laboratory when maternal serum was sent for second trimester screening.

Table 3.4. Comparison between the expected ( $\mathrm{E}$ T21) and observed (O T21) Down's Syndrome affected births in 4 AML-populations, screened by different screening methods or in at different time period (see Table 3). The observed number of Down's Syndrome affected births is the sum of live births and the expecied number of live births from interrupted pregnancies, assuming no interventions were done. For this, a $23 \%$ spontaneous fetal loss rate from 16 weeks to term and $43 \%$ trom 12 weeks to term was considered (Morris ei al., 1999).

\section{Total $>1: 300<1: 300$ Interrup Live birth E T21* 0 T21 ${ }^{\circ} \mathrm{E} / \mathrm{O}^{* *}$}

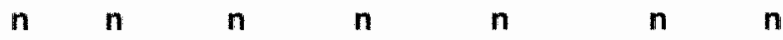

\begin{tabular}{lllllllll}
\hline 2MSS 1992-1998 & 60 & 44 & 16 & 42 & 18 & 48,43 & 50,34 & 1,04 \\
2MSS 1999-2003 & 42 & 26 & 16 & 25 & 17 & 51,81 & 36,25 & 0,69 \\
2MSS+NT 1999-2003 & 11 & 6 & 5 & 6 & 5 & 11,16 & 9,62 & 0,86 \\
1MSS+NT 2001-2003 & 26 & 21 & 5 & 17 & 9 & 19,4 & 18,69 & 0,96 \\
\hline
\end{tabular}

* Table I

Live births $+n$ interruptions $\times 77 \%(2 \mathrm{MSS}+/-\mathrm{NT})$

Live births $+\mathrm{n}$ interruptions $\times 57 \%$ (IMSS $+/-\mathrm{NT})$

*** $\mathrm{E} / \mathrm{O}=$ ratio of expected $(\mathrm{E} \mathrm{T} 21)$ over observed $(\mathrm{O} \mathrm{T} 21)$ number of $\mathrm{T} 21$ affected births

The practice of sequential screening is also clearly illustrated by the changing prevalence of $\mathrm{T} 21$-affected pregnancies in the populations screened by second 
trimester maternal serum screening: before 1999 this prevalence was $1 / 673$ (60/40409) and after 1999 this was only 1/827 (42/34670) (Table 3 and 4).

\subsubsection{The validation of the screening algorithms.}

An empirical method to evaluate the validity of the risk calculation algorithms was reported by Wald et al in 1996 (Wald et al., 1996) and Onda et al in 1998 (Onda et al., 1998). The algorithm"s calculated risk for T21 is compared to the observed prevalence of $\mathrm{T} 21$ in the screened population: a correlation coefficient near 1 indicates that the risks calculated by the algorithm are in agreement with reality and useful for clinical practice.

This method was used to validate the AML second trimester maternal serum algorithm and the first trimester combined algorithm. As shown in Figures $8 \mathrm{~A}$ and $8 \mathrm{~B}$, the correlation coefficient between the logarithmic values of the calculated and observed risks was 0.99 for second trimester screening and 0.93 for screening in the first trimester.

\subsubsection{Interpretation of screening results.}

At least once every year, the screening performance of the algorithms was evaluated by ROC-analysis, which depicts the balance between DR and FPR. As such, the evolution of the screening performance in time was evaluated and also the contribution of each parameter to the final screening result. Figure $6 \mathrm{~A}$ and $6 \mathrm{~B}$ show the ROC-curves for second trimester maternal serum screening $\leq$ 1998 and for first trimester combined screening. This information was communicated to the collaborating obstetricians by mailing of the AML Year Report on Fetal Aneuploidy Screening or by personal visits to the maternity clinics.

For both the second trimester maternal serum algorithm and the first trimester combined algorithm, AML initially defined the cut off level for positive screening empirically at the 5\% SPR of the first 5000 screening tests. When necessary, corrections were made following ROC-analysis on a larger set of data. Most obstetricians in Flanders currently define a calculated risk of $\geq$ 
$1: 300$ as the cut off level for positive screening, both in second trimester and first trimester screening. At a cut off level of 1:300, the FPR for second trimester maternal serum screening $\leq 1.998$ was $5.7 \%(2291 / 40349)$ and for first trimester combined screening this was $8.6 \%$ (1130/13181) (Table 1). From the information in the AML Year Reports on Fetal Aneuploidy Screening, some obstetricians spontaneously started to offer invasive testing only for a calculated risk $\geq 1: 200$ in the first trimester algorithm, at which FPR is 5.5\% (724/13181).

The performance of the different screening algorithms was compared by evaluation of the DR at a fixed 5\% FPR. Many publications on the performance of fetal aneuploidy screening algorithms also report this DR at fixed 5\% FPR, so this method also allowed us to compare our data with literature.

\subsubsection{Audit, quality control and optimisation of the screening program.}

As discussed above, quality control for maternal serum screening was performed after each analytical run in the lab. MoM-values were controlled each month with an exponential regression analysis (NCSS Statistical Software) and adjusted in the algorithm when necessary.

Audit on NT measurements was performed similar to the approach of FMF. Every NT measurement in the database was expressed as a $\triangle N T$-value, relative to the FMF-reference range. A frequency distribution curve of these $\triangle \mathrm{NT}$ values was established and compared to the FMF reference $5^{\text {th }}, 50^{\text {th }}$ and $95^{\text {th }}$ percentile, which were defined at $-0.45 \mathrm{~mm}, 0 \mathrm{~mm}$ and $+0.75 \mathrm{~mm}$ from the FMF-software for first trimester screening audit. These distribution curves were established for two groups of ultrasonographers: those who were enlisted as FMF-trainees at the official FMF-website (www fetalmedicine.com/fcompetence.htm) and those who were not on this list. The results of this audit are discussed into detail in Chapter 6.

The main objectives of this thesis are related to the audit results of the AML fetal aneuploidy screening program. The details of this audit, the results and the 
subsequent conclusions are handled into detail in the forthoming chapters. For reasons of comprehension, we summarise and discuss audit, quality control and optimisation of the screening program at the end of this thesis in Chapter 11 (General discussion and future perspectives).

References

Bartels, 1., Hoppe-Sievert, B., Bockel, B., Herold, S., and Caesar, \#. (1993) Adjustment formulae for matemal serum alpha-fetoprotein, human chorionic gonadotropin, and unconjugated oestriol to maternal weight and smoking. Prenat Diagn 13:123m 130

Cuckle,H. and Wald,N. (2000) Priciples of screening: tests using single markers. In Antenatal a Neonatal screening. Wald, N. and Leck,I. (eds.) Oxford: Oxford University Press, pp. 3-22.

de Graaf, I. M., Cuckle, H. S., Pajkrt, E., Leschot, N. J., Bleker, O. P., and van Lith, J. M. (2000) Co-variables in first trimester matemal serum screening. Prenat Diagn 20:186-189.

de Graaf, I. M., Pajkrt, E., Bilardo, C. M., Leschot, N. J, Cuckle, H. S., and van Lith, J. M. (1999) Early pregnancy screening for fetal aneuploidy with serum markers and nuchal translucency. Prenat Diagn 19: 458-462.

Gyselaers, W., Vereecken, A., Van Herck, E., Straetmans, D., Martens, G., De Jonge, E. Ombelet, W., and Nijhuis, J. (2004) Screening for trisomy 21 in Flanders: a 10 -years review of 40490 pregnancies screened by matenal serum. Eur J Obstet Gynecol Reprod Biol 115: 185-189.

Hatlahan, T., Krantz, D., Buchanen, P.. Larsen, J. W., and Macri, J. Effect of ethnicity of fursttrimester Down Syndrome screening. hhtp:/genetics.faseb.org/genetics/ashgo0/ $2365 . \mathrm{htm}$, (accessed 20-5/2004).

Morris, J. K, Mutton, D. E., and Alberman, E. (2002) Revised estimates of the maternal age specific live birth prevalence of Down's syndrome. A Med Screen 9: 2-6.

Morris, J. K., Wald, N. J., and Watt, $H$. C. (1999) Fetal loss in Down syndrome pregnancies. Prenat Diagn 19:142-145.

Onda, T., Tanaka. T., Takeda, O., Kitagawa, M., Kuwabara, Y., Yamamoto, H. linuma, K., and Shimomura, K. (1998) Agreement between predicted risk and prevalence of Down syndrome in second-trimester triple-marker screening in Japan. Prenat Diagn $18: 956-958$.

Palomaki, G. E. Knight, G. J., and Haddow, J. E. (1994) Human chorionic gonadotropin and unconjugated oestriol measurements in insulin-dependent diabetic pregnant women being screened for fetal Down syndrome. Prenar Diagn 14:65-68.

Pandya, P. P.. Snijders, R. J., Johnson, S. P., De Louldes, Brizot M., and Nicolaides, K. H. (1995) Screening for fetal trisomies by matemal age and fetal nuchal translucency thickness at 10 to 14 weeks of gestation. BrJ Obstet Gyarecol J 02: $957-962$. 
Sinijders, R. and Wholades, K. (1996) First trinester fetal nuchal transiucency. In Ultrasound markers for fetal chromosomal defects. Snijders, R. and Nicolaides, $K$ (eds.) New York: Parthenon Publishing Group Lid, pp. 121-156.

Snijders, R. J., Sundberg, K., Holzgreve, W., Henry, G, and Nicolaides, K. H. (1999) Maternal age- and gestation-specific risk for trisomy 21. Utrasound Obster Gynecol 13: 167-170.

Spencer, $K$. (2000) Screening for trisomy 21 in twin pregnancies in the furst trimester using free beta-thCG and PAPP-A, combined with fetal nuchal translucency thickness. Prenat Diagn 20:9195.

Vamos, E., Vandenberghe, K., and Cassiman, J. J. (1997) Prenatal diagnosis in Belgium. Eur $J$ Hum Genet 5 Suppl 1: 7-13.

Wald,N. and Hackshaw,A. (2000) Tests using multiple markers. In Antenatal and neonatal screening. Wald,N., Leck, I., and (eds.) Oxford: Oxford University Press, pp. 23-57.

Wald, N. J., Cuckle, H. S., Densem, J. W. Kennard, A., and Smith, D. (1992) Matemal serum screening for Down's syndrome: the effect of routine ultrasound scan determination of gestational age and adjustment for maternal weight. BrJ Obstet Gynaecol 99: 144-149.

Wald, N. J., Cuckle, H. S., Densem, J. W., Nanchahal, K., Royston, P., Chard, T., Haddow, J. E., Knight, G. J., Palomaki, G. E., and Canick, J. A. (8-10-1988) Maternal serum screening for Down's syndrome in early pregnancy. BMJ 297: 883-887.

Wald, N. J. and Densem, J. W. (1994a) Maternal serum free alpha-human chorionic gonadotrophin levels in twin pregnancies: implications for screening for Down's syndrome. Prenat Diagn 14: 717-719.

Wald, N. J. and Densem, J. W. (1994b) Maternal serum free beta-human chorionic gonadotrophin levels in twin pregnancies: implications for screening for Down's syndrome. Prenat Diagn 14: 319-320.

Wald. N. J. Hackshaw, A. K. Hutly, W., and Kennard, A. (1996) Empirical validation of risk screening for Down's syndrome. J Med Screen 3; 185-187.

Wat, H. C., Wald, N. J, Smith, D., Kennard, A. and Densem, J. (1996) Effect of allowing for ethuic group in prenatal screening for Down's syndrome. Prenar Diagn 16:69:-698. 
Chapter 4

\section{Screening for trisomy 21 in}

\section{Flanders: a 10-years review of 40490 pregnancies screened by maternal serum}

Gyselaers WJA, Vereecken AJ, Van Herck EJH, Straetmans DPL, de Jonge ETM, Ombelet WUAM, Nijhuis JG.

Eur J Obstet Gynecol Reprod Biol 2004; 115: 185-189. 


\section{Summary}

The objective of this study was to evaluate maternal serum screening for trisomy 21 (MSS) in Flanders between 1992 and 2002.

Data of a large database on the results of MSS, nuchal translucency (NT) and pregnancy outcome were analysed retrospectively.

The performance of second trimester MSS at a maternal age $\geq 35$ years was excellent: $94.4 \%(17 / 18)$ detection rate (DR) of trisomy 21 at a false positive rate (FPR) of $22.4 \%$ (438/1956). Despite this, the proportion of patients above 35 years of age in the study population was significantly lower than in the Flemish general pregnant population $(5.5 \%$ (2227/40490) versus $8.9 \%$ $(55575 / 624433), \mathrm{p}<0.001)$.

In the population screened by MSS and NT, the DR of second trimester MSS at a $5 \%$ FPR was $44.4 \%$, which was lower than $66.6 \%$ in the population screened by MSS without NT. When 9 trisomy 21 -affected pregnancies were compared to 3265 normal pregnancies, the mean NT-MoM values were not significantly different $(1.16 \pm 0.89$ versus $1.00 \pm 0.46, p>0.05)$. Both findings comply with a sequential screening practice where second trimester MSS is only performed after a normal measurement of NT in the first trimester. 


\subsection{Introduction.}

There has been a large expansion in trisomy screening strategies over the last 15 years. Screening based on maternal age only has evolved to strategies combining maternal age with maternal serum parameters in the first or second trimester, with and without incorporation of first trimester nuchal translucency measurements (NT) by ultrasound (Cuckle, 2001a;Nicolaides, 2003). To date screening programs combining first trimester NT with first or second trimester maternal serum parameters and maternal age have been shown to produce the highest sensitivity at a false positive rate of $5 \%$ (Benn, 2003;Cuckle, 2001a; Nicolaides, 2003).

In Flanders, invasive testing is still routinely offered to pregnant women $\geq 35$ years for the antenatal detection of fetal aneuploïdy. In trisomy 21 screening programs, second trimester maternal serum parameters were introduced in 1992, followed by NT in 1999 and first trimester serum parameters in 2001. As there are no guidelines on fetal aneuploïdy screening in Flanders, a variety of screening methods is used. This study describes the application, performance and evolution of maternal serum screening (MSS) in Flanders over a ten-year period between 1992 and 2002.

\subsection{Materials and methods.}

Since 1992 maternal serum samples for second trimester trisomy screening have been analysed by the General Medical Laboratory (AML) in Antwerp, Belgium. These samples were recruited from all geographic regions in Flanders. Immunoradiometric assay was used to measure concentrations of AlfaFoetoprotein (Diagnostic Products Corporation, Los Angeles, USA) and (free) B- Human Chorionic Gonadotropin (BioSource Europe SA, Belgium). Unconjugated estriol levels were measured by radioimmunoassay (Diagnostic Systems Laboratories, Webster, USA). Pregnancy Associated Plasma ProteinA (PAPP-A) was measured by enzyme-linked immunosorbent assay (ELISA- 
DRG BioSource Belgium). The cut off level for a positive screening result was $1: 300$.

Data on pregnancy and neonatal outcome were obtained from the referring obstetricians after delivery. Once a year a list was mailed to every clinician for completion of missing data. Non responding obstetricians were contacted personally to report missing cases of trisomy 21 . Accuracy in respons rate of trisomy 21 affected pregnancies was evaluated by comparing the prevalence of trisomy 21 in the total screening population to the prevalence in Belgium as registered by Eurocat (2003). The correlation coefficient between the population with completed data and the total screening population was calculated for maternal age distribution and screen positive rates.

The results of second trimester matemal serum screening (MSS) were analysed retrospectively for different maternal age groups. Data on the distribution of maternal age in the general pregnant population from the Study Centre for Perinatal Epidemiology (SPE) were compared with the maternal age distribution in the study population. Statistical analysis was performed using Chi-square analysis.

Since 1999, AML also registered data on first trimester NT (mm) and crown rump length $(\mathrm{mm})$. A total of 2700 measurements were mailed to the Fetal Medicine Foundation for audit according to the FMF- reference range (Snijders et al., 1998). These data were used to calculate CRL-rellated Multiples of the Median (MoM) for every NT-value in our database.

The performance of second trimester MSS combined with NT was evaluated by comparing the detection rate of trisomy 21 (DR) at a fixed false positive rate (FPR) of $5 \%$ between MSS only and MSS in combination with NT. Mean NTMoM values of trisomy 21 affected pregnancies and normal pregnancies were calculated and statistically compared by t-test.

Data on the performance of first trimester MMS + NT were limited and will not be discussed further in this paper. 
Chromosomal anomalies other than trisomy 21 were not considered in this study.

\subsection{Results.}

The total number of maternal samples analysed by AML between 1992 and 2002 was 78365 . Complete follow up data were available in $51.7 \%$, leaving a total of 40490 maternal serum samples eligible for analysis.

Table 4.1. Number and percentage of first and second trimester trisomy screenings performed between 1992 and 2002 with completed data on pregnancy outcome.

\begin{tabular}{lccccccc} 
& \multicolumn{1}{c}{$\mathbf{1 9 9 2}-1998$} & $\mathbf{1 9 9 9 - 2 0 0 2}$ & \multicolumn{2}{c}{ Total } \\
\hline & $\mathbf{n}$ & $(\%)$ & $\mathbf{n}$ & $(\%)$ & & $\mathbf{n}$ & $(\%)$ \\
\hline 2nd trim MSS & 27900 & $(100)$ & 8482 & $(67)$ & 36382 & $(90)$ \\
2nd trim MSS + NT & & & 3274 & $(26)$ & 3274 & $(8)$ \\
1st trim MSS + NT & & & 834 & $(7)$ & 834 & $(2)$ \\
Total number & 27900 & $(100)$ & 12590 & $(100)$ & 40490 & $(100)$ \\
\hline
\end{tabular}

$\begin{array}{ll}\text { MSS: } & \text { Maternal serum sereening } \\ \text { NT: } & \text { Nuchal translucency in the first trimester }\end{array}$

Table I shows the numbers of first and second trimester maternal serum screening tests. A total of 116 trisomy 21 affected pregnancies were reported, indicating a prevalence of 1:675 in the population. Ninety nine trisomies 21 were found in a total of 67472 pregnancies screened by MSS only (prevalence 1:682) and 9 in 7339 pregnancies screened by MSS with NT (prevalence 1:815). Eight cases were present in the population screened by first trimester MSS and NT. The correlation coefficient between the population with completed follow up data and the total screening population was 0.99 , both for maternal age distribution and for screen positive rates. 
Table 2 shows the age distribution in the population with complete follow up data of women screened by maternal serum parameters with and without NT, in comparison to the maternal age distribution of the Flemish pregnant population, as registered by the SPE. The proportion of pregnant women $\geq 35$ years was significantly lower in the study population than in the general population $(5.5 \%$ versus $8.9 \%, \mathrm{P}<0.001$ ). In the total of 78365 pregnancies screened, 4964 were at maternal age $\geq 35$ years; this was also significantly lower than in the general population $(6.3 \%$ versus $8.9 \%, \mathrm{P}<0.001)$.

Table 4.2. Comparison of maternal age distribution in the study population versus the general pregnant population

\begin{tabular}{llllll}
$\begin{array}{l}\text { Maternal age } \\
\text { (years) }\end{array}$ & \multicolumn{1}{c}{ General population } & \multicolumn{2}{c}{ Study population ${ }^{2}$} & \multicolumn{1}{c}{ P-value } \\
\hline$<\mathbf{2 0}$ & 13113 & $(2.1)$ & 607 & $(1.5)$ & $<0.001$ \\
$\mathbf{2 0 - 2 4}$ & $102407(16.4)$ & 6559 & $(16.2)$ & $\mathrm{NS}$ \\
$\mathbf{2 5 - 2 9}$ & $271628(43.5)$ & 18990 & $(46.9)$ & $<0.001$ \\
$\mathbf{3 0 - 3 4}$ & $181710(29.1)$ & 12107 & $(29.9)$ & $<0.001$ \\
$\geq \mathbf{3 5}$ & $55575(8.9)$ & 2227 & $(5.5)$ & $<0.001$ \\
Total & $624433(100)$ & 40490 & $(100)$ & \\
\hline
\end{tabular}

1 Data from the Studycentre for Perinatal Epidemiology (SPE) in Brussels on Fleminis deliveries between 1992 and 2002

2 Study population data from the database of the General Medical Laboratory (AML) in Antwerp on all first and second trimester screcnings with complete data on pregnancy outcome, performed between 1992 and 2002.

Table 3 shows the detection rates (DR) and false positive rates (FPR) at a 1:300 risk calculation cut- off for second trimester MSS in different age categories. The overall sensitivity was $69.7 \%$ and specificity $94.5 \%$. The detection rates and false positive rates were clearly associated with maternal age, i.e. ranging from $50 \%$ and $1.5 \%$ respectively, in the maternal age category of $<20$ years to $100 \%$ and $32.1 \%$ in the maternal age category of $>37$ years. 
Table 4.3. Deicction rates for trisomy 21 and false positive rates of second trimester matcmal serum screening as a single test in different matemal age groups

\begin{tabular}{|c|c|c|c|c|c|}
\hline Maternal age & Triple & Tri & y 21 & DR & FPR \\
\hline (years) & & + & - & $(\%)$ & $(\%)$ \\
\hline$<20$ & $\mathrm{TT}+$ & 2 & 8 & & \\
\hline & TT. & 2 & 539 & 50.0 & 1.5 \\
\hline $20-24$ & TTt & 1 & 150 & & \\
\hline & TT. & 1 & 5655 & 50.0 & 2.6 \\
\hline $25-29$ & TTt & 18 & 606 & & \\
\hline & TT: & 12 & 16652 & 60.0 & 3.5 \\
\hline $30-34$ & $\mathbf{T T}+$ & 31 & 791 & & \\
\hline & TT- & 14 & 9926 & 68.9 & 7.4 \\
\hline$\geq 35$ & $\mathbf{T T}+$ & 17 & 438 & & \\
\hline & TT- & 1 & 1518 & 94.4 & 22.4 \\
\hline$\geq \mathbf{3 7}$ & $\mathbf{T T}+$ & 10 & 213 & & \\
\hline & TT. & 0 & 451 & 100 & 32.1 \\
\hline
\end{tabular}

\begin{tabular}{llllll} 
Overall & TT+ & 69 & 1993 & & \\
& TT. & 30 & 34290 & 69.7 & 5.5 \\
\hline
\end{tabular}

DR: Detection rate

FPR: False positive rate
TT+: $\quad$ Triple test positive $(\geq 1: 300)$

TT: $\quad$ Triple test negative $(<1: 300)$

Table 4 shows the performance of second trimester MSS with and without NT. At a fixed FPR of $5 \%$ the DR of MSS only was $66.6 \%$ and with inclusion of NT was $44.4 \%$. NT did not influence the performance of screening: in the population screened by maternal serum with NT, the DR at a fixed FPR of $5 \%$ by the algorithm MSS+age and by the algorithm MSS+age+NT were both 
$44.4 \%$. The mean NT-MoM values, calculated in the 9 pregnancies affected by trisomy 21 and in the 3265 normal pregnancies, were not significantly different $(1.16 \pm 0.89$ versus $1.00 \pm 0.46, \mathrm{P}>0.05)$.

Table 4.4. Performance of screening by second trimester maternal serum paraneters and matemal age with and without inclusion of NT.

\begin{tabular}{lll} 
& $\begin{array}{l}\text { Without NT } \\
(\mathbf{1 9 9 2 - 2 0 0 2})\end{array}$ & $\begin{array}{l}\text { With NT } \\
(\mathbf{1 9 9 9 - 2 0 0 2})\end{array}$ \\
\hline n tests with data on outcome & 36382 & 3274 \\
$\mathbf{n} \geq \mathbf{3 5}$ years (\% tests) & $1974(5.4)$ & $259(7.9)$ \\
n trisomy 21 & 99 & 9 \\
Detection Rate (\%) & $69 / 99(69.6)$ & $5 / 9(55.5)$ \\
\multicolumn{1}{c}{ 95\% conf interv (\%) } & {$[63.6-75.6]$} & {$[26.5-84.5]$} \\
False positive Rate (\%) & $1993 / 36283(5.5)$ & $248 / 3265(7.6)$ \\
DR at 5\% FPR (\%) & $66 / 99(66.6)$ & $4 / 9(44.4)$ \\
\multicolumn{1}{c}{ 95\% conf interv (\%) } & {$[60.0-73.2]$} & {$[8.1-80.7]$} \\
\hline
\end{tabular}

DR at 5\% FPR: Detection rate at a fixed false positive rate of $5 \%$

$95 \%$ conf interv: $95 \%$ confidence interval

\subsection{Discussion.}

This review of data on trisomy screening provides information on maternal serum screening practices in Flanders between 1992 and 2002. Despite a low overall response rate of $51.7 \%$ by Flemish obstetricians contributing to the AML database, the trisomy 21 affected pregnancies were reported adequately: the prevalence of 1:675 in our total screening population is similar to the prevalence of 1:721 registered by Eurocat in Belgium between 1992 and 2001 (2003). The correlation coefficient between the population with complete data and the total screening population was 0.99 . We therefore consider the data of our study group to be representative for the total population screened by MSS. 
The proportion of patients $\geq 35$ was significantly lower in our study population than in the general pregnant population (Table 2). We speculate that this effect is the result of primary invasive testing for the indication of maternal age only. In this strategy, maternal serum screening is not performed in pregnancies at materemal age $\geq 35$ years since they are submitted to an invasive procedure. Recently, one of the Belgian centres for Hurman Genetics reported that $35 \%$ of diagnostic invasive procedures in 2001 were performed for maternal age $\geq 35$ years, $28 \%$ for positive maternal serum screening and $20 \%$ for ultrasonic markers (Witters, 2002). Advanced maternal age was the first screening parameter used for the identification of a high-risk group for chromosomal anomalies. Wenstrom et al considered this parameter superior to maternal serum screening for the detection of fetal aneuploidy (Wenstrom et al., 1995). Today it becomes increasingly clear that maternal age alone is a poor parameter to be used in population screening for chromosomal anomalies, both for medical and economical reasons (Abramowicz et al., 1998; Wald et al., 1997). Several reports questioned the practice of routine invasive testing in the older maternal age group and recommended selective invasive testing of only those women who had a positive screening result (Audibert et al., 2002; Dommergues et al., 2001; Haddow et al., 1994). Our data corroborate this recommendation (Table 2): the sensitivity of second trimester maternal serum screening in our population was much higher at advanced maternal age compared to the younger maternal age group. This has also been reported by others (Cavalli et al., 1998; Haddow et al., 1994; Muller et al., 2002; Reynolds et al., 1993). We would have performed 1974 amniocenteses by routine invasive testing above 35 years of maternal age (Table 3). Selective invasive testing after prior MSS in this group reduced the number of amniocenteses to 455 , missing only one of the eighteen pregnancies affected by trisomy 21 . This reflects a loss of sensitivity of only $6 \%$ for a reduction of $77 \%$ of invasive procedures.

A review of screening strategies combining first trimester NT measurements and MSS reports higher detection rates for chromosomal anomalies at a $5 \%$ 
false positive rate, compared to screening by maternal serum parameters or $\mathrm{NT}$ only (Nicolaides, 2003). However, we found a very poor performance of MSS combined with NT (Table 4). The DR of trisomy 21 at a fixed FPR of $5 \%$ was only $44.4 \%$, which is much lower than $80-90 \%$ reported in literature and also lower than $66.6 \%$ by MSS without NT in our population (Nicolaides, 2003). Adding NT to the MSS algoritm did not alter the DR for trisomy 21 at all. The prevalence of trisomy 21 in the group screened with NT was lower compared to the group screened without NT. These findings can only be explained by changed characteristics of the population screened by maternal serum, after the introduction of NT as a screening parameter in Flanders. As first trimester NT measurement is performed approximately a month before the second trimester maternal serum screening, it is likely that a proportion of pregnant women were referred for invasive testing when an increased NT value was measured, without having any maternal serum screening test at all. In pregnancies with a normal NT measurement, screening was completed by collecting the second trimester maternal parameters. In this screening strategy, the laboratory would only receive blood samples of the pregnancies with normal NT values. This explains why in our study mean NT-MoM values in the trisomy 21 affected and normal pregnancies were equal, whereas several studies report mean NT-MoM values of $2-2.5$ in trisomy 21 affected pregnancies (Krantz et al., 2000; Snijders et al., 1998;Spencer et al., 2000;Zoppi et al., 2001). This also explains why the DR at a fixed FPR of $5 \%$ was only $44.4 \%$ in our population screened by MSS with NT whereas this was $66.6 \%$ in our population screened by MSS only. Two studies evaluated the impact of screening by first trimester NT measurement on the performance of second trimester MSS (Kadir and Economides, 1997; Thilaganathan et al., 1997). Both reported for second trimester MSS a DR of only $50 \%$ in the populations screened by first trimester NT, whereas this was much higher when first trimester NT screening was not performed. Our results are very simillar to these reports. 
The practice of screening in two steps by calculating risks using different parameters at different times, is called sequential screening. At the same DR, the FPR of sequential screening strategies is reported to be at least $30 \%$ higher than single step screening, using the same parameters (Hackshaw and Wald, 2001). In order to avoid invasive testing of unaffected pregnancies as much as possible, Flemish obstetricians can be advised to await the screening results of the combined risk calculation of both ultrasound and maternal serum parameters before discussing isolated NT measurement results with their patients. A shift from second trimester screening towards combined first trimester screening may be another solution (Cuckle, 2001b).

In conclusion, our data show a reduction of invasive procedures following second trimester maternal serum screening at maternal age $\geq 35$ years, in comparison to primary invasive testing. However, the fraction of the population submitted to this screening method between 1992 and 2002 is lower than expected from the maternal age distribution in the Flemish general pregnant population. After the introduction of NT as a screening parameter in Flanders, the screening performance of the second trimester maternal serum algoritm is apparently lower than before, which appears to be the result of a sequential screening practice. The general introduction of selective invasive testing for positive screening results only at advanced maternal age and of combined single step screening strategies may lead to a further reduction of invasive procedures in normal pregnancies as a result of fetal aneuploidy screening in Flanders.

\subsection{Acknowledgements.}

We wish to thank all Flemish obstetricians contributing to the continuing expansion of the database of trisomy screening in Flanders and the people from the General Medical Laboratory in Antwerp for the collection and filing of the data. 
Eurocat Website Database. Eurocat University of Ulster . 2003. Http:/hwww.eurocatulster,ac.uk.

Abramowicz,MJ., Reynolds, T., Vamos,E, and Zimmermann, R. (1998) Screening for chromosome and genetic abnormalities. In Textbook of perinatal medicine: a comprehensive guide to modern clinical perinatology. Kurjac,A. (ed.) London; New York: Parthenon Publishing Group Ltd, pp. 610-637.

Audibert, F., Mairovitz, V., and Frydman, R. (2002) [Altematives to amniocentesis for maternal age]. Gynecol Obstet Fertil 30: 562-566.

Benn, P. (8-3-2003) Improved antenatal screening for Down's syndrome. Lancet 361: 794-795.

Cavalli, P., Gnochi, E., Pennacchio, A., and Morrica, B. (1998) Dual analyte screening for fetal Down syndrome in pregnant women over 35 years of age. Prenat Diagn 18: 637-638.

Cuckle, H. (2001 a) Integrating antenatal Down's syndrome screening. Curr Opin Obstet Gynecol 13: $175-181$.

Cuckle, H. (17-11-2001b) Time for total shift to first-trimester screening for Down's syndrome. Lancet 358: 1658-1659.

Dommergues, M., Audibert, F., Benattar, C., Champagne, C., Gomel, V., and Frydman, R. (2001) Is routine amniocentesis for advanced maternal age stillindicated? Fetal Diagn Ther 16:372-377.

Hackshaw, A. K. and Wald, N. J. (2001) Inaccurate estimation of risk in second trimester serum screening for Down syndrome among women who have already had first trimester screening. Prenat Diagn 21: 741-746.

Haddow, J. E., Palomaki, G. E., Knight, G. J., Cunningham, G. C., Lustig, L. S., and Boyd, P. A. (21-4-1994) Reducing the need for amniocentesis in women 35 years of age or older with serum markers for screening. $N$ Engl J Med 330: 1114-1118.

Kadir, R. A. and Economides, D. L. (1997) The effect of nuchal translucency measurement on second-trimester biochemical screening for Down's syndrome. Ultrasound Obstet Gynecol 9: 244247.

Krantz, D. A., Hallahan, T. W., Orlandi, F., Buchanan, P., Larsen, J. W., Jr., and Macri, J. N. (2000) First-trimester Down syndrome screening using dried blood biochemistry and nuchal translucency. Obstet Gynecol 96: 207-213.

Muller, F., Forestier, F., and Dingeon, B. (2002) Second trimester trisomy 21 maternal serum marker screening. Results of a countrywide study of 854,902 patients. Prenat Diagn 22: 925-929.

Nicolaides, K. H. (2003) Screening for chromosomal defects. Uttrasound Obstet Gynecol 21: 313-321.

Reynolds, T. M., Nix, A. B., Dunstan, F. D., and Dawson, A. J. (1993) Age-specific detection and false-positive rates: an aid to counseling in Down syndrome risk screening. Obstet Gynecol 81 ; $447-450$. 
Snijders, R. J. Noble, P., Sebire, N., Souka, A., and Nicolaides, K. H. (1-8-1998) UK multicentre project on assessment of risk of trisomy 21 by maternal age and fetal nuchal-translucency thickness at 10-14 weeks of gestation. Fetall Medicine Foundation First Trimester Screening Group. Lance 35:2: 343-346.

Spencer, K., Spencer, C. E., Power, M., Moakes, A., and Nicolaides, K. H. (2000) One stop clinic for assessment of risk for fetal anomalies: a report of the first year of prospective screening for chromosomal anomallies in the first trimester. BJOG 107: 1271-1275.

Thilaganathan, B., Slack, A., and Wathen, N. C. (1997) Effect of first-trimester nuchal translucency on second-trimester maternal serum biochemical screening for Down's syndrome. Ultrusound Obstet Gynecol 10: 261-264.

Wald, N. J., Kennard, A., Hackshaw, A., and McGuire, A. (1997) Antenatal screening for Down's syndrome. J Med Screen 4: 181-246.

Wenstrom, K. D., Desai, R., Owen, J., DuBard, M. B., and Boots, L. (1995) Comparison of multiplemarker screening with amniocentesis for the detection of fetal aneuploidy in women $>$ or $=35$ years old. Am J Obstet Gynecol 173: 1287-1292.

Witters, I. Prenatal diagnosis of fetal malformations and chromosomal anomalies: a multidisciplinary approach. 7-158. 2002. Catholic University Louvain. 2002.

Zoppi, M. A., Ibba, R. M., Floris, M., and Monni, G. (2001.) Fetal nuchal translucency screening in 12495 pregnancies in Sardinia. Utrasound Obstet Gynecol 1.8: 649 651. 


\section{Chapter 5}

\section{Single-step maternal serum}

\section{screening for trisomy 21 in the era}

of combined or integrated

screening

Gyselaers WJA, Vereecken AJ, Van Herck EJH, Straetmans DPL, de Jonge ETM,

Ombeler WUAM, Nijhuis JG.

Gynecol Obster Invest 2004; 58: 221-224. 


\section{Summary}

Single step maternal serum screening (MSS) in the first (IMSS) or second (2MSS) trimester at maternal age $\geq 35$ years was evaluated in the North Belgian region Flanders, where difficulties are encountered in the general introduction of combined or integrated screening algorithms.

The fetal aneuploidy screening database of General Medical Laboratory AML in Antwerp was searched for 2MSS tests between 1992 and 1999 ( $\alpha$ Fetoprotein, $\beta$-Human Chorionic Gonadotropin ( $\beta$-HCG) and unconjugated Estriol, cut off 1:300) and for 1MSS tests between 1999 and 2003 (free $\beta$-HCG and Pregnancy Associated Plasma Protein-A, cut off 1:85).

At $\geq 35$ years, the detection rate for trisomy 21 (DR) was $93.8 \%(15 / 16)$ for 2MSS and the screen positive rate (SPR) was 24.5\% (504/2061). For 1MSS, these figures were $85.7 \%(6 / 7)$ and $17.7 \%$ (109/615) respectively. To detect one trisomy 21 , missed by MSS at $\geq 35$ years of age, an additional number of 1557 and 506 primary invasive procedures would be needed for 2MMS and 1MSS respectively.

We conclude that the performance of both single step 1MSS and 2MSS at maternal age $\geq 35$ years in Flanders is excellent, even without the combination with ultrasound parameters or integration of first and second trimester parameters. The simplicity of both methods allows to consider them valuable options for fetal aneuploidy screening at advanced maternal age, until high quality combined or integrated screening is accessible to all pregnant women in Belgium. 


\subsection{Introduction.}

In maternal serum screening (MSS) for fetal aneuploidy, concentrations of Pregnancy Associated Placental Protein - A (PAPP-A) and Free B- Human Chorionic Gonadotropin (HCG) are measured in the first trimester (1MSS); in the second trimester (2MSS), levels of $\mathrm{HCG}, \alpha$ - Fetoprotein (AFP) and unconjugated estriol ( $\left.\mathrm{E}_{3}\right)$ are used (Cuckle, 2000; Wald et al., 1997).

As the prevalence of fetal trisomies increases with maternal age, performance of different screening methods is reported to be better at advanced maternal age compared to younger women (Haddow et al., 1994; Muller et al., 2002a;Spencer, 2001). For medical and economical reasons, invasive procedures for positive screening results only ("selective invasive procedures") are recommended instead of routine invasive testing at maternal age $\geq 35$ years (Benn, 2003;Dommergues et al., 2001;Haddow et al., 1994;Hartnett et al., 2003; Rosen et al., 2002). Several reports are published on the performance of second trimester maternal serum screening (2MSS) at advanced maternal age (Gyselaers et al., 2004; Haddow et al., 1994; Muller et al., 2002b;Muller et al., 2002a). Few population studies are reported on first trimester maternal serum screening (1MSS) without nuchal translucency (NT) in the advanced maternal age group (Orlandi et al., 1997).

The integration of IMSS and 2MSS parameters into one single algorithm and/or their combination with ultrasound parameters are recommended to improve the results of screening (Benn, 2003; Cuckle, 2001;Nicolaides, 2003; Wald et al, 1999). However, several practical limitations are encountered when these combined or integrated methods are introduced in general screening programs for fetal aneuploidy (Benn, 2002). In some European countries, i.e. Belgium and the Netherlands, these limitations currently cause a poor access for pregnant women to high quality combined or integrated screening. In these countries, primary invasive testing for advanced maternal age is still practised without patient's counselling on alternatives. 
We investigated single step 1MSS and 2MSS at maternal age $\geq 35$ years in the North Belgian region Flanders, as a cheap and simple measure on the way to combined or integrated screening.

\subsection{Materials and methods.}

Since 1992, maternal serum samples for fetal aneuploidy screening have been analysed by the General Medical Laboratory (AML) in Antwerp, Belgium. These samples were recruited from all geographic regions in Flanders.

Samples for 2MSS were obtained between 1992 and 1998, which was before the introduction of first trimester screening in Flanders. Parameters for 1MSS were sampled between 1999 and 2003. Immunoradiometric assay was used to measure concentrations of AFP (Diagnostic Products Corporation, Los Angeles, USA) and (free) $\beta$ - HCG (BioSource Europe SA, Belgium) and levels of $E_{3}$

were measured by radioimmunoassay (Diagnostic Systems Laboratories, Webster, USA) (Renier et al., 1998). PAPP-A was measured by enzyme-linked immunosorbent assay (ELISA-DRG BioSource Belgium) (Haddow et al., 1998). The cut off level for a positive screening result was 1:300 for 2 MSS and 1:85 for the first trimester algorithm. These cut off values were chosen at the level of $5 \%$ screen positive rate (SPR) in the first 5000 pregnancies screened by each algorithm.

Data on pregnancy and neonatal outcome were obtained from the referring obstetricians after delivery. Once a year a list was mailed to every clinician for completion of missing data. Non responding obstetricians were contacted personally to report missing cases of trisomy 21 . The accuracy of reported cases of trisomy 21 was evaluated by comparison of this prevalence between the AML database and the Eurocat registry for Belgium in the time periods 1992 1998 and 1999-2001 (2003).

For this study, we used screen positive rate (SPR), which refers to all pregnancies submitted to screening, instead of false positive rate (FPR), which refers to the normal pregnancies only. 
The results of maternal serum screening (MSS) were analysed for the total population and for pregnant women $<$ and $\geq 35$ years of age.

In this report, we do not discuss chromosomal anomalies, other than trisomy 21 (i.e. trisomy 18), as collection and handling of detailed information on the outcome of these pregancies is still ongoing.

\subsection{Results.}

A total of 40419 2MSS tests was performed. In this population, 60 trisomy 21 affected pregnancies were present (prevalence 1:674). In a total of $70791 \mathrm{MSS}$ tests, 13 trisomy 21 affected pregnancies were found (prevalence 1:545). In the two time periods studied, the prevalence of trisomy 21 was $0.145 \%$ and $0.170 \%$ respectively in the AML database, which correlated well with the prevalence of $0.133 \%$ and $0.165 \%$ respectively in the Eurocat database.

Table 5.1. Performance of second trimester maternal serum screening (2MSS) by $\alpha \mathrm{FP}, \beta-\mathrm{HCG}$ and estriol at maternal age < and $\geq 35$ years, as registered by AML in Antwerp. Belgimm, between 1992 and 1998 (cut off $1: 300)$.

\begin{tabular}{|c|c|c|c|}
\hline $\mathbf{n}$ & 2MSS & DR & SPR \\
\hline & + & (\%) & $(\%)$ \\
\hline
\end{tabular}

\section{Trisomy 21 affected pregnancies}

$\begin{array}{lllll}\text { Overall } & 60 & 44 & 16 & 73.3 \\ <35 \text { y } & 44 & 29 & 15 & 65.9 \\ \geq 35 y & 16 & 15 & 1 & 93.8\end{array}$

\section{All pregnancies}

\begin{tabular}{|c|c|c|c|c|}
\hline Overall & 40419 & 2304 & 38115 & 5.7 \\
\hline$<35 y$ & 38358 & 1800 & 36558 & 4.9 \\
\hline$\geq 35 \mathrm{y}$ & 2061 & 504 & 1557 & 24.5 \\
\hline $\begin{array}{l}+ \\
\overline{D R}\end{array}$ & $\begin{array}{l}\text { Positive } \\
\text { Negative } \\
\text { Detection }\end{array}$ & $\begin{array}{l}\text { result } \\
\text { result } \\
\text { risomy }\end{array}$ & & \\
\hline SPR & Screen $p$ & & & \\
\hline
\end{tabular}


Table 1 shows the performance of 2 MSS for all women and for women aged < and $\geq 35$ years. The callculated risk was more than $1: 300$ for $5.7 \%$ of all women and the overall detection rate of trisomy 21 was $73.3 \%$. A total of 2061 women was aged $\geq 35$ years $(5.1 \%)$. In this group, 15 out of 16 trisomies 21 were detected by the screening algorithm (DR 93.8\%) and the SPR was $24.5 \%$ (504/2061). Compared to primary invasive procedures in women $\geq 35$ years of maternal age, selective invasive testing resulted in a reduction of $75.5 \%$ (1557/2061) of invasive procedures for a loss of DR of only $6.2 \%(1 / 16)$. In order to detect one trisomy 21 , which was missed by selective invasive testing following 2MSS, an additional number of 1557 primary invasive procedures would have been necessary.

Table 5.2. Performance of first trimester maternal serum screening (MSS) by free $\beta$-HCG and PAPP-A at maternal age < and $\geq 35$ years, as registered by AML in Antwerp, Belgium, between 1999 and 2002 (cut off $1: 85$ ).

\begin{tabular}{llll} 
n & 1MSS & DR & SPR \\
\hline+ & - & $(\%)$ & $(\%)$ \\
\hline
\end{tabular}

Trisomy 21 affected pregnancies

$\begin{array}{lllll}\text { Overall } & 13 & 8 & 5 & 61.5 \\ <\mathbf{3 5} \mathbf{y} & 6 & 2 & 4 & 33.3 \\ \geq \mathbf{3 5} \mathbf{y} & 7 & 6 & 1 & 85.7\end{array}$

\section{All pregnancies}

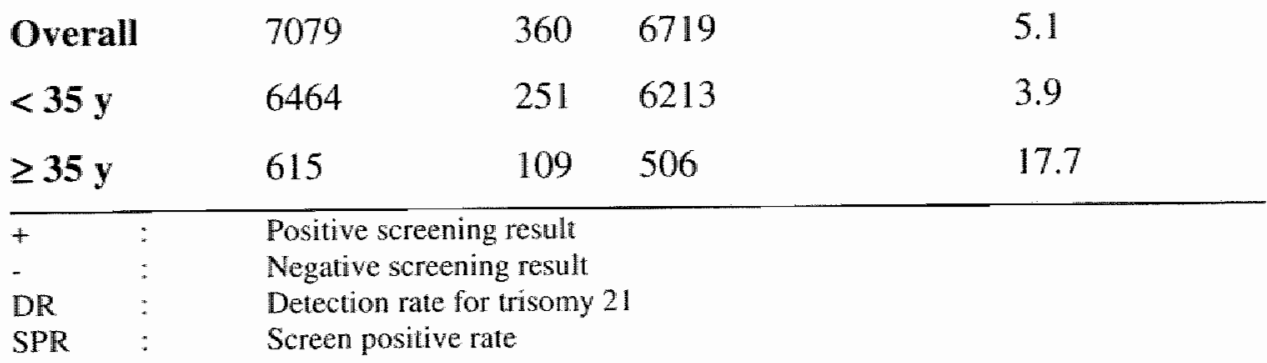


Table 2 shows the performance of 1MSS for all women and for women aged < and $\geq 35$ years. The calculated risk was $1: 85$ or more for $5.1 \%$ of women and the DR of trisomy 21 was $61.5 \%$. A totall of 615 women was aged $\geq 35$ years (8.6\%). In this group the screening algorithm detected 6 out of 7 trisomies 21 (DR $85.7 \%$ ) in a total of 109 positive screening results (SPR 17.7\%). Compared to primary invasive procedures in women $\geq 35$ years of maternal age, selective invasive testing resulted in a reduction of $82.3 \%(506 / 615)$ of invasive procedures for a loss of DR of $14.3 \%$ (1/7). In order to detect one trisomy 21 , which was missed by selective invasive testing following IMSS, an additional number of 506 primary invasive procedures would have been necessary.

In women aged $\geq 35$ years, 1 MSS detected all 7 trisomy 21 affected pregnancies at a SPR of $28.6 \%(176 / 615)$, when a cut off level of $1: 200$ was used. By changing the cut off level from 1:85 to 1:200 at maternal age $\geq 35$ years, a positive screening result was found in an additional number of 67 pregnancies, which caused an $0.9 \%(67 / 7079)$ increase of overall SPR.

\subsection{Discussion.}

This study provides information on single step maternal serum screening in Flanders, Belgium, both in the first and in the second trimester, without their combination with ultrasound parameters or the use of integrated algorithms.

The trisomy 21 affected pregnancies were reported adequately by the Flemish obstetricians, as is shown by the correlation between the AML database and the Eurocat Register of Birth Defects in Belgium for the prevalence of trisomy 21 between 1992 and 2001 (2003).

The performances of both the second and first trimester algorithms we used in this study were very similar to other reports. Wald et al. reviewed second trimester MSS in 1997 and found detection rates for trisomy 21 varying between 42 and $89 \%$ at SPR between 3 and 13.3\% (Wald et al., 1997). We found a DR of $73.3 \%$ at a SPR of $5.7 \%$ of all pregnancies. At maternal age $\geq 35$ years, these rates were respectively $93.8 \%$ and $24.5 \%$ in our study. This is also 
similar to other reports (Haddow et al., 1994; Muller et al., 2002b; Muller et al., 2002a). First trimester MSS was reported to achieve a DR for trisomy 21 varying between 60 and $85 \%$ at SPR of 5 to 25\% (Bindra et al, 2002; Haddow et al., 1998; Muller et al., 2003; Wapner et al., 2003). In our study, trisomy 21 DR was $61.5 \%$ and the overall SPR was $5.1 \%$ at a cut off of $1: 85$. For IMSS combined with nuchal translucency (NT), an increase of DR for chromosomal anomalies was reported with advancing maternal age (Krantz et al, 2000; Spencer, 2001; Wapner et al., 2003). Few data are reported on this association within the algorithm 1MSS without NT (Orlandi et al., 1997). In our study, at a cut off of 1:85, 1MSS detected $61.5 \%$ of all trisomy 21 affected pregnancies and $85.7 \%$ at $\geq 35$ years. The overall SPR was $5.1 \%$ and $17.7 \%$ for women aged $\geq 35$ years. At a cut off level of 1:200, our algorithm detected all cases of trisomy 21 at maternal age $\geq 35$ years, and the SPR differed less than $1 \%$ from the SPR at cut off of $1: 85$.

We evaluated the rate of invasive procedures by primary invasive testing al advanced maternal age versus selective invasive procedures only for positive MSS results. At maternal age $\geq 35$ years, our data indicate that selective invasive testing only for positive IMSS or 2MSS achieves a reduction of over $75 \%$ of invasive procedures compared to routine invasive testing. For first trimester chorionic villus sampling and second trimester amniocenteses, a procedure related complication rate of $0.5-1 \%$ is reported (Alfirevic et al., 2003). We would need to perform an additional number of 506 primary invasive procedures to detect one trisomy 21 missed by IMSS, which as a result would cause an expected number of 2 to 5 lost pregnancies. In the population screened by 2MSS, we would even need to perform an additional number of 1557 invasive procedures to detect one trisomy 21 missed by the algorithm, which would then lead to an expected number of 8 to 15 lost pregnancies. Our data confirm the findings of others (Dommergues et al, 2001; Haddow et al, 1994; Hartnett el al., 2003; Rosen et al., 2002). 
According to several reviews, the performance of combined or integrated screening algorithms is much better than for single step maternal serum screening (Benn, 2003; Cuckle, 2001; Nicolaides, 2003; Wald et al., 1999). In our study population, we also expect an improval of the screening performance from the combination or integration of ultrasound parameters, obtained in the first (nuchal translucency, nasal bone) or in the second trimester (genetic scan), into the maternal serum algorithms. However, several practical limitations are reported in introducing combined or integrated screening for general obstetric populations: these include scheduling of testing within relatively narrow gestational age intervals, availability of appropriately trained ultrasonographers and costs (Benn, 2002). Due to these limitations, high quality combined or integrated screening is not yet available or easily accessible for all pregnant women in many developed countries, i.e. Belgium and the Netherlands. Therefore many obstetricians currently still prefer to recommend primary invasive testing by amniocentesis or chorionic villus sampling to women $\geq 35$ years of age, meanwhile offering non invasive screening methods to younger women. In Belgium, this practice is likely to continue until training and audit of a sufficient number of ultrasonographers and National Health Insurance funding of multiple parameter screening is accomplished. As our data show, a strong reduction of primary invasive procedures and subsequent complications in Flanders may already be achieved by single step maternal serum screening for women at advanced maternal age. Selective invasive testing following 1MSS or 2MSS is easy to introduce and accessible to all pregnant women. This approach may also be the first step towards the general introduction of combined or integrated screening strategies, which are far more superior to the single step approach.

We conclude that single step maternal serum screening in the first or second trimester in Flanders achieves excellent trisomy 21 detection rates and reduces substantially the number of primary invasive procedures for women aged $\geq 35$ years. The simplicity of both methods allows to consider them valuable options 
for fetal aneuploidy screening at advanced maternal age, until combined or integrated screening is accessible to all pregnant women in Belgium.

\subsection{Acknowledgements.}

We wish to thank all Flemish obstetricians contributing to the continuing expansion of the database of trisomy screening in Flanders.

\section{References}

Eurocat Website Database. Http://www.eturocat.ulster.ac.uk. (accessed 1/10/2003).

Alfirewic, Z., Gosden, C. M., and Neilson, J. (2003) Chorion villus samplimg versus ammiocentesis for prenatal diagnosis (Cochrane review). Cochrane Library 2003.

Benn. P. (8-3*2003) Improved antenatal screening for Down's syndiome. Lancet 361: 794-795.

Benn, P. A. (2002) Advances in prenatal screening for Down syndrome: II first trimester testing. integrated testing, and luture directions. Clin Chim Acta 324:1-11.

Bindra, R., Heath, V., Liao, A., Spencer, K., and Nicolaides, K. H. (2002) One-stop clinic for assessment of risk for trisomy 21 at $11-14$ weeks: a prospective study of 15030 pregnancies. Uitrasound Obstet Gynecol 20: $219-225$.

Cuckle, H. (2000) Bhochemical screening for Down syndrome. Eur J Obster Gynecol Reprod Biol 92: 97-101.

Cukkle, H. (2001) Integrating antenatal Down's syndrome screening. Curr Opin Obster Gynecol 13: $175-181$

Dommergues, M., Audibert, F., Benattar; C., Champagne, C., Gondel, V., and Frydman, R. (2001) Is routine amniocentesis for advanced maternal age still indicated? Fetal Diagn F/her $16: 372-377$.

Gyselaers, W., Vereecken, A., Van Herck, E., Straetmans, D., Martens, G., De Jonge, E., Ombelet, W., and Nijhuis, J. (2004) Screening for trisomy 21 in Flanders: al 10-years review of 40490 pregnancies screened by maternal serum. Ear J Obste Gynecol Reprod Biol 115: 185-189.

Haddow, J. E., Palomaki, G. E., Knight, G. J., Cunninghan, G. C., Lustig, L. 5., and Boyd, P. A. (21.4 1994) Reducing the need for ammiocentesis in women 35 years of age or older with serum markers for screening. $N$ Engl I Med 330: 11:14-1118.

Haddow, J. E., Palomaki, G. E., Knight, G. J., Williams, J., Miller, W. A., and Johnson, A. (2-4. 1998) Soreening of maternal serum for fetal Down's syndrome in the first trimester. $\mathrm{N}$ Engl I Med 338: $955-961$.

Hartnett, J., Borgida, A. F., Benn, P. A., Feldman, D. M., DeRoche, M. E., and Egan, J. F. (2003) Cost analysis of Down syndrome screening in advanced matemal age. I Marenn Fetal Neomatal Med 13:80-84. 


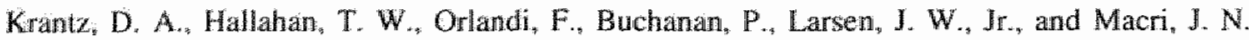
(2000) First-trimester Down syndrome screening using dried blood biochemistry and nuchal trinslucency. Obstet Gynecol 96: 207-213.

Muller, F, Benattar, C., Audibert, F., Roussel, N., Dreux, S., and Cuckle, H. (2003) Firsttrimester screning for Down syndrome in France combining fetal nuchal translucency measurenent and biochemical markers. Prenar Diagn 23: 833-836.

Mulfer, F., Forestier, F., and Dingeon, B. (2002a) Second trimester trisomy 21 matemal serum marker screening. Results of a countrywide study of 854,902 patients. Prenat Diagn 22: 925-929.

Muller, F., Thalabard, J. C., Ngo, S., and Dommergues, M. (2002b) Detection and false-positive rates of maternal serum markers for Down syndrome screening according to maternal age in women over 35 years of age. A study of the agreement of eight dedicated software packages. Prenat Diagn 22: 350-353.

Nicolaides, K. H. (2003) Screening for chromosomal defects. Ultrasound Obstet Gynecol 21: $313-321$

Orlandi, F. Damiani, G., Hallahan, T. W., Krantz, D. A., and Macri, J. N. (1997) First-trimester screening for fetal aneuploidy: biochemistry and muchal translucency. Ultrasound Obstet Gynecol 10: $3811-386$.

Renier, M. A., Vereeken, A., Van Herck, E., Straetmans, D., Ramaekers, P., and Buytaert, P. (1998) Second trimester maternal dimeric inhibin-A in the multiple-marker screening test for Down's syndrome. Hum Reprod 13: 744-748.

Rosen, D. J., Kedar, I., Amiel, A., Ben Tovim, "T., Petel, Y., Kaneti, H., Tohar, M., and Fejgin, M. D. (2002) A negative second trimester triple test and absence of specific uiltrasonographic markers may clecrease the need for genetic amniocentesis in advanced maternal age by $60 \%$. Prenat Diagn 22: $59-63$.

Spencer, K. (2001) Age related detection and false positive rates when screening for Down's symdrome in the first trimester using fetal nuchal translucency and maternal serum free betahCG and PAPP-A. BVOG 108: 1043-1046.

Wald, N. J., Kennard, A., Hackshaw, A., and McGuire, A. (1997) Antenatal screening for Down's syndrome. J Med Screen 4: 181-246.

Wald, N. J., Watt, H. C., and Hackshaw, A. K. (12-8-1999) Integrated screening for Down's syndrome on the basis of tests performed during the first and second trimesters. $N$ Engl $J$ Med $341: 461-467$.

Wapner, R., Thom, E., Simpson, J. L., Pergament, E., Silver, R., Filkins, K., Platt, L., Mahoney, M., Johnson, A., Hogge. W. A., Wilson, R. D., Mohide, P., Hershey, D., Krantz, D., Zachary, J., Snijders, R., Greene, N., Sabbagha, R., MacGregor, S., Hill, L., Gagnon, A., Hallahan, T., and Jiackson, L. (9-10-2003) Fïrst-trimester screening for trisomies 21 and 18. $N$ Engl J Med 349: $1405-1413$. 
Chapter 6

\section{Audit on nuchal translucency}

thickness measurements in

\section{Flanders, Belgium:}

a plea for methodologic

standardisation

Gyselaers WJA, Vereecken AJ, Van Herck EJH, Straetmans DPL, de Jonge ETM, Ombelet WUAM, Nijhuis JG.

Ultrasound Obstet Gynecol 2004; 24:5/1-5/5. 


\section{Summary}

Objective. To audit nuchal translucency (NT) measurements for fetal aneuploidy screening in Flanders, and to estimate the impact of small variations of NT measurement on the screening result of two first trimester screening algorithms: maternal age + NT (A), and maternal age + NT + PAPP-A + HCG (B).

Methods. We used the database of first trimester combined screening, as collected by the General Medical Laboratory AML in Antwerp, Belgium, between $1 / 1 / 2001$ and 1/4/2004. Audit was performed by establishing a deltaNT distribution curve for one Fetal Medicine Foundation (FMF) trainee and for a group of 263 other ultrasonographers, in comparison to the FMF reference values. Risks for fetal aneuploidy were calculated at cut off 1:300 for $\mathrm{A}$ and 1:150 for B. These risks were recalculated in both algorithms after modelled increase of all NT values by +0.1 or $+0.2 \mathrm{~mm}$.

Results. In a total of 592 measurements performed by the FMF trainee, the 5 th, 50 th and 95 th percentile of delta-NT measurements were at $-0.41,+0.03$ and $+0.68 \mathrm{~mm}$ respectively. These values were close to the FMF reference values. The screen positive rate (SPR) for this set of data was $4.4 \%(26 / 592)$ in both algorithms. For the 12555 measurements of the 263 other ultrasonographers, the 5 th, 50 th and 95 th percentile of delta-NT were at -0.81 , 0.14 and $+0.73 \mathrm{~mm}$ respectively, which clearly indicates underestimation of NT in the lower range. In this set of data the SPR was $3.5 \%$ for both algorithms (439/12555 for A and 436/12555 for B). Also in this group, 5\% (59/1186) of negative screening results at maternal age $\geq 35$ years in algorithm $A$, reversed to positive after modelled $0.1 \mathrm{~mm}$ increase of NT, whereas this was only in $1.2 \%$ $(134 / 11369)$ tests at $<35$ years $(p<0.0001)$. The overall increase of SPR in algorithm A after $+0.1 \mathrm{~mm}$ NT modification was $1.2 \%(152 / 12555)$, which was significantly more than $0.7 \%(86 / 12555)$ in algorithm $B(p<0.0001)$. 
Conclusion. In Flanders, a systematic underestimation of NT is found in comparison to the FMF reference range. Attempts to change these measurements according to the FMF criteria are crucial, and will mainly influence the screening results of women at advanced maternal age and of NT based algorithms without the use of other parameters. 


\subsection{Introduction.}

Nuchal translucency (NT), measured in the first trimester of pregnancy, has become the most important ultrasonic parameter for fetal aneuploidy screening today. In combination with maternal age (Snijders et al., 1999), NT can be used either as a single parameter or in combination with first or second trimester maternal serum parameters to calculate the individual's risk for chromosomal anomalies (Nicolaides, 2003; Snijders et al., 1998). Standardisation in the methodology of ultrasonic NT measurement has been shown to be mandatory for appropriate screening for chromosomal anomalies (Monni et al., 1997; Nicolaides et al., 2002). To achieve this, training and audit are recommended (Braithwaite et al, 1996; Snijders et al., 2002; Wojdemann et al., 2001).

The introduction of NT as a fetal aneuploidy screening parameter in general obstetric populations may be troubled with several practical difficulties, such as scheduling of testing within narrow gestational age intervals or the availability of appropriately trained ultrasonographers (Benn, 2002; Welch and Malone, 2003). In Flanders (North Belgium), NT was introduced as a screening parameter in 1996 and is now commonly used in fetal aneuploidy screening programs. However, few obstetricians performing NT scans comply with training and audit programs. We wondered whether and how this would influence the screening results of first trimester screening in a low risk obstetric population.

\subsection{Materials and methods.}

Between 1/1/2001 and 1/4/2004, maternal serum samples for first trimester trisomy screening have been analysed by the General Medical Laboratory (AML) in Antwerp, Belgium. These samples were recruited from all geographic regions in Flanders. All samples were accompanied with data on first trimester NT and crown rump length (CRL) in $\mathrm{mm}$. The first trimester ultrasound scans were performed by the obstetricians, referring the blood samples for analysis. 
No details were provided on the methodology of NT measurement. Only data with CRL values between 45 and $85 \mathrm{~mm}$, as recommended by the Fetal Medicine Foundation (FMF), were considered in this study (Nicolaides et al, 2002).

A total of 2700 NT/CRL measurements were mailed to FMF. From these data, CRL-related Multiples of the Median (NT-MoM) were mailed back to us, which we used to establish the FMF reference curve of gestation related NT-values. From this, NT-MoM values could be defined for every NT measurement in our database, which were used to calculate risks for fetal aneuploidy. Biochemical analysis for Pregnancy Associated Plasma Protein- A (PAPP-A) was performed using an enzyme-linked immunosorbent assay (ELISA 2397, DRG International Inc, USA). Free $\beta$-Human Chorionic Gonadotropin (HCG) was measured by radio-immunoassay (Free B-HCG IRMA KIPI00I, BioSource Europe SA, Belgium). For PAPP-A, the intra- and inter- assay coefficients of variation were less than $10 \%$ and for Free $\beta-\mathrm{HCG}$ they were less than $5 \%$ and $10 \%$ respectively. Each result was adjusted for maternal weight (de Graaf et al., 1999), multiple gestation (Spencer, 2000), smoking (de Graaf et al., 2000) and ethnicity (Hallahan et al., 2004) and was expressed as a multiple of the median (MoM). Medians and MoM values were controlled and improved each month with an exponential regression analysis using NCSS Statistical Software.

For all parameters, we used the likely hood ratio's and correlation coefficients as reported by de Graaf et al.(de Graaf et al., 1999) Risk calculations were performed using the algorithm reported by Palomaki, which can be used freely if aknowledged as such (Palomaki, 1992, Foundation for Blood Research, Box 190, Scarborough, ME 04074, USA). For every pregnant women, the risk for fetal aneuploidy was calculated in two algorithms: screening by maternal age + NT (A) and screening by maternal age $+\mathrm{NT}+$ maternal serum parameters (B). The screen positive rate (SPR) of these algorithms was calculated as the number of positive screening results over the total number of screening tests. The cut off level for positive screening was 1:300 for A (Snijders et al., 1998) and 1:150 for 
B. These cut off levels were defined at the 5\% SPR of the first 5000 samples in our database. As data collection on the outcome of the screened pregnancies is still ongoing, we did not focus on fetal aneuploidy detection rates in this study. Audit on NT measurement was performed in a similar way as the FMF approach. For every CRL-related NT value, we calculated delta -NT, defined as the difference in mm between the measured NT value and the median NT value at the same length of CRL (Spencer et al., 2003). From the FMF software for audit on NT measurement, we defined the reference 5th, 50th and 95th percentile of delta-NT at $-0.45 \mathrm{~mm}, 0 \mathrm{~mm}$ and $+0.75 \mathrm{~mm}$ respectively. All delta-NT values of our database were compared to these FMF reference values in a delta-NT distribution curve. Distribution curves were established for two groups of ultrasonographers: one ultrasonographer who was trained and audited by FMF and the others, of whom we had no further details with respect to their qualifications. In the second group, distribution curves were also established for two groups of pregnant women : those younger than 35 years of age and those aged 35 years or more.

The effect of small variations of NT measurement on the final screening results was evaluated by mathematic modelling of the NT values. In our model, every NT measurement obtained from the group of ultrasonographers was increased by 0.1 or $0.2 \mathrm{~mm}$ and the SPR of both algorithms was recalculated. The SPR shift, as caused by this modelling, was compared statistically by student t-test between women aged $\geq 35$ years and $<35$ years and between both algorithms.

\subsection{Results.}

We collected a total of 15013 maternal serum samples and NT measurements, which were obtained from 264 obstetricians, active in 45 different centres in Flanders. In this population, the mean matemal age was $29.3 \pm 4.3$ years. A total of 13559 women were aged < 35 years $(90.3 \%)$, 1454 were 35 years or more $(9.7 \%)$. The measurements of CRL ranged between 35 and $83 \mathrm{~mm}$ and the mean CRL length was $55.5 \pm 9.4 \mathrm{~mm}$. A total of $1866 \mathrm{CRL}$ measurements 
(12.4\%) were less than $45 \mathrm{~mm}$, which was the minimum length for NT measurement as recommended by FMF (Nicolaides et al., 2002). These data were excluded from the study, leaving a total of 13147 data for further analysis. A total of 592 NT/CRL measurements $(4.5 \%)$ were performed by the FMF trained ultrasonographer and 12555 by the others.

Figure 6.1. Distribution curves of 13147 NT measurements collected by AML, Antwerp Belgium, expressed as delta NT (mm). The FMF reference values are at the crossings of the horizontal and vertical dotted lines: the delta NT 5 th centile is at $-0.45 \mathrm{~mm}$, the 50 th centile is at 0 mm and the 95 th centile is at $+0.75 \mathrm{~mm}$. The distribution curves by one. FMF trainee and by a group of 263 other ultrasonographers are shown in relation to these reference values.

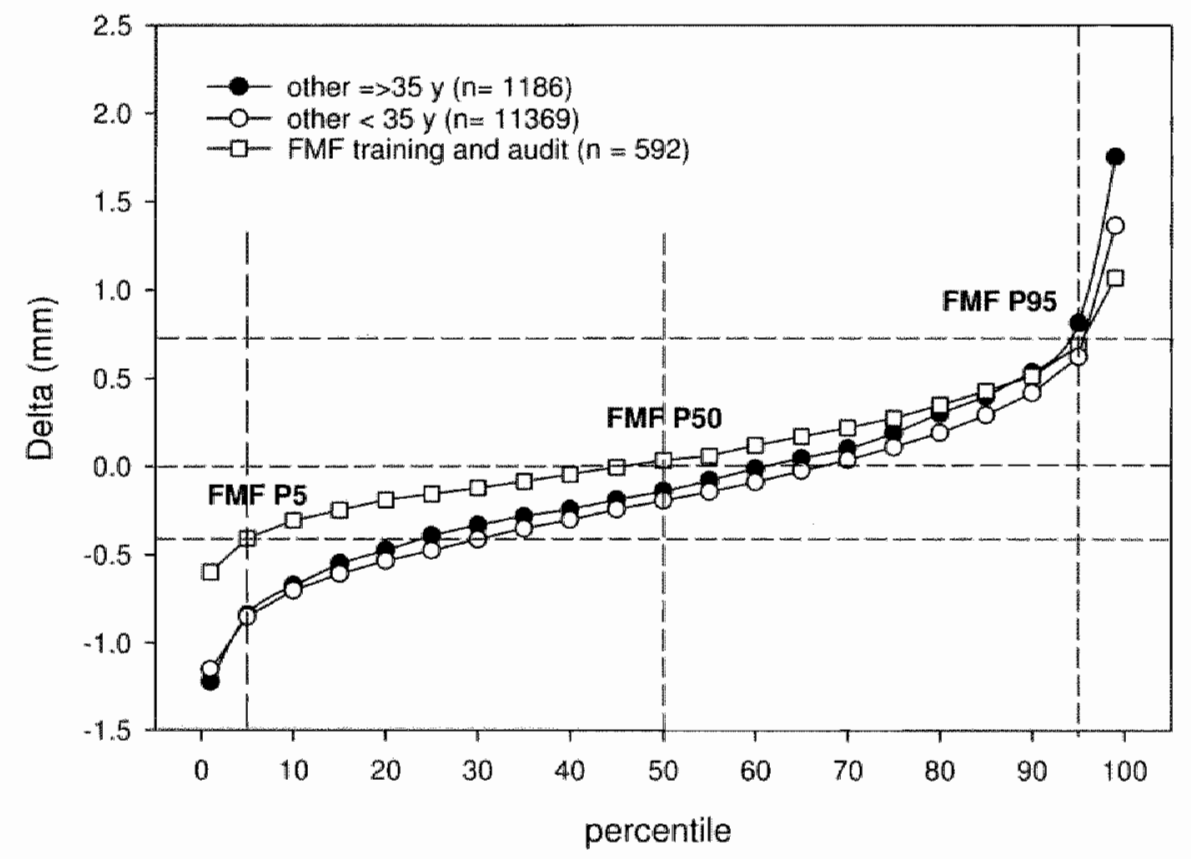

The distribution curves of delta NT, as measured by the FMF trainee and the other ultrasonographers, were clearly different (Fig 1). The 5th, 50th and 95th percentile of the 592 delta NT values from the trained ultrasonographer were at $-0.41 \mathrm{~mm},+0.03 \mathrm{~mm}$ and $+0.68 \mathrm{~mm}$. These values were very close to the FMF reference values, as is shown in Figure 1. The distribution curve of the 12555 delta NT values from the other obstetricians showed different values: the 5th, 
50th and 95th were at $-0.81 \mathrm{~mm},-0.14 \mathrm{~mm}$ and $+0.73 \mathrm{~nm}$. This distribution was similar for women $<$ and $\geq 35$ years of age (Figure 1 ).

The overall screen positive rate (SPR) for the data of the FMF trainee was $4.4 \%$ $(26 / 592)$ in both the algorithm $\mathrm{A}$ and $\mathrm{B}$. These values were $3.5 \%$ in both algorithms for the data of the other ultrasonographers (439/12555 for $A$ and $436 / 12555$ for $B$ ).

The SPR varied considerably following modelling of NT in the data from the group obstetricians, excluding the FMF trainee. The overall. SPR of algorithm A increased from $3.5 \%$ to $4.7 \%(588 / 12555)$ when the NT values increased by 0.1 $\mathrm{mm}$ and to $6.5 \%(816 / 12555)$ when the NT measurements increased by $0.2 \mathrm{~mm}$. This indicates that +0.1 or $+0.2 \mathrm{~mm}$ NT modification reversed negative screening results into positive in $1.2 \%$ and $3.0 \%$ respectively. Similarly, the overall SPR of algorithm B increased from $3.5 \%$ to $4.2 \%(522 / 12555)$ when the NT measurement increased by $0.1 \mathrm{~mm}$ and to $4.9 \%(615 / 12555)$ when the NT measurements increased by $0.2 \mathrm{~mm}$. This indicates that +0.1 or $+0.2 \mathrm{~mm} \mathrm{NT}$ modification reversed negative screening results into positive in $0.7 \%$ and $1.4 \%$ respectively. These SPR shifts were significantly higher in algorithm $A$ than in algorithm $B(p<0.0001)$.

Also for the measurements by the group of ultrasonographers, excluding the FMF trainee, Figure 2 shows that the SPR of algorithm A increased in relation to the value of delta-NT. The SPR shift following +0.1 or $+0.2 \mathrm{~mm}$ increase of NT values was also dependent on the value of delta-NT. A shift of SPR following $+0.2 \mathrm{~mm}$ modification of NT did not occur for $14.1 \%$ (1770/12555) of delta NT-values below $-0.4 \mathrm{~mm}$ (Figure 2 ). In $72.3 \%$ of delta-NT values (9077/12555) between -0.4 and $+0.4 \mathrm{~mm}, 121$ negative screening results $(1.3 \%)$ reversed to positive after increasing NT by $0.1 \mathrm{~mm}$, which is significantly less than 142 out of 1708 screening tests $(8.3 \%)$ with delta-NT values $\geq+0.4 \mathrm{~mm}(\mathrm{p}$ $<0.0001)$. 
Figure 6.2. Screen positive rates at a 1:300 cut off by the algorithm maternal age + NT in relation to delta-NT values as measured by the 263 obstetricians, except the FMF trainee. True NT values (NT), and modified NT values (NT $+0.1 \mathrm{~mm}$ or +0.2 mm) were used to calculate risks for fetal aneuploidy.

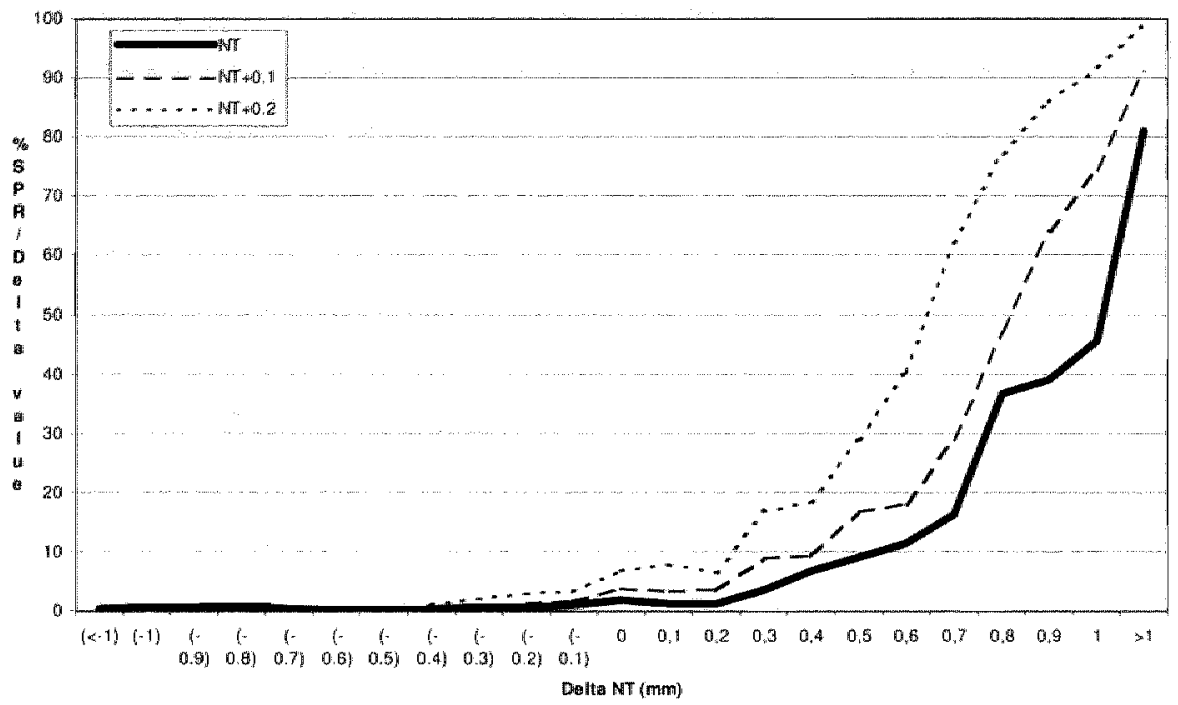

Figure 6.3. Screen positive rates at a 1:300 cut off by the algorithm maternal age + NT in the population screened by the 263 obstetricians, except the FMF trainee, in relation to maternal age. True NT values (NT), and modified NT values (NT $+0.1 \mathrm{~mm}$ or $+0.2 \mathrm{~mm}$ ) were used to calculate risks for fetal aneuploidy,

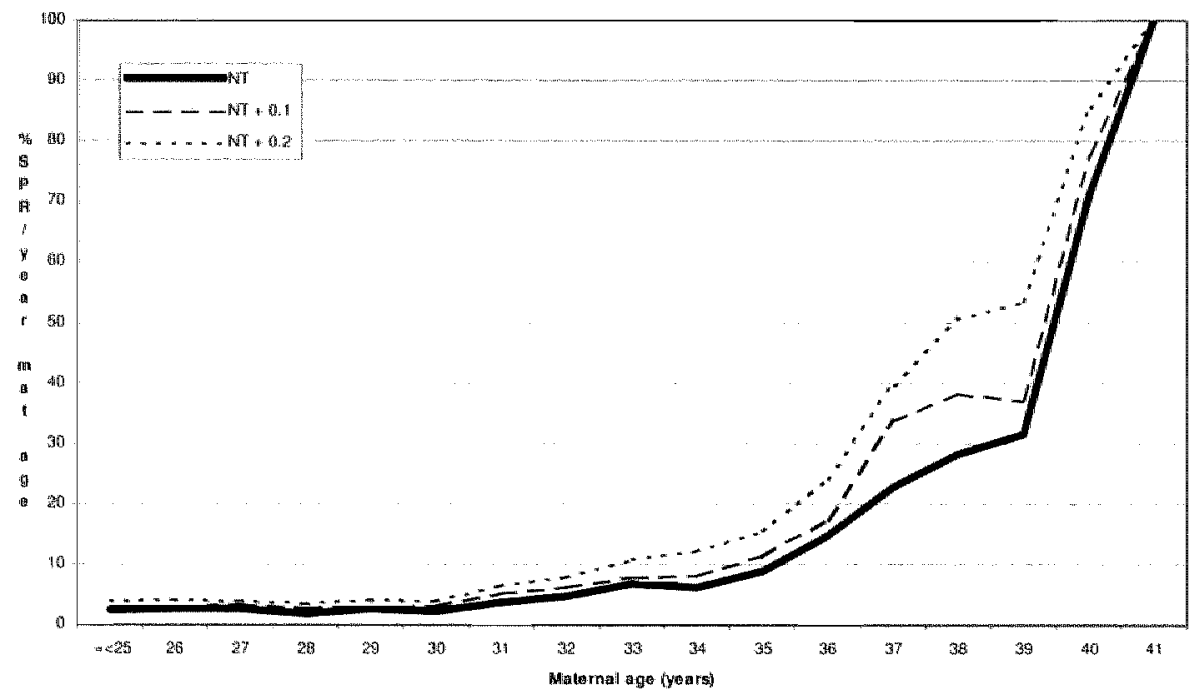


Similar results were found for the association between matemal age and SPR of algorithm A. This is shown in Figure 3. The impact of a +0.1 or $+0.2 \mathrm{~mm}$ modification of NT values on SPR was stronger with advancing maternal age. In a total of 11369 pregnancies at maternal age $<35$ years, 134 negative screening results turned positive after increasing NT by $0.1 \mathrm{~mm}(1.2 \%)$, which is significantly less than 59 out of 1186 screening tests $(5.0 \%)$ at maternal age $\geq$ 35 years $(\mathrm{p}<0.0001)$.

\subsection{Discussion.}

This study illustrates some of the difficulties to introduce standardised measurement of NT in fetal aneuploidy screening of a general obstetric population. It also focusses on the impact of (in)appropriate NT measurement on first trimester screening results.

In Flanders, North Belgium, NT was introduced as a fetal aneuploidy screening parameter in 1996. Since then, a growing number of obstetricians have implemented first trimester NT measurement into daily practice. However, a limited number of Flemish obstetricians have complied to the training and audit for standardised NT measurement, as organised by the Fetal. Medicine Foundatation (FMF) (personal communication). From Figure 1, it is clear that the Flemish obstetricians are systematically underestimating NT, as compared to the FMF reference. There may be several explanations for this. (1) Because many obstetricians did not complete the FMF training program for NT measurement, it is likely that their ultrasound examinations do not comply with the recommended standardisation for image size, caliper position, contrast and fetal position (Nicolaides et al., 2002). This is illustrated in our database by the $12.4 \%$ of NT measurements performed at non standardised CRL values, which

were excluded from our study. (2) The use of appropriate equipment is mandatory to perform decent ultrasound examinations and NT measurements (Wald et al, 2003). From our data it is totally unclear how the NT measurements were obtained (abdominally or vaginally) and which ultrasound 
scanners and/or probes were used. (3) Many obstetricians perform obstetric ultrasound as an activity during routine antenatal visits at the outpatient clinics. It is possible that this strategy may cause difficulties to allocate a decent time interval for appropriate NT measurements (Wald et al., 2003). (4) There may be an attitude of obstetric health care workers to reassure pregnant women on a normal course of pregnancy, which can influence the method of NT measurement in non standardised conditions. A systematic underestimation of the true NT values may result from this.

Two different approaches are suggested to reduce the impact of NT underestimation on the results of fetal aneuploidy screening: (1) training and audit on NT measurement according to the FMF criteria (Braithwaite et al., 1996; Nicolaides et al., 2002;Pajkrt et al., 2000;Snijders et al., 2002; Wojdemann et al., 2001), and (2) the use of centre- or sonographer specific reference values into risk calculation algorithms (Crossley et al., 2002; Wald et al., 2003; Welch and Malone, 2003).

Figure I. shows the effect of training on NT measurement: the distribution curve of the FMF trainee shows values which are very close to the FMF reference values and which are larger than the measurements of the other obstetricians. It has been reported many times that training and audit on NT measurement reduces the inter-and intra observer variation and enhances the possibility to achieve measurements within the recommended reference range (Nicolaicles et al., 2002; Pajkrt et al., 2000). It has also been reported that standardised NT measurements improve the value of NT as a fetal aneuploidy screening parameter, because higher detection rates for fetal chromosomal anomalies are achieved after appropriate training (Monni et al,, 1997). Because collection of data on outcome of the screened pregnancies in our database is still ongoing, we cannot yet draw definite conclusions on the fetal aneuploidy detection rates. However, our data allow to investigate the impact of (un)standardised NT measurements on the screening results of first trimester algorithms, which is discussed in this paper. 
As our calculations show, underestimation of NT is of much more importance for the screening result when the NT values are in the higher range, compared to measurements in the lower range (Fig 2). Significantly more screening results convert from negative to positive after artificial $+0.1 \mathrm{~mm}$ increase of NT, when the initial delta-NT is $+0.4 \mathrm{~mm}$ or more in comparison to lower delta-NT values $(8.31 \%$ versus $1.33 \%$ ). As is shown in Figure 1, the delta $-\mathrm{NT}$ values above $+0.4 \mathrm{~mm}$, as measured by the Flemish obstetricians, are close to the curve of the FMF trainee and the delta NT 95th centile is also very close to the FMF reference value. This indicates that the Flemish obstetricians are measuring these critical NT values accurately. However, as is shown in Figure 3, the screening result is also strongly related to maternal age: significantly more screening results convert from negative to positive after artificial $+0.1 \mathrm{~mm}$ increase of NT at maternal age $\geq 35$ years in comparison to screening at $<35$ years $(4.98 \%$ versus $1.18 \%$ ). As is illustrated in Figure 1 , the delta-NT distribution curves by the Flemish obstetricians are similar for women $\geq$ and $<$ 35 years and show an equal underestimation of NT, mainly in the lower range. This indicates that for women at advanced maternal age, a higher number of negative screening results may be found than expected by using FMF compatible measurements. Because of this, these women may decide not to proceed to invasive testing, which puts them at higher risk for failure to detect a fetal aneuploidy antenatally.

An alternative approach to resolve the problem of NT underestimation in Flanders, is the introduction of centre- or performer- specific reference values into the screening algorithms (Crossley ef al., 2002; Wald et al., 2003). In our population, a total of 13147 NT measurements were performed by 264 different ultrasonographers from 45 different centres, which indicates a mean of 50 measurements per individual or 292 measurements per centre. This number currently is too low to establish a valuable reference curve for every single performer but should be sufficient to calculate centre specific medians. However, the use of centre specific curves in our screening algorithm should 
not withold ultrasonographers from further training and audit on NT measurement, in order to diminish large variations in NT MoM values or drifts of NT medians over longer periods of time.

Although the value of NT as a fetal aneuploidy screening parameter is well established, our data illustrate some vulnerability of NT implementation into screening algorithms. Even after appropriate training, the intra- and interobserver variability of NT measurement is reported to be $<0.5$ and $<0.6$ $\mathrm{mm}$ respectively for 95\% of measurements (Pajkrt et al., 2000; Pandya et al., 1995). Studies on the reproducibility of NT measurement show that the intraand interobserver variability is $\geq 0.1 \mathrm{~mm}$ in at least $70 \%$ of NT measurements (Newey et al., 2003; Pandya et al., 1995). For most ultrasound scanners, $0.1 \mathrm{~mm}$ is the smallest possible discriminative measurement of NT. Our data show that in algorithm $A$ up to $5 \%$ of pregnancies at maternal age $\geq 35$ years may already reverse from negative to positive after $+0.1 \mathrm{~mm}$ modification of NT. As is shown in our calculations, the overall fraction of negative screening results converting into positive after $+0.1 \mathrm{~mm}$ increase of NT measurements, is significantly smaller in the algorithm with maternal serum parameters (B) than without (A) $(0.7 \%$ versus $1.2 \%)$. Higher detection rates and lower screen positive rates are reported for multiple parameter screening algorithms in comparison to single or dual parameter algorithms (Nicolaides, 2003; Welch and Malone, 2003). We think the impact of small variations of NT measurements on the final screening result for pregnancies at advanced maternal age, may be another reason to use NT only in combination with other parameters, either derived from maternal serum or by ultrasound.

At cut off 1:300 for algorithm A, the overall SPR is $4.4 \%$ for the data of the FMF trained ultrasonographer. This figure is very close to $5 \%$ and comparable to the SPR values of several other population studies using the same algorithm at the same cut off value (Snijders et al., 1998)(Nicolaides, 2003; Welch and Mallone, 2003). The SPR of algorithm $B$ at cut off 1:150 is also $4.4 \%$. For the data of the other obstetricians, the SPR of $3.5 \%$ in both algorithms is much 
lower: there is no doubt that this is related to the underestimation of NT measurements (Figure 1).

In this study, likelihood ratio's for fetal aneuploidy were derived from NT MoM values in our algorithms and not from delta-NT values, as is recommended elsewhere (Spencer er al., 2003). Therefore, it should be investigated whether our findings also account for algorithms based on delta-NT.

In conclusion, this reports describes the underestimation of NT in comparison to the FMF reference range in Flanders, North Belgium. This can be changed by FMF training and audit. The underestimation of NT is mainly relevant to the screening results of women at advanced maternal age and is less important when other screening parameters are included into NT based screening algorithms.

\subsection{Acknowledgment.}

We wish to thank all Flemish obstetricians for their efforts to provide all relevant information to the AML database on fetal aneuploidy screening and Prof. Dr. Patrick Willems from Gendia, Antwerp, for his valuable comments. We also acknowledge the Fetal Medicine Foundation for their kind help in obtaining the FMF reference data.

\section{References}

Benn, P. A. (2002) Advances in prenatal screening for Down syndrome: Il first trimester" testing. integrated testing, and future directions. Clin Chim Acta 324: $1-11$.

Braithwaite, J. M., Kadir, R. A., Pepera, T. A., Morris, R. W., Thompson, P. J and Economides, D. L. (1996) Nuchall translucency measurement: training of potential examiners. Uhrasound Obster Gynecol 8: 192-195.

Crossley, J. A., Aitken, D. A., Caumeron, A. D., McBride, E., and Connor, J. M. (2002) Combined whrasound and bjochemical screening for Down"s syndrome in the first trimester: a Scottish multicentre study. BJOG 109: 667-676.

de Graaf, I. M., Cuckle, H. S., Pajkrt, E., Leschot, N. J., Bleker, O. P., and van Lith, J. M. (2000) Co-variables in first trimester maternal serum screening. Prenar Diagn 20: 186-189. 
de Graaf, I. M., Pajkrt, E., Bilardo, C. M. Leschot, N. J. Cuckle, H. S., and van Lith, J. M. (1999) Early pregnancy screening for fetal aneuploidy with serum markers and nuchal translucency. Prevat Diagn 19: 458-462.

Hallaham, T., Krantz, D., Buchanan, P., Larsen, J. W., and Macri, J. Effect of ethnicity of firsttrimester Down Syndrome screening. hitp:/genetics faseb.org/genetics/ashg00/2365, hum, (accessed 20-5/2004).

Monni, G., Zoppi, M. A., lbba, R. M., and Floris, M. (29-11-1997) Fetal nuchal translucency test for Down"s syndrome. Lancet 350: 1631-1632.

Newey, V. R., Nassiri, D. K., Bhide, A., and Thilaganathan, B. (2003) Nuchal translucency thickness measurement: repeatability using a virtual ultrasound scanner. Utrasound Obstet Gynecol 21: 596-601.

Nicolaides, K. H. (2003) Screening for chromosomal defects. Ultrasound Obstet Gynecol 21: $313-321$.

Nicolaides, K. H. Heath, V., and Cicero, S. (2002) Increased fetal nuchal translucency at 11-14 wecks. Prenat Diagn 22: 308-315.

Pajkrt, E., Mol. B. W., Boer, K., Drogtrop, A. P., Bossuyt, P. M., and Bilardo , C. M. (2000) Intraand interoperator repeatability of the nuchal translucency measurement. Uitrasound Obstet Gynecol 15:297-301.

Pandya, P. P., Altman, D. G., Brizot, M. L., Pettersen, H., and Nicolaides, K. H. (1995) Repeatability of measurement of fetal nuchal translucency thickness. Ultrasound Obstet Gynecol 5: $334-337$.

Snijders, R. J., Noble, P., Sebire, N., Souka, A., and Nicolaides, K. H. (1-8-1998) UK multicentre project on assessment of risk of trisomy 21 by maternal age and fetal nuchal-translucency thickness at 10-14 weeks of gestation. Fetal Medicine Foundation First Trimester Screening Group. Lancet 352: 343-346.

Snijders, R. J., Sundherg, K., Holzgreve, W., Henry, G., and Nicolaides, K. H. (1999) Maternal age- and gestation-specific risk for trisomy 21. Ultrasound Obstet Gynecol 13: 167-170.

Snijders, R. J., Thom, E. A., Zachary, J. M., Platt, L. D., Greene, N., Jackson, L. G., Sabbagha, R. E, Filkins, K., Silver, R. K. Hogge, W. A., Ginsberg, N. A., Beverly, S., Morgan, P., Blum, K., Chilis, P.. Hill, L. M., Hecker, J., and Wapner, R. J. (2002) First-trimester trisomy screening: nuchal translucency measurement training and quality assurance to correct and unify technique. Utrowsound Obstet Gynecol 19: 353-359.

Spencer, K. (2000) Screening for trisomy 21 in twin pregnancies in the first trimester using free beta-hCG and PAPP-A, combined with fetal nuchal translucency thickness. Prenat Diagn 20:911 95.

Spencer, K., Bindran, R., Nix, A. B., Heath, V., and Nicolaides, K. H. (2003) Delta-NT or NT MoM: which is the most appropriate method for calculating accurate patient-specific risks for trisomy 21 in the first trimester? Ulrasound Obstef Gynecol 22: 142-148. 
Wald, N. J., Rodeck, C., Hackshaw, A. K., Walters, J., Chitty, L., and Mackinson, A. M. (2003) First and second trimester antenatal screening for Down's syndrome: the results of the Serum, Urine and Ultrasound Screening Study (SURUSS). JMed Screen 110: 56-104.

Welch, K. K. and Malone, F. D. (2003) Nuchal translucency-based screening. Clin Obstet Gynecol 46: 909-922.

Wojdemann, K. R., Christiansen, M., Sundberg, K., Larsen, S. O., Shalmi, A., and Tabor, A. (2001) Quality assessment in prospective nuchal translucency screening for Down syndrome. Ultrasound Obstet Gynecol 18: 641-644. 
Chapter 7

\section{First trimester ultrasound}

\section{screening in Flanders, Belgium:}

- Survey on the future

organisation

- The program for training and quality control

Adapted from:

Gyselaers W, De Catte L, Witters I, de Jonge E, Ombelet W, Nijhuis J. Survey on the future organisation of first trimester altrasound screening in Flanders, 


\section{Summary}

The chief-obstetricians of the 75 maternity clinics in Flanders were asked about their opinion on the future organisation of first trimester ultrasound screening (1USS): a total of $67(89.3 \%)$ considered IUSS a routine antenatal service, to be available in every obstetric clinic, 57 (76\%) felt a need for ultrasound training into their own department and $61(81.3 \%)$ agreed for external audit and quality control on screening performance.

Following this, the most important Centres for Obstetric Ultrasound in Flanders initiated a training program for ultrasound screening techniques according to the Fetal Medicine Foundation criteria. In a 6-months period, over 200 obstetricians and junior staff members have participated in this program, which totals approximately a third of all obstetricians-gynaecologists in the country.

In the near future, the impact of this training program will be evaluated. 


\subsection{Survey on the future organisation of first trimester ultrasound screening in Flanders, Belgium.}

Nuchal translucency (NT) and other ultrasound markers have become important parameters for fetal aneuploidy screening (Nicolaides, 2003). Several difficulties are reported when ultrasound parameters are introduced in population screening for fetal aneuploidy, such as scheduling of scanning within relatively narrow gestational age intervals and the availability of appropriately trained ultrasonographers for NT measurement (Benn, 2002). Centralisation of ultrasound examinations in third level expert centres has been proposed as a measure to guarantee high quality NT screening (Egan et al., 2002). In this system however, pregnant women may have poor access to appropriate screening modalities (Welch and Malone, 2003; Whittle, 2001).

Today in Flanders, North Belgium, there are no guidelines on screening for fetal aneuploidy. We asked the chief-obstetricians of all 75 maternity clinics in Flanders about their opinion on the future organisation of first trimester ultrasound screening (1USS).

A questionnaire was sent, addressing some key-points of ultrasound screening in three questions:

1. Do you think in your own department IUSS should be available as a routine antenatal service for every pregnant woman requesting for screening?

2. Do you think training on methodology of IUSS is desirable in your department?

3. Do you agree to have an independent, external audit on the quality of IUSS in your department?

The results are enlisted in Table 1. The response rate was $92.0 \%(69 / 75)$. Of the responders, all except two centers $(67 / 69,97.1 \%)$ considered lUSS a routine antenatal service which should be available in every obstetric clinic. Of these, $82.6 \%(57 / 69)$ felt a need for training on IUSS methodology and $88.4 \%(61 / 69)$ agreed to have external quality control. 
Table 7.1. Results of the answers to the questionnaire on firsti trimester ultriasound screening, sent to the chief-obstetricians of the 75 antenatal clinics in Flanders.

\begin{tabular}{llll}
\hline & Yes & No & No answer \\
\hline In-house provision of care & $\mathbf{n}(\%)$ & $\mathbf{n}(\%)$ & $\mathbf{n}(\%)$ \\
Training on methodology & $67(89.3)$ & $2(2.7)$ & $6(8.0)$ \\
Audit and quality control & $57(76.0)$ & $12(16.0)$ & $6(8.0)$ \\
\hline
\end{tabular}

The results from this questionnaire clearly show that the majority of the Flemish chief-obstericians consider IUSS as an integral part of routine antenatal care. Today, the Belgian National Institute of Health Care Insurance (RIZIV) refunds one obstetric ultrasound scan per gestational trimester for every pregnant women. In Flanders, North Belgium, over $95 \%$ of routine antenatal care is provided by obstetricians, who also perform most of the ultrasound scanning (Kind en Gezin, 2003). The provision of IUSS in every obstetric clinic in Flanders would offer pregnant women an easy access to fetal aneuploidy screening by ultrasound, without the need to increase the number of ultrasound scans.

The performance of NT-based screening has been reported to improve after appropriate training and adherence to a standard technique (Monni et al., 1997), but also by application of a center- or performer-specific reference range for NT measurement (Wald et al,, 2003). Even when performer-specific reference ranges are used, training on $\mathrm{NT}$ measurement is still needed to reduce as much as possible the inter- and intra-observer variation and deviations from the median (Pajkrt et al., 2000). In screening, a narrow deviation from the median in the affected and unaffected populations enhances the performance of the parameter used (Cuckle and Wald, 2000). In order to guarantee high quality 
screening, the Flemish obstetricians comply to further training in methodologic standardisation of ultrasound scanning and agree for quality control through a system of external audit. These data should encourage the leading centres for obstetric ultrasound in the country to organise training sessions on ultrasound screening for all antenatal clinics in Flanders and the Belgian Government of Health Care to stimulate this process of quality achievement and control.

\subsection{The program for first trimester ultrasound training and quality control in Flanders, Belgium.}

Following the results of this survey, a program was organised in Flanders towards training and audit on first trimester ultrasound screening techniques, according to the criteria of the Fetal Medicine Foundation (FMF) (Nicolaides et al., 2002). This program was a combined initiative of the FMF and some of the most important organisations for Ultrasonography and Obstetrics in the country, such as VENEB (Vereniging van Nederlandstalige Echografisten van België), VVOG (Vlaamse Vereniging van Obstetrici en Gynecologen) and LAE (Laatavond Echo's). The program started at a symposium, chaired by Professor K. Nicolaides. Following this, a total of 8 training courses were organised in different locations throughout the country. Every course focussed on the practical aspects of first trimester ultrasound screening, both in theory and in hands-on training sessions. The companies, distributing ultrasound equipment in Belgium, presented in these sessions some of their ultrasound scanners (medium range capacity) for live demonstration of the FMF scanning techniques. The tutors of these hands-on sessions were all FMF-trainees, active in the Universities and/or the Centres for Obstetric Ultrasound in the country. The names of the tutors were enlisted at the official FMF website, except for two persons who stepped out of the FMF audit program a few years after their certification. At the end of each course, the contents of the FMF book "The 1114 weeks scan" were discussed to enable answering the Multiple Choice Questionnaire, needed for the FMF certification process 
(Hitp://www.fetalmedicine.com). As such, it was intended to guide as much participants into the FMF certification process as possible. Those participants who abstained from certification, were encouraged to collaborate with experienced ultrasonographers or to use specific software for (1) quality control, (2) the application of performer specific NT medians or (3) alternative screening methods such as integrated maternal serum screening (Http://www.Ismalpha.co.uk).

Between November 2004 and May 2005, over 200 obstetricians and junior staff members participated in these training courses, which approximates a third of the total of 620 obstetricians and juniors, currently active in Flanders.

In the near future, the impact of these training sessions will be evaluated: the number of FMF accredited ultrasonographers will be counted and shifts of the NT distribution curves by individual ultrasonographers will be evaluated, as reported elsewhere (Gyselaers et al., 2004). A new survey will be organised to evaluate the application and nature of systems towards audit and quality control of ultrasound screening performance.

The process of ultrasound training and audit was organised without logistic or financial support of the Belgian Government or prospects towards recognition or rewarding of ultrasonographers, complying to audit and quality control.

\section{References}

Bemn, P. A. (2002) Advances in prenatal screening for Down syndrome: Il first trimester testing. integrated testing, and future directions. Chin Chim Acta 324: 1-11.

Cuckle,H. and Wald,N. (2000) Priciples of screening: tests using single markers. In Antenatal \& Neonatal screening. Wald,N. and Leck,I. (eds.) Oxford: Oxford University Press, pp. 3-22.

Egan, J. F., Kaminsky, L. M., DeRoche, M. E., Barsoom, M. J., Borgida, A. F., and Benn, P. A. (2002) Antenatal Down syndrome screening in the United States in 2001: a survey of maternalfetal medicine specialists. Am J Obstef Gynecol 187: 1230-1234.

Gyselaers, W., Vereecken, A., Van Herck, E., Straetmans, D., De Jonge, E., Ombelet, W., and Nijhuis, J. (2004) Audit on nuchal translucency thickness measurements in Flanders, Belgium: at plea for methodological standardization. Ulrasound Obstet Gynecol 24: $511-515$. 
Kind en Gezin. The child in Flanders, 2002. Http:/www kindengezin be (2003).

Monni, G, Zoppi, M. A., Ibba, R. M. and Floris, M. (29-11-1997) Fetal nuchal translucency test for Down's syndrome. Lancet 350: $1631 \cdot 1632$.

Nicolatides, K. H. (2003) Screening for chromosomal defects. Uhrasound Obstef Gynecol 21 : 313-321.

Nicolaides, K. H., Heath, $V$. and Cicero, S. (2002) Increased fetal nuchal translucency at $11-14$ weeks. Prenat Diagn 22: 308-3:15.

Pajkrt, E., Mol, B. W., Boer, K., Drogtrop, A. P., Bossuyt, P. M., and Bilardo, C. M. (2000) Intraand interoperator repeatability of the nuchal translucency measurement. Ultrasound Obstet Gynecol 15: 297-301.

Wald, N. J., Rodeck, C., Hackshaw, A. K., Walters, J., Chitty, L., and Mackinson, A. M. (2003) First and second trimester antenatal screening for Down's syndrome: the results of the Serum, Urine and Ultrasound Screening Study (SURUSS). J Med Screen 10: 56 104.

Welch, K. K. and Malone, F. D. (2003) Nuchal translucency-based screening. Clin Obstet Gynecol 46: 909-922.

Whittle, M. (2001) Down's syndrome sereening: where to now? BJOG 108: 559-561. 
Chapter 8

\section{Population screening for fetal} trisomy 21: easy access to screening should be balanced against a uniform ultrasound protocol

Gyselaers WJA, Vereecken AJ, Van Herck EJH, Straetmans DPL, de Jonge ETM, Ombelel WUAM, Nijhuis $/ G$. Prenatal Diagnosis 2005 (in pres:). 


\section{Summary}

We evaluated the performance of a first trimester combined fetal aneuploidy screening algorithm using Pregnancy Associated Plasma Protein-A, free $\beta$ Human Chorionic Gonadotropin and fetal nuchal translucency (NT), with a documented underestimation of NT compared to the Fetal Medicine Foundation (FMF) reference range.

The proportion of first trimester screening tests in the total of serum screening tests performed by Algemeen Medisch Laboratorium (AML) in Antwerp, Belgium, increased from $1.3 \%(125 / 9424)$ in 2000 to $53.1 \%(6577 / 12377)$ in 2003. Only $11.4 \%(1514 / 13267)$ of NT measurements were performed according to FMF criteria. NT- Multiples of the Median (MoM), relative to the FMF reference range, were used for risk calculations.

The $80.8 \%(21 / 26)$ trisomy 21 (T21) detection rate (DR) at cut off 1:300 resulted from maternal serum screening. NT measurements did not add to this DR, but reduced the false positive rate from $16.8 \%(2212 / 13181)$ to $8.6 \%$ (1130/13181). Only $23.8 \%(5 / 21)$ of T21 detections were by FMF-trainees.

Easy access to screening and maternal serum parameters accounted for the majority of T21 detections in our first trimester combined screening program. 


\subsection{Introduction.}

In Flanders, North Belgium, over $95 \%$ of antenatal care is provided by private obstetricians, who deal with fetal aneuploidy screening and most of the obstetric ultrasound scanning (http://www.kindengezin.be). First trimester nuchal translucency (NT) thickness measurement for fetal aneuploidy screening was introduced in 1996. In combined screening algorithms, NT was first combined with second trimester maternal serum parameters, and a few years later also with first trimester maternal serum parameters (Gyselaers et al., 2004b). We recently reported our audit on NT measurements from a group of 264 Flemish obstetricians: compared to the Fetal Medicine Foundation (FMF) reference range, a systematic underestimation of NT values was found (Gyselaers et al., 2004a). Failure to comply with the guidelines for NT measurement, as recommended by FMF (Nicolaides et al., 2002), was considered to be the most likely explanation for this observation. In this paper, we discuss the introduction of our first trimester fetal aneuploidy sereening program, which combines NT with maternal serum concentrations of Pregnancy Associated Plasma Protein A (PAPP-A) and free $\beta$ - Human Chorionic Gonadotropin (FB-HCG), and we evaluate the influence of these underestimated NT-values on the screening results. The performance of our screening program is compared to published population studies on first trimester combined screening.

\subsection{Methods.}

Between 1/1/2001 and 30/4/2004, maternal serum samples for first and second trimester fetal aneuploidy screening have been analysed by the General Medical Laboratory (AML) in Antwerp, Belgium. These samples were recruited from 264 obstetricians, active in 35 centres located in all geographic regions of Flanders. All serum samples, sent for combined screening, were accompanied with data on first trimester NT and crown rump length (CRL) in $\mathrm{mm}$. The obstetricians, referring the blood samples for analysis, performed the first trimester ultrasound scans. The FMF-accredited ultrasonographers were 
identified at the official FMF-website (http:/www fetalmedicine.com/fcompetence.htm). From our audit, it was concluded that the NT measurements from the FMF-trainees were according to the FMF criteria. As no information was available on the ultrasound qualifications of the other ultrasonographers, their NT measurements were defined "unspecified".

We calculated the yearly number of all maternal serum tests performed by AML in the time period 2000-2003, as the sum of all second trimester maternal serum screening tests, with and without NT, and the first trimester combined screening tests.

A total of 2700 NT/CRL measurements were mailed to FMF. From these data, CRL-related Multiples of the Median (NT-MoM), relative to the FMF reference range, were mailed back to us. From this, FMF specific NT-MoM values could be defined for every NT measurement in our database, which were used to calculate risks for fetal aneuploidy. Biochemical analysis for Pregnancy Associated Plasma Protein- A (PAPP-A) was performed using an enzymelinked immunosorbent assay (ELISA 2397, DRG International Inc, Mountainside New Jersey,USA). Free $\beta$-Human Chorionic Gionadotropin (HCG) was measured by radioimmunoassay (Free $\beta$-HCG IRMA K1P1001, BioSource Europe SA, 1440 Nivelles, Belgium). For PAPP-A, the intra- and inter-assay coefficients of variation were less than $10 \%$, and for Free $\beta-\mathrm{HCG}$ they were less than $5 \%$ and $10 \%$ respectively. Each result was adjusted for maternal weight (de Graaf et al., 1999), multiple gestation (Spencer, 2000), smoking (de Graaf et al., 2000) and ethnicity (Hallahan er al., 2004) and was expressed as a multiple of the median (MoM). Medians and MoM values were controlled and improved each month with an exponential regression analysis using NCSS Statistical Software (NCSS, 329 North 1000 East-Kaysville, Utah 84037, USA).

For all parameters, we used the likely hood ratio's and correlation coefficients as reported by de Graaf et al. (de Graaf et al., 1999). Risk calculations were performed using the algorithm reported by Palomaki, which can be used freely 
if acknowledged as such (Palomaki, 1992, Foundation for Blood Research, Box 190, Scarborough, ME 04074, USA). We validated our risk calculation algorithm by logarithmic correlation of the calculated risks with the observed risks in our population (Onda et al., 1998; Wald et al., 1997).

At least once a year, obstetricians reported the outcome of the screened pregnancies by mail. Non-responding obstetricians were contacted personally to collect missing data on chromosomal anomalies. The data were grouped in trisomy 21 (T21)-affected pregnancies, pregnancies affected by fetal chromosomal anomalies different from $\mathrm{T} 21$, pregnancies with spontaneous fetal loss and unaffected pregnancies. For this study, only the T21-affected and unaffected pregnancies were considered.

The validity of our registration was evaluated by comparing the prevalence of $\mathrm{T} 21$ at term, as expected from our observations, with the prevalence of $\mathrm{T} 21$, as expected from the maternal age distribution in our population and the data on maternal age and gestation related prevalence as registered in the British National Down Syndrome Cytogenetic Register (Morris et al., 2002b);(Morris et al., 1999; Morris et al., 2002a).

In the group of affected pregnancies, we defined the T21 detection rate (DR) at 12 week risk cut off values 1:200 and 1:300 for 3 different screening methods: screening by maternal age and first trimester NT (AN), by maternal age and first trimester maternal serum parameters (AMS) and by maternal age, NT and serum (ANMS). In the group of unaffected pregnancies, we defined for each screening method the false positive rate (FPR) at cut off 1:200 and 1:300 and the cut off value at $5 \%$ false positive screening results.

We searched the electronic database of Medline and PubMed for publications on population screening for fetal aneuploidy by first trimester combined algorithms, using the keywords Down's Syndrome, fetal aneuploidy, population screening, first trimester screening, combined screening, nuchal translucency, ultrasound screening, PAPP-A, HCG, maternal serum screening. According to the methodology reported in the materials and methods section, we grouped 
these studies in single ( $\leq 2$ centres) studies using FMF criteria for NT measurement, multicentre ( $\geq 3$ centres) studies using FMF criteria for NT measurement and studies using population-, group- or performer- specific NT medians.

\subsection{Results.}

The number of maternal serum screening tests in the AML-database increased from 9424 in 2000 to 12377 in 2003 . The 3-years evolution of the numbers and proportions of different tests is shown in Table 1.

Table 8.1. Evolution between 2000 and 2003 of the different screening tests pertormed by AML in Antwerp. Belgium (2AMS = maternal age + second trimester maternal serum parameters, $2 \mathrm{ANMS}=$ maternal age + muchal translucency + second trimester matemal serum parameters, 1 ANMS = maternal age + nuchal translucency + first trimester maternal serum parameters)

\begin{tabular}{|c|c|c|c|c|c|c|c|}
\hline & \multirow{2}{*}{$\frac{\text { Total }}{\mathbf{n}}$} & \multicolumn{2}{|c|}{ 2AMS } & \multicolumn{2}{|c|}{ 2ANMS } & \multicolumn{2}{|c|}{ 1ANMS } \\
\hline & & n & $(\%)$ & $\mathbf{n}$ & $(\%)$ & $\mathbf{n}$ & $(\%)$ \\
\hline 2000 & 9424 & 7502 & $(79.6)$ & 1797 & $(19.1)$ & 125 & (1.3) \\
\hline 2001 & 10534 & 6895 & $(65.4)$ & 1999 & $(19.0)$ & 1640 & (15.6) \\
\hline 2002 & 12107 & 7148 & $(59.0)$ & 1873 & $(15.5)$ & 3086 & $(25.5)$ \\
\hline 2003 & 12377 & 4053 & $(32.7)$ & 1747 & $(14.2)$ & 6577 & $(53.1)$ \\
\hline
\end{tabular}

All samples were obtained from a total of 264 obstetricians, of which only 6 were enlisted as FMF-accredited ultrasonographers at the FMF-website. The FMF-trainees measured $11.4 \%(1514 / 13267)$ of all NT values and all of them screened a minimum of 100 pregnancies. Of the other ultrasonographers, 31 screened $\geq 100$ pregnancies, 19 screened between 50 and 100 pregnancies and 209 screened less than 50 pregnancies. Blood was sampled $\geq 5$ days before the NT scan in $36.3 \%(4816 / 13267)$ of pregnancies. 
We evaluated a total of 13267 first trimester screening tests. In this total population, $26 \mathrm{~T} 21$ affected pregnancies and 23 pregnancies with other fetal chromosomal anomalies were present. Of the other pregnancies, a total of 49 were reported as intra-uterine fetal deaths, 12 before the 14th week of gestation, 23 between 14 and 24 weeks and 14 after 24 weeks. In these pregnancies, 2 fetal chromosomal anomalies different from T21 and 11 normal fetal karyotypes were reported. Five cytogenetic cell cultures failed. Four of the 12 first trimester miscarriages did not have any result on NT measurement, as the fetus died between the moment of blood sampling and the first trimester ultrasound. After exclusion of the 23 fetal chromosomal anomalies different from T21 and the 37 intra-uterine fetal deaths with unknown genetic constitution, a total of 13181 unaffected and $26 \mathrm{~T} 21$ - affected pregnancies were left for further evaluation.

Figure 8.1. "The correlation and correlation coefficient between the logarithmic values of our algonithm's risk calculations and the observed prevalence in our population.

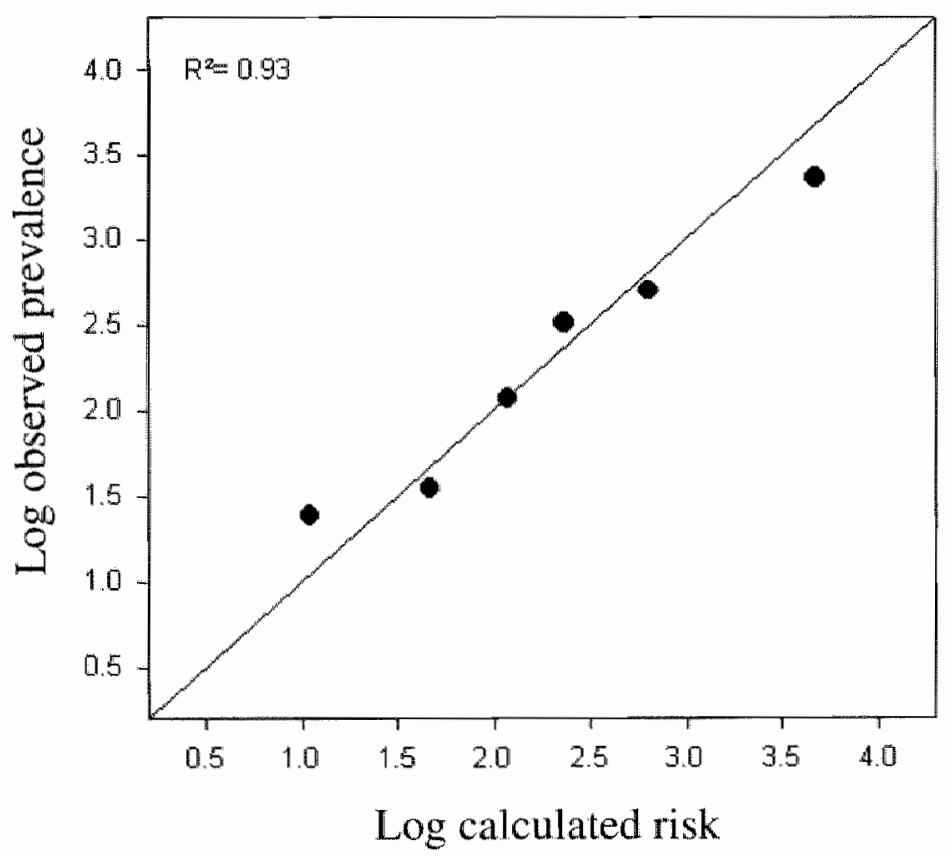


As is shown in Figure 1, the correlation between the logarithmic values of our algorithm's risk calculations and the observed prevalence of $T 2 l$ in our population was 0,93 .

We calculated the expected number of T21 at term from the maternal age distribution in our population and the maternal age related prevalence of T21 as registered in the British Down's Syndrome Cytogenetic Register (Morris et al., 2002b): the expected number of T21 at birth was 19,4. Considering a $43 \%$ spontaneous abortion rate, as reported by Morris et al. (Morris et al., 1999; Morris et al., 2002a) and assuming no intervention was performed for the 17 terminated T21-affected pregnancies in our population (Table 2), we calculated from our observations a total of 18.7 expected cases of T21 at term ( 9 live births +17 terminations $x$ 0.57). The correlation was $0,96(18,7 / 19,4)$ between the expected number of $\mathrm{T} 2 \mathrm{I}$ from our observations and the expected number from the maternal age distribution in our population.

In Table 2, details are enlisted on the different parameters and the screening results in the $26 \mathrm{~T} 21$-affected pregnancies. Of these pregnancies, a total of 17 were terminated and 5 T21-live births were reported. Four pregnancies with positive screening results were not terminated: after counselling, 2 women refused further invasive testing by amniocentesis or chorionic villus sampling and 2 other women accepted the birth of an affected child. Of all antenatal detected T21-affected pregnancies, $23.8 \%(5 / 21)$ were in the population screened by the FMF-trainees. The median MoM-values for all parameters were similar in the affected pregnancies screened by the FMF-trainees and the pregnancies screened by the other ultrasonographers.

From Table 2, the T21 detection rate (DR) at cut off 1:200 and 1:300 was defined for three different screening methods: screening by maternal age and NT (AN), by maternal age and maternal serum parameters (AMS) and by maternal age, NT and maternal serum (ANMS). 
Table 8.2. Details on the 26 T-21 affected pregnancies in our population: maternal age, gestation at blood sampling (Gestat $S$ ) in weeks and days (w,d), PAPP -A and free B- HCG (F BHCG) in mIU/mo and in corrected MoM-values, fetal Crown Rump Length (CRL) and nuchal translucency (NT) in mm and subseguent NT-MoM values, and the calculated risk by maternal age + NT (AN), by maternal age and serum (AMS) and by maternal age, NT and maternal serum (ANMS). $(T O P=$ termination of pregnancy, $L B=$ live birth). The 5 pregnancies screened by the FMF-accredited ultrasonographers are in bold.

\begin{tabular}{|c|c|c|c|c|c|c|c|c|c|c|c|c|}
\hline $\begin{array}{l}\text { Age } \\
\text { years }\end{array}$ & Gest: & $\mathrm{mIU} / \mathrm{ml}$ & $\begin{array}{c}\text { PAPP-A } \\
\text { Corr } \\
\text { MoM }\end{array}$ & $\begin{array}{c}\text { F B-HCG } \\
\text { mIU/ml }\end{array}$ & $\begin{array}{c}\text { F B-HCG } \\
\text { corr } \\
\text { MoM }\end{array}$ & CRL & NT & MoM & Risk & Risk & $\begin{array}{l}\text { Risk } \\
\text { ANMS }\end{array}$ & Out- \\
\hline 37,07 & 10,5 & 1,53 & 0,672 & 124,3 & 3,159 & 38,5 & 1,2 & 1,434 & 182 & 24 & 29 & TOP \\
\hline 39,05 & 10,3 & 0,38 & 0,261 & 14,6 & 0,311 & 37.4 & 0,5 & 0,627 & 423 & 23 & 129 & TOP \\
\hline 28,02 & 11,6 & 0,55 & 0,202 & 91,9 & 2,478 & 50,0 & 1,2 & 0,967 & 2921 & 28 & 67 & TOP \\
\hline 29,06 & 12.4 & 0,67 & 0,214 & 33,5 & 0,985 & 63,0 & 2,0 & 1,274 & 1292 & 80 & 127 & TOP \\
\hline 30,08 & 13.6 & 5,60 & 0,950 & 40,9 & 1,402 & 75,7 & 1,1 & 0,635 & 2820 & 1143 & 8386 & LB \\
\hline 36,03 & 12,5 & 3,05 & 0,610 & 266,8 & 7,059 & 63,2 & 1,9 & 1,207 & 457 & 12 & 23 & TOP \\
\hline 30,01 & 10,1 & 0,10 & 0,081 & 34,3 & 0,692 & 72,0 & 2,1 & 1,237 & 1317 & 11 & 15 & LB \\
\hline 30,02 & 13,2 & 0,95 & $0_{4} 257$ & 34,2 & 1,180 & 72,0 & 1,7 & 1,001 & 2232 & 99 & 299 & LB \\
\hline 30,00 & $1,2,4$ & 0,53 & 0,174 & 12,4 & 0,336 & 56,0 & 2,2 & 1.555 & 586 & 55 & 39 & TOP \\
\hline 41,08 & 12,3 & 1,38 & 0,378 & 1 & 8 & 69,0 & 2,9 & 1,744 & 26 & 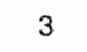 & 1 & 8 \\
\hline 38,00 & 11,3 & 0,85 & 0,418 & 96,2 & 2,221 & 51,0 & 1,7 & 1,336 & 213 & 22 & 32 & TOP \\
\hline 31,08 & 11,2 & 0,74 & 0,399 & 55,5 & 1,410 & 54,0 & 2,7 & 1,984 & 140 & 170 & 41 & TOP \\
\hline 31,07 & 11,2 & 3,82 & 1,606 & $34_{1}$ & $\int_{n}<09$ & 46,0 & 1,7 & 1,537 & 485 & 3219 & 4155 & $\mathrm{~B}$ \\
\hline 40,06 & 11,0 & 2,62 & 1,126 & 67,2 & 1,372 & 56,0 & 3,5 & 2,473 & 5 & 189 & 13 & LB \\
\hline 27,02 & 11,1 & 0,59 & 0,369 & $+0,<$ & 47 & 41,6 & 0,7 & 0,738 & 4016 & 404 & 2287 & W \\
\hline 33,00 & 12,0 & 0,62 & 0,224 & 69,4 & 1,759 & 52,5 & 1,0 & 0,759 & 1914 & 28 & 96 & TOP \\
\hline 22,03 & 10,6 & 1,07 & 0.730 & 220,9 & 4,405 & 40,0 & 4,0 & 4,490 & 1 & 82 & 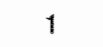 & TOP \\
\hline 26,10 & 12,0 & 1,54 & 0,710 & 17,8 & 0,484 & 60,6 & 1,0 & 0,657 & 4132 & 3028 & 28436 & LB \\
\hline 24,02 & 9,0 & 0,13 & 0,144 & 30,3 & 0,428 & 47,0 & 0,8 & 0,701 & $47+8$ & 48 & 200 & TOP \\
\hline 37,06 & 10,5 & 0,57 & 0,301 & 11.5 & 0,230 & 36,2 & 3,7 & 4,903 & 1 & 57 & 1 & TOP \\
\hline 28,06 & $1,2,3$ & 1,87 & 0,505 & 84,0 & 2,118 & 52,0 & 1,0 & 0,768 & 3598 & 196 & 865 & LB \\
\hline 32,08 & 12,4 & 1, & 94 & 8 & 49 & 65,0 & 2,9 & 1,807 & 190 & 86 & 00 & . \\
\hline 35,00 & 8,5 & 0,09 & 0,124 & 39,2 & 0,473 & 61,0 & 3,5 & 2,286 & 31 & 8 & i & TOP \\
\hline 32,08 & 11.1 & 0,71 & 0,320 & 170,2 & 3,290 & 48,0 & 2,3 & 1,958 & 125 & 21 & 6 & TOP \\
\hline 39,03 & 10,3 & 0,15 & 0,107 & 28,1 & 0,499 & 78,6 & 1,8 & 1,026 & 323 & 2 & 5 & TOP \\
\hline 27,04 & 11,6 & 1,80 & 0,659 & 4,6 & 0,820 & 48,9 & 4,4 & 3,652 & 3 & 1945 & 4 & 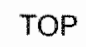 \\
\hline
\end{tabular}

\section{Medlian MoM's}

\begin{tabular}{llll} 
All & 0.37 & 1.29 & 1.31 \\
FMF trainees & 0.26 & 1.18 & 1.24 \\
Non FMF trainees & 0.38 & 1.40 & 1.34 \\
\hline
\end{tabular}


The results are shown in Table 3, which also shows the numbers of positive screening results and the False Positive Rate (FPR) in the unaffected pregnancies. The DR at a fixed 5\% FPR was $42.3 \%(11 / 26)$ for AN, $61.5 \%$ (16/26) for AMS and $73.6 \%$ (19/26) for ANMS at cut off values of respectively $1: 310,1: 85$ and $1: 180$.

Table 8.3. Performance of three different first trimester fetal aneuploidy screening methods: screening by maternal age + nuchal translucency (AN), by maternal age + serum (AMS) and by matemal age, nuchal translucency and serum (ANMS). The detection rate of trisony 21 (DR T21) and the false positive rate (FPR) at cut off 1:200 and 1:300 are enlisted, as well as the detection rate of T21 at a fixed $5 \%$ false positive rate and the cut off values at $5 \%$ FPR.

\section{$\underline{\text { DR T21 }} \quad \underline{\text { FPR }} \quad \underline{\text { DR T21 at 5\% FPR }}$}

\begin{tabular}{lllllll}
\multicolumn{4}{c}{$(\mathrm{n}=26)$} & \multicolumn{2}{c}{$(\mathrm{n}=13181)$} & $(\mathrm{n}=26)$ \\
\hline & $\mathbf{1 : 2 0 0}$ & $\mathbf{1 : 3 0 0}$ & $\mathbf{1 : 2 0 0}$ & $\mathbf{1 : 3 0 0}$ & cut off DR T21 \\
& $\mathbf{n}(\%)$ & $\mathbf{n}(\%)$ & $\mathbf{n}(\%)$ & $\mathbf{n}(\%)$ & $\mathbf{n}(\%)$ \\
AN & $10(38.5)$ & $11(42.3)$ & $419(3.2)$ & $635(4.8)$ & $1: 31.0$ & $11(42.3)$ \\
AMS & $21(80.8)$ & $21(80.8)$ & $1529(11.6)$ & $2212(16.8)$ & $1: 85$ & $16(61.5)$ \\
ANMS 20(76.9) & $21(80.8)$ & $724(5.5)$ & $1130(8.6)$ & $1: 180$ & $19(73.6)$ \\
\hline
\end{tabular}

\subsection{Discussion.}

In this report, we describe the introduction and the performance of our first trimester fetal aneuploidy screening program in Flanders (North-Belgium) and we compare our results with other publications.

As is shown in Table 1, the introduction of first trimester combined screening in our population was very efficient: within 3 years from introduction, first trimester combined screening became the most important serum-related screening method (Table 1). 
We validated our algorithm for the method of risk calculations and the method of registering the $T 21$-affected pregnancies. A good correlation $\left(R^{2}=0.93\right)$ was found between the logarithmic values of the algorithm's calculated risks and the observed prevalence of T21 in our population (Fig 1). We also found a good correlation $\left(R^{2}=0.96\right)$ between the expected number of $T 21$ - affected pregnancies from our observations and the expected number from the maternal age distribution in our population. We are aware that our analysis does not include the T21-affected pregnancies, missed by screening in the first trimester and resulting in a spontaneous fetal loss before term. It is likely that some chromosomal defects were present in our group of 37 pregnancies with fetal loss of unknown genetic constitution. Therefore, our calculations on the algorithm's performance may be overestimating the detection of T21. This problem of inaccuracy in reporting screening performance of first trimester screening methods has already been highlighted before (Haddow, 1998). Our approach however is similar to the methods used in most reports enlisted in Table 4 , which allows for comparison of our data with the results from these studies.

As shown in Table 3, the performance of screening by AN shows a 42.3\% T21 detection rate at 5\% FPR. This is considerably less than the $75-80 \%$ DR deducted from a series of publications on NT screening in unselected populations (Brigatli and Malone, 2004). In our algorithm, we did not use population-, centre- or performer- specific reference values for NTmeasurements, as proposed by others (Logghe et al, 2003; Wald et al, 2003). We implemented NT-values, expressed as a Multiple of the Median (MoM) relative to the FMF- reference range. We recently reported a systematic underestimation of the NT-MoM values in our database, in comparison to this FMF- reference range (Gyselaers et al, 2004a). This underestimation may explain the poor performance of AN screening in our population. FMF-trained ultrasonographers performed less than $12 \%$ of all NT measurements in our population. Of the other ultrasonographers, no information was available on 
their ultrasound qualifications and the majority of them screened less than 50 pregnancies in the 3-years study period. The implementation of NT measurements, without active training and quality control, was reported to be unreliable in combination with first trimester maternal serum screening (Haddow et al., 1998). In a careful review of NT screening studies by Malone et al. (Malone and D'Alton, 2003), there was a broad range of detection rates and false positive rates in 30 studies of unselected patient populations. The performance of the combined algorithm ANMS in our study showed an acceptable T21 DR of $80.8 \%$ at FPR of $8.6 \%$, which was similar to other multicentre studies (Muller et al., 2003; Niemimaa, 2003; Wald et al., 2003; Wapner et al., 2003). As is shown in Table 3, in our algorithm the T2』 DR was mainly achieved by AMS only. NT did not add to this DR, but was an important parameter to reduce FPR from $11.6 \%$ tot $5.5 \%$ at cut off $1: 200$ and from $16.8 \%$ to $8.6 \%$ at cut off 1:300 (Table 3 ). The median MoM values of PAPP-A in our group of affected pregnancies were appropriately low (Table 2). The median MoM values of FB-HCG and NT were not as elevated as expected: it is likely that higher values of one or both would improve our screening results. NT MoM medians were not different between the pregnancies screened by the FMF trainees and those screened by the other ultrasonographers: we think this is coincidental. From these data, it is concluded that PAPP-A is by far the most important screening parameter in our algorithm. Muller et al. reported different screening results from second trimester maternal serum screening algarithms, depending on the relative weight of the parameters (Muller et al., 1999). In our algorithm, the relative weight of NT is low, and this may explain the minor impact of underestimated NT values on the final screening results of our combined algorithm. A theoretical calculation in the SURUSS report also showed a minor impact of increasing fractions of sub optimal NTmeasurements on the final results of combined or integrated population screening (Wald et al., 2003). The results from our study add to the growing evidence that NT measurements, performed under different conditions as 
recommended by FMF, may still be valuable in fetal aneuploidy screening, when used in combination with other parameters.

Table 8.4. List of publications of prospective population studies on first trimester combined fetal aneuploidy screening (Bindra et al, 2002;Borrell et al., 2004; Crossley et al, 2002; De Biasio et al., 1999; Krantz et al., 2000; Muller et al., 2003; Niemimaa, 2003; Orlandi et al., 1997; Schuchter et al, 2002; Scott et al, 2004;Spencer et al., 2003;Stenhouse et al., 2004; won Kaisenberg et al., 2002; Wald et al, 2003; Wapner et al., 2003). The studies are grouped in (1) single centre ( $\leq 2$ centres) studies with FMF scanning criteria, (2) multicentre ( $\geq 3$ centres) studies with FMF scanning criteria and (3) studies with population specific NT-Medians.

\begin{tabular}{|c|c|c|c|c|c|c|c|c|}
\hline \multirow[t]{2}{*}{ Author } & \multirow[t]{2}{*}{ Centres } & \multirow[t]{2}{*}{$\mathbf{n}$} & \multirow[t]{2}{*}{ cut off } & \multicolumn{2}{|c|}{ Detection rate } & \multicolumn{2}{|c|}{ False Pos rate } & \multirow{2}{*}{$\begin{array}{c}\text { DR at } 5 \% \\
\text { FPR } \\
\%\end{array}$} \\
\hline & & & & $\%$ & $(n / n)$ & $\%$ & $(\mathrm{n} / \mathrm{n})$ & \\
\hline
\end{tabular}

Single centre - FMF

$\begin{array}{cccccccc}\text { Stenhouse } & 5084 & 1 / 250 & 93 & 14 / 15 & 5,5 & 274 / 4974 & \\ \text { Scott } & 2121 & 1 / 300 & 100 & 5 / 5 & 7,1 & 148 / 2104 & . \\ \text { Spencer } & 12339 & 1 / 300 & 92 & 23 / 25 & 5,2 & 577 / 11105 & 89 \\ \text { Krantz } & 5809 & 1 / 270 & 91 & 30 / 33 & 8,6 & 451 / 5223 & 91 \\ \text { Bindra } & 14383 & 1 / 300 & 91 & 75 / 82 & 6,8 & 967 / 14240 & 90 \\ \text { Schuchter } & 4939 & 1 / 250 & 86 & 12 / 14 & 5 & 245 / 4925 & 86 \\ \text { Orlandi } & 2010 & 1 / 380 & 100 & 11 / 11 & 9 & 165 / 1833 & 87 \\ \text { DeBiasio } & 1467 & 1 / 350 & 85 & 11 / 13 & 3,3 & 48 / 1454 & . \\ & 48152 & & 91,4 & 181 / 198 & 6,3 & 2875 / 45858 & 89,5\end{array}$

Multicentre - FMF

\begin{tabular}{ccccccccc} 
Wapner & 12 & 8216 & $1 / 270$ & 85,2 & $52 / 61$ & 9,4 & $766 / 8144$ & 79 \\
V Kaisenb & 9 & 3864 & $1 / 300$ & 84 & $16 / 19$ & 6,6 & $233 / 3505$ & $74-84$ \\
Crossley & 25 & 17229 & $1 / 250$ & $62-82$ & $28 / 34-45$ & 5 & $(649 / 12983)^{*}$ & 82 \\
& $\mathbf{4 6}$ & $\mathbf{2 9 3 0 9}$ & & $\mathbf{8 2 , 5}$ & $\mathbf{9 4 / 1 1 4}$ & $\mathbf{6 , 7}$ & $\mathbf{1 6 4 8 / 2 4 6 3 2}$ & $\mathbf{7 9 , 9}$ \\
\hline
\end{tabular}

Population specific reference

\begin{tabular}{ccccccccc} 
Borrell & 1 & 2780 & $1 / 250$ & 88 & $7 / 8$ & 3,3 & $92 / 2765$ & 88 \\
Niemimaa & 2 & 3178 & $1 / 250$ & 77 & $10 / 13$ & 5,8 & $184 / 3165$ & 77 \\
Muller & 9 & 5694 & $1 / 250$ & 74 & $19 / 26$ & 4,7 & $265 / 5644$ & 77 \\
SURUSS & 25 & 39983 & $1 / 250$ & 80 & $65 / 85$ & 4,7 & $1875 / 39898$ & 83 \\
& $\mathbf{3 7}$ & $\mathbf{5 1 6 3 5}$ & & $\mathbf{7 6 , 5}$ & $\mathbf{1 0 1 / 1 3 2}$ & $\mathbf{4 , 7}$ & $\mathbf{2 4 1 6 / 5 1 4 7 2}$ & $\mathbf{8 1 . 5}$ \\
\hline
\end{tabular}

* Numbers deducted from the original data 
Table 4 enlists publications of population studies on combined first trimester screening by matemal age, NT, PAPP-A and free B-HCG, grouped by the number of centres involved in each study and the methodology used for implementation of NT measurements. The overall prevalence of $T 21$ in the single centre studies ( $\leq 2$ centres) is $1 / 243$ (198/48152), in the multicentre studies ( $>2$ centres) using FMF criteria for NT measurement is 1/257 (114/29309) and in the studies using population-specific NT medians is $1 / 411$ (158/64904). As is shown, the overall performance of the algorithms using population specific medians is lower than the overall performance of the algorithms from the single centre studies using FMF criteria. However, when the FMF criteria are used in multicentre studies, the overall screening performance is also lower than in the single centre studies and equal to the overall performance of algorithms with population specific NT medians: for both methods the T21 DR at 5\% FPR varies around $80 \%$ compared to $89.5 \%$ for the single centre studies (Table 4). The 3 multicentre studies with FMF criteria reported difficulties with compliance to the protocol for ultrasonic NT measurement. This clearly illustrates the difficulty to introduce stringent US methods in population screening for fetal aneuploidy and to move the expertise from the specialist centres to antenatal screening clinics. However, the Scottish group recently reported that an optimal performance of first trimester combined screening was achievable in a routine antenatal clinic after motivation for adherence to an ultrasound screening protocol and reorganisation of busy antenatal clinic activities (Stenhouse et al., 2004).

At present, the bottle neck in the organisation of first trimester population screening for fetal aneuploidy is the availability of appropriately trained and equipped ultrasonographers (Benn, 2002). Both in the UK as in the USA, it was reported that only a minority of pregnant women had access to high quality NTrelated screening (Brigatti and Malone, 2004;Egan et al., 2002; Welch and Malone, 2003; Whittle, 2001). In our population, only $11.4 \%$ of the pregnant women had access to NT measurements according to FMF criteria and this 
allowed a detection of less than $20 \%$ of all $\mathrm{T} 21$-affected pregnancies in the population. Programs have been developed towards training and audit of an increasing number of obstetric ultrasonographers, in order to offer high quality ultrasound examinations to as many pregnant women as possible (Braithwaite et al., 1996; Snijders et al., 2002; Wojdemann et al., 2001). The medical and ethical implications of the general introduction of these Programs for Certification of Competence have been highlighted (Wald, 2003). Our data show that unspecified NT measurements for fetal aneuploidy screening are easier to introduce in population screening than those according to FMF criteria. Our data also show that this practice does not necessarily implly poor medical practice, as the screening results from our algorithm are acceptable and comparable to other population screening studies, using FMF-criteria (Wapner et al., 2003) or population-specific medians (Muller et al., 2003;Niemimaa, 2003; Wald et al., 2003). Finally, our data show that the large number of women screened by the non-FMF-trained ultrasonographers allowed to detect 3 times the number of $\mathrm{T} 21$ affected pregnancies detected by the FMF-trainees.

We acknowledge however the importance of further improving the screening performance of our screening program. We are currently investigating four measures: (1) an increase of the number of parameters, such as the ultrasonic evaluation of the fetal nasal bone (Cicero et al., 2003) and/or the integration of first and second trimester parameters (Wald et al., 2003), (2) the application of population-, centre- or performer-specific reference values and correlations for all screening parameters (Logghe et al., 2003; Wald et al., 2003), (3) further training and audit on ultrasound methodology for obstetricians involved in first trimester ultrasound screening (Braithwaite et al., 1996;Snijders et al, 2002), which has already started following a survey on the future organisation of ultrasound screening in Flanders (Gyselaers et al., 2005) and (4) the introduction of contingent testing, which offers maternal serum screening to all pregnant women in the program, and subsequent high quality ultrasound 
screening to only those who are considered at higher nisk (Christiansen and Olesen, 2002).

We conclude that the introduction of first trimester combined screening with unspecified ultrasound methodology in a Belgian population was very easy. The performance of this screening method was less than in reported single centre studies using FMF scanning criteria, but the easy access to screening and the contribution from maternal serum parameters were responsible for the majority of T21 detections in our population. Our data illustrate that easy access to screening should be balanced against the uniform application of a single ultrasound protocol, in order to identify the highest possible number of fetal aneuploidy affected pregnancies in the population.

\section{References}

Benn, P. A. (2002) Advances in prenatal screening for Down syndrome: II first trimester testing, integrated testing, and future directions. Clin Chim Acta 324: 1-11.

Bindra, R., Heath, V., Liao, A., Spencer, K., and Nicolaides, K. H. (2002) One-stop clinic for assessment of risk for trisomy 21 at $11-14$ weeks: a prospective study of 15030 pregnancies. Utrasound Obstet Gynecol 20:219-225.

Borrell, A., Casals, E., Fortuny, A., Farre, M. T., Gonce, A., Sanchez, A., Soler, A., Cararach, V., and Vanrell, J. A. (2004) First trimester screening for trisomy 21 combining biochemistry and ultrasound at individually optimal gestational ages. An interventional study. Prenat Diagn 24: $541-545$.

Braithwaite, J. M., Kadir, R. A., Pepera, T. A., Morris, R. W., Thompson, P. J., and Economides, D. L. (1996) Nuchal translucency measurement: training of potential examiners. Uitrasound Obstet Gynecol 8: 192-195.

Brigatti, K. W. and Malone, F. D. (2004) First-trimester screening for aneuploidy. Obstet Gynecol Clin North Am 31: 1-20.

Christiansen, M. and Olesen, Larsen S. (2002) An increase in cost-effectiveness of first trimester matemal screening programmes for fetal chromosome anomalies is obtained by contingent testing. Prenat Diagn 22:482-486.

Cicero, S., Bindra, R., Rembouskos, G., Spencer, K., and Nicolaides, K. H. (2003) Integrated ultrasound and biochemical screening for trisomy 21 using fetal nuchal translucency, absent fetal nasal bone, free beta-hCG and PAPP-A at 11 to 14 weeks. Prenat Diagn 23: 306-310. 
Crosslky, J. A., Aitken, D. A., Cameron, A. D., McBride, E., and Connor, J. M. (2002) Combined ultrasound and biochemical screening for Down's syndrome in the first trimester: a Scottish multicentre study. BJOG $\| 09: 667-676$.

De Biasio, P. Siccardi, M., Volpe, G., Famularo, L., Santi, F., and Canini, S. (1999) Firsttrimester sereening for Down syndrome using nuchal translucency measurement with free bellahCG and PAPP-A between 10 and 13 weeks of pregnancy-the combined test. Prenat Diagn 19: 360-363.

de Graaf, I. M.., Cuckle, H. S., Pajkrt, E., Leschot, N. J., Bleker, O. P., and van Lith, J. M. (2000) Comvariables in first trimester maternal serum screening. Prenat Diagn 20: 186-189.

de Graaf, I. M., Pajkrt, E., Bilardo, C. M., Leschot, N. J., Cuckle, H. S., and van Lith, J. M. (1999) Early pregnancy screening for fetal aneuploidy with serum markers and nuchal translucency. Prenar Diagn 19: 458-462.

Egan, J. F., Kaminsky, L. M., DeRoche, M. E., Barsoom, M. J., Borgida, A. F., and Benn, P. A. (2002) Antenatad Down syndrome screening in the United States in 2001: a survey of maternalfetal medicine specialists. Am J Obstet Gynecol 187: 1230-1234.

Gyselaers, W., De Catte, L., Witters, I., De Jonge, E., Ombelet, W., and Nijhuis, J. (2005) Survey on the future organisation of first trimester ultrasound screening in Flanders, Belgium. Prenat Diagn 25: $518-59$.

Gyselaers, W., Vereecken, A., Van Herck, E., Straetmans, D., De Jonge, E., Ombelet, W., and Nijhuis, J. (2004a) Audit on nuchal translucency thickness measurements in Flanders, Belgium: a plea for methodological standardization. Uhrasound Obstet Gynecol 24:511-515.

Gyselaers, W., Vereecken, A., Van Herck, E., Straetmans, D., Martens, G., De Jonge, E., Ombelet, $W .$, and Nijhuis, J. (2004b) Screening for trisomy 21 in Flanders: a 10-years review of 40490 pregnancies screened by maternal serum. Eur J Obstet Gynecol Reprod Biol 115: 185-189.

Haddow, J. E. (I-8-1998) Antenatal screening for Down's syndrome: where are we and where mext? Lancet 352: 336-337.

Haddow, J. E., Palomaki, G. E., Knight, G. J., Williams, J., Miller, W. A., and Johnson, A. (2.4. 1998 ) Screening of matenal serum for fetal Down's syndrome in the first trimester. $N$ Engl J Med 338: $955-961$.

Hallahan, T., Krontz, D., Buchanan, P., Larsen, J. W., and Macri, J. Effect of ethnicity of firsttrimester Down Syndrome screening. htp://genetics.fuseb.org/genetics/ashg00/2365.htm * (accessed 20-5/2004).

Krantz, D. A., Hallahan, T. W., Orlandi, F., Buchuman, P., Larsen, J. W., Jr., and Macri, J. N. (2000) First-trimester Down syndrome screening using dried blood biochemistry and nuchal translucency. Obster Gynecol 96: 207-213.

Logghe, H., Cuckle, H., and Sehmi, I. (2003) Centre-specific ultrasound nuchal translucency medians needed for Down syndrome screening. Prenat Diagn 23: 389-392.

Malone, F. D. and D'Alton, M. E. (2003) First-trimester sonographic screening for Down syndrome. Obster Gynecol 102: 1066-1079. 
Morris, J. K., Mutton, D., and Alberman, E. (2002a) Potenthal biases in Down syndrome birh prevalence estimation (author's reply). I Med Screen 9: 192.

Morris, J. K., Mutton, D. E., and Alberman, E. (2002b) Revised estimates of the maternal age specific live birth prevalence of Down's syndrome. J Med Screen $9: 2-6$.

Morns, J. K., Wald, N. J., and Watt, H. C. (1999) Fetal loss in Down syndrome pregnancies. Prewat Diagn 19: 142-145.

Muller, F., Aegerter, P., Ngo, S., Fort, A., Beauchet, A.x Giraudet, P., and Dommergues, M. (1999) Software for prenatal down syndrome risk calculation: a comparative study ol six software packages. Clin Chem 45: 1278-1280

Muller, F., Benattar, C., Audibert, F. Roussel, N., Dreux, S., and Cuckle, H. (2003) Firsttrimester screening for Down syndrome in France combining fetal nuchal transhucency measurement and biochemical markers. Prenat Diagn $23: 833-836$.

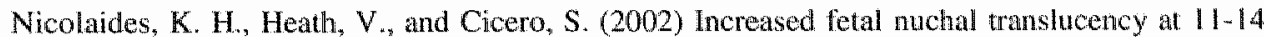
weeks. Prenat Diagn 22: 308-315.

Niemimat, M. First trimester screening for Down Syndrome 1-62.27-6-2003. Thesis, University of Oulu, Finland.

Onda, T., Tanaka, T., Takeda, O., Kitagawa, M., Kuwabara, Y., Yamamoto, H., linuma, K., and Shimomura, K. (1998) Agreement between predicted risk and prevalence of Down syndrome in second-trimester triple-marker screening in Japan. Prenat. Diagn 18: 956-958.

Orlandl, F., Damiani, G., Hallahan, T. W., Krantz, D. A., and Macri, J. N. (1997) First-trimester screening for fetal aneuploidy: biochemistry and nuchal translucency. Uitrasound Obstet Gynecol 10: $381-386$.

Schuchter, K., Hafner, E., Stangl, G., Metzenbauer, M., Hofinger, D., and Philipp, K. (2002) The first trimester "combined test" for the detection of Down syndrome pregnancies in 4939 unselected pregnancies. Prenat Diagn 22: $21111-215$.

Scott, F., Peters "H., Bonifacio, M., Mclemman, A., Boogert, A., Kesby, G., and Anderson, J. (2004) Prospective evaluation of a first trimester screening program for Down syndrome and other chromosomal abnormalities using maternal age, nuchal translucency and biochemistry in an Australian population. Atst N Z J Obstet Gynaecol 44: 205-209.

Snijders, R. J., Thom, E. A., Zachary, J. M., Platt, L. D., Greene, N., Jackson, L. G., Sabbagha, R. $\mathbb{E}$, Filkins, K., Silver, R. K., Hogge, W. A., Ginsberg, N. A., Beverly, S., Morgan, P., Blum, K., Chilis, P., Hill, L. M., Hecker, J., and Wapner, R. J. (2002) First-irimester trisomy screening: nuchal translucency measurement training and quality assurance to correct and unify technique. Ultrasound Obstet Gynecol 19: 353-359.

Spencer, K. (2000) Screening for trisomy 21 in twin pregnancies in the first trimester using free beta-hCG and PAPP-A, combined with fetal nuchal tanslucency thickness. Prenat Diagn $20: 91$. 95.

Spencer, K., Spencer, C. E., Power, M., Dawson, C., and Nicolaides, K. H. (2003) Screening for chromosomal abnormalities in the first trimester using ultrasound and maternal serum 
biochemistry in ane-stop clinic: a review of three years prospective experience. BJOG 110:281286.

Stenhouse, E. J., Crossley, J. A. Aitken, D. A., Brogan, K., Cameron, A. D., and Connor, J. M. (2004) First-trimester combined ultrassound and biochemical screening for Down syndrome in routine clinical practice. Prenat Diagn 24: 774-780.

won Kaisenberg, C. S., Gasiorek-Wiens, A., Bielicki, M., Bahlmann, F., Meyberg, H., Kossakiewicz, A., Pruggmayer, M., Kamin, G., Fritzer, E., Harris, C., and Arnold, N. (2002) Screening for trisomy 21 by maternal age, fetal nuchal translucency and maternal serum biochemistry at 11-14 weeks: a German multicenter study. J Matern Fetal Neonatal Med 12:8994.

Wald, N. (2003) Certification of competence in performing specific procedures or tests in screening practice Prenat Diagn 23: 861 .

Wald, N. J., Kennard, A., Hackshaw, A., and McGuire, A. (1997) Antenatal screening for Down's syndrome. I Med Screen 4: 181-246.

Wald, N. J., Rodeck, C., Hackshaw, A. K., Walters, J., Chitty, L., and Mackinson, A. M. (2003) First and second trimester antenatal screening for Down's syndrome: the results of the Serum, Urine and Ultrasound Screening Study (SURUSS). J Med Screen 10: 56-104.

Wapner, R., Thom, E., Simpson, J. L., Pergament, E., Silver, R., Filkins, K., Platt, L., Mahoney, M., Johnson, A., Hogge, W. A., Wilson, R. D., Mohide, P., Hershey, D., Krantz, D., Zachary, J., Snijders, R., Greene, N., Sabbagha, R., MacGregor, S., Hill, L., Gagnon, A., Hallahan, T., and Jackson, L. (9-10-2003) First-trimester screening for trisomies 21 and 18 . N Engl J Med 349: $1405-1413$.

Welch, K. K. and Malone, F. D. (2003) Nuchal translucency-based screening. Clin Obstet Gynecol 46: 909-922.

Whittle, M. (2001) Down's syndrome screening: where to now? BJOG 108: 559-561.

Wojdemann, K. R., Christiansen, M., Sundberg, K., Larsen, S. O.., Shalmi, A., and Tabor, A. (2001) Quality assessment in prospective nuchal translucency screening for Down syndrome. Utrasound Obstet Gynecol 18: 641-644. 
Chapter 9

\title{
Nuchal translucency thickness
}

\section{measurements for fetal}

\section{aneuploidy screening:}

\author{
Log NT-MoM or Delta-NT,
}

performer-specific medians and ultrasound training

Gyselaers WJA, Vereecken AJ, Van Herck EJH, Straetmans DPL. Ombelet WUAM, Nijhuis JG.

(Submitted). 


\section{Summary}

Objectives. To evaluate in fetal aneuploidy screening the desirability of using Fetal Medicine Foundation (FMF) normal medians of nuchal translucency thickness measurements (NT) or performer-specific medians, and whether the NT- measurements should be expressed as Delta-NT or Log NT-MoM values. Settings. First trimester combined screening program in a low risk population in Flanders, Belgium (Algemeen Medisch Laboratorium (AML), Antwerp). Methods. Trisomy 21 (T21) -unaffected pregnancies were screened by FMFtrained or other ultrasonographers. Performer- specific NT medians were established for FMF-trained and other ultrasonographers. NCSS Statistical Software was used to establish probability plots for Log NT-MoM and DeltaNT values, relative to performer-specific references or to the FMF-reference. Results. A total of 16096 pregnancies was evaluated. Six FMF-trainees and 5 other ultrasonographers performed each between 83 and 658 NTmeasurements. For the FMF-trainees, FMF-specific NT- MoM medians were close to 1 at crown-rump length (CRL) between 50 and $80 \mathrm{~mm}$, whereas this was at CRL between 40 and $80 \mathrm{~mm}$ for the population-specific NT-MoM medians of the other ultrasonographers. Between the $50^{\text {th }}$ and $95^{\text {th }}$ percentile, the NT- probability plots were almost identical for the FMF-specific values of the FMF-trainees and the performer-specific values of the non-FMF-trainees. Conclusion. We conclude that the use of population- or performer-specific NTmedians for calculation of Delta-NT or Log NT-MoM values allows to implement NT-measurements in fetal aneuploidy screening algorithms at earlier gestation than 11 weeks. The probability plots for FMF-specific NTmeasurements of FMF-trainees and performer-specilic values of other ultrasonographers are nearly identical in the upper, most important range of the NT- measurements. 


\subsection{Introduction.}

In fetal aneuploidy screening today, mathematic algorithms are used to calculate an individual's risk for fetal chromosomal abnormalities, using parameters derived from maternal serum and/or obtained by ultrasound. As the measured values for every parameter vary throughout gestation, the observed values are expressed as a function of the median at the same gestation, either as a multiple $(\mathrm{MoM})^{\prime}$ or as a difference (Delta) ${ }^{2}$. For fetal nuchal translucency thickness (NT) measurements, the measured values are expressed in relation to the median values at the corresponding fetal Crown-Rump Lengths (CRL). The CRLrelated medians can be derived from a standard reference curve, such as the one published by the Fetal Medicine Foundation (FMF) centre- or population specific ${ }^{4 ; 5}$.

In the fetal aneuploidy screening program organised by the Algemeen Medisch Laboratorium AML in Antwerp, Belgium, NT related screening was introduced in $1999^{\circ}$. In this program, NT was implemented into first or second trimester screening algorithms, expressed as logarithmic NT-MoM values ( $\log _{10} \mathrm{NT}$ MoM) in relation to the FMF reference range. We reported the results of an audit on the methodology of NT measurement in this program, by comparing the observed Delta-NT values with those from the standard FMF curve ${ }^{7}$. This audit showed a systematic underestimation of the NT- measurements by the ultrasonographers who were not enlisted as FMF-trainees at the official FMFwebsite, as compared to the measurements of the ultrasonographers who were on this list.

In the present study, we compare the performer-specific CRL-related NTmedians from the FMF-trainees with those from the other ultrasonographers. We also evaluate the effect on the NT- distribution curves of introducing population- or performer- specific median NT-values for calculation of NTMoM or Delta-NT values. 


\subsection{Materials and methods.}

Between 1/1/2001 and 30/4/2004, maternal serum samples for first trimester fetal aneuploidy screening have been analysed by AML. These samples were recruited from 264 obstetricians, active in 35 centres located in all geographic regions of Flanders. All serum samples were accompanied with data on first trimester NT and CRL in $\mathrm{mm}$. The first trimester ultrasound scans were performed by the obstetricians, referring the blood samples for analysis. The FMF-accredited ultrasonographers were identified at the official FMF-website (http://www.fetalmedicine.com/f-competence.hum; accessed 1/1/2003 to $1 / 5 / 2005)$.

Our methods of data collection and risk calculation are reported elsewhere 7 According to outcome of pregnancy, all data were grouped in trisomy 21 (T21) affected pregnancies, pregnancies with fetal chromosomal anomalies different from T21, pregnancies with fetal loss of unknown genetic constitution and unaffected pregnancies. In this study, only unaffected pregnancies were considered.

A total of 2700 NT/Crown rump length (CRL) measurements were mailed to FMF. From these data, CRL-related Multiples of the Median (NT-MoM) were mailed back to us, which we used to establish the FMF reference curve of gestation related NT-values. We also received from FMF the NT-MoM-values at CRL measurement below $45 \mathrm{~mm}$, but a note was added to consider the FMFreference range only at CRL values between 45 and $85 \mathrm{~mm}$. We also established a population-specific reference curve of CRL-related median NT-values from all unaffected pregnancies, screened by 258 ultrasonographers who were not enlisted at the FMF website (AML reference). Finally, we established a performer-specific curve of CRL-related NT- medians for every individual FMF- trained ultrasonographer and for 5 non-FMF-trainees, who performed the most numerous screening tests in the AML screening program. 
Figure 9.1.A and B. Median values of Nuchal Translucency (NT) thickness measurements in relation to fetal Crown-Rump-Length (CRL), for a group of 6 ultrasonographers, trained by the Fetal Medicine Foundation (FMF) $(\mathrm{n}=1513)$ and for a group of 258 other ultrasonographers $(n=14583)$. In Figure $1 \mathrm{~A}$ (upper), NT multiples of the median (NT-MoM) are calculated in relation to the FMF-reference range. Figure 1B (lower) shows the same data, as calculated in relation to the AML population-specific reference range.
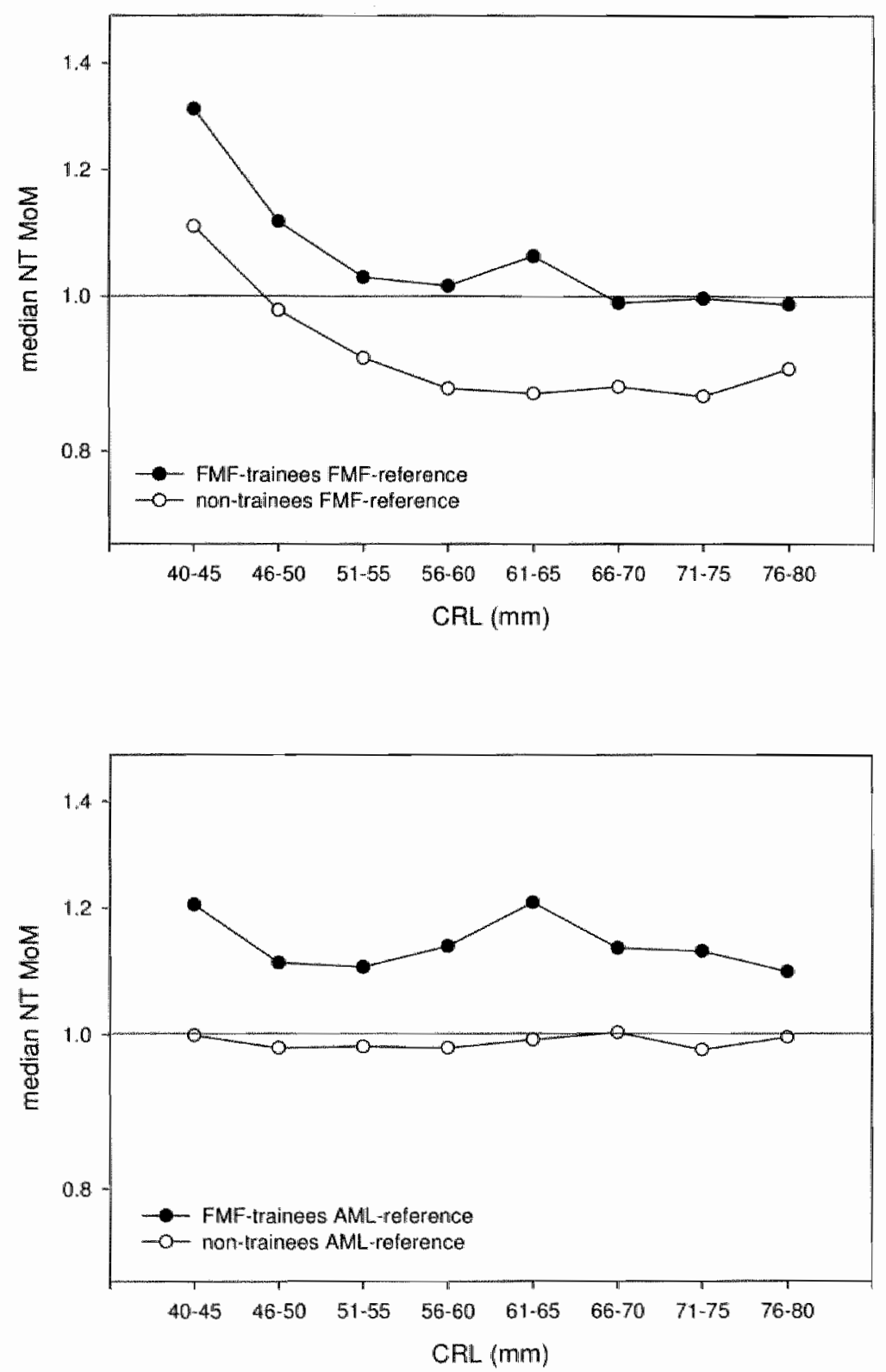
We used both the FMF-medians, the AML-medians and the performer-specific medians to calculate values of Log NT-MoM and Delta-NT. NCSS Statistical Software (329 North 1000 East-Kaysville, Utah, USA) was used to establish probability plots for all NT- measurements expressed as Log NT-MoM or DeltaNT, relative to the FMF-reference or to AML-specific or performer-specific reference values. All distribution curves were plotted graphically. The FMFspecific Delta-NT and Log NT-MoM probability plots of one FMF- trained ultrasonographer were compared with the Delta-NT and Log NT-MoM curves of the 5 non-FMF-trained ultrasonographers, whose NT- measurements were expressed in relation to performer-specific medians.

\subsection{Results.}

We evaluated NT-measurements from a total of 16096 unaffected pregnancies. These measurements were performed by a total of 264 ultrasonographers, of which 6 were enlisted at the FMF website between $1 / 1 / 2003$ and 1/5/2004. FMF-trainee A, B, C, D, E and F performed respectively 600, 352, 144, 140, 194 and 83 NT- measurements. The 5 most active non-FMF-trained ultrasonographers in the AML screening program performed respectively 483 (A), 658 (B), 518 (C), 262 (D) and 361 (E) NT- measurements. The NTmeasurements of all other ultrasonographers were not evaluated individually.

Figure 1 shows the plot of CRL-related NT- measurements of the group of FMF-trainees and of the group of all other ultrasonographers, expressed as NTMoM. The FMF-trainees have systematically higher NT-medians compared to the other ultrasonographers. Figure 1A shows the NT- measurements, expressed in relation to the FMF reference range, whereas Figure $1 B$ shows the same measurements, expressed in relation to the population-specific reference range. In Figure $1 \mathrm{~A}$, the median NT-MoM of the FMF-trainees is around 1 at CRL between 50 and $80 \mathrm{~mm}$, but is above 1 at lower CRL values. In Figure 1B, the median NT-MoM is around 1 at CRL between 40 and $80 \mathrm{~mm}$. 
Fig 9.2.A and B. Medians of Nuchat Translucency (NT) thickness measurements (in mm) in relation to fetal Crown-Rump-Length (CRL), for 5 ultrasonographers, not enlisted as trainee at the Fetal Medicine Foundation (FMF) website (Fig A, upper), and for 6 FMF-trainees (Fig B, lower).
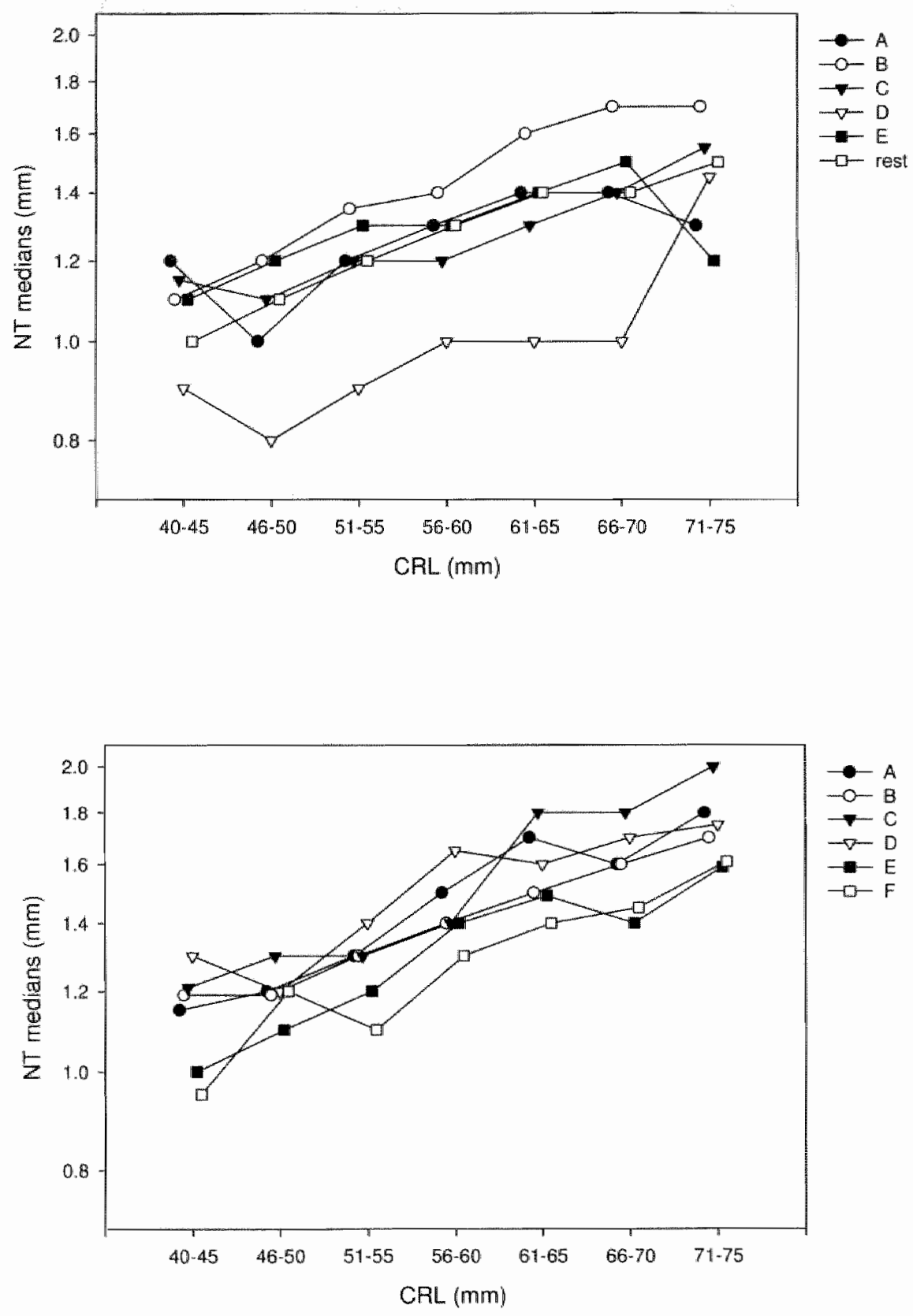
Figure 2 shows the CRL-related NT medians (in $\mathrm{mm}$ on a log scale) for every individual FMF- trainee (Figure 2B) and for 5 other ultrasonographers (Figure 2A). As is shown, all performer-specific curves are different, both in the group of FMF-trainees and in the group of other ultrasonographers. However, the interobserver variation is less in the group of FMF-trainees compared to the other ultrasonographers. The curves of 4 of the non-FMF-trainees are relatively close to those of the FMF-trainees, whereas non-trainee D (open triangles) has systematically very low NT-measurements.

The probability plots of NT-measurements are shown in Figure 3, either expressed as Delta-NT (Figure 3A) or Log NT-MoM (Figure 3B). In both graphs, 6 different curves are plotted: one from FMF-trainee A (see Figure 2B), whose measurements are expressed in relation to FMF-specific medians, and 5 from the non-FMF-trained ultrasonographers (see Figure 2A), whose measurements are expressed in relation to performer-specific medians. As is shown, all Delta-NT curves are nearly identical. The Log NT-MoM curves are slightly different, but this difference is mainly in the lower range of the distribution curves $\left(<40^{\text {th }}\right.$ centile). Between the $50^{\text {th }}$ and $95^{\text {th }}$ centile, the FMFspecific curves of the FMF-trainee and the performer-specific curves of the other ultrasonographers are nearly identical.

\subsection{Discussion.}

In the fetal aneuploidy screening program organised by AML, Antwerp Belgium, NT- measurements were introduced as a screening parameter in 1999, first in combination with second trimester triple serum parameters ${ }^{6}$ and later also with first trimester serum screening ${ }^{8}$. In these algorithms, NTmeasurements are expressed as Log NT-MoM, relative to a standard reference range, established by $\mathrm{FMF}^{3}$. Our audit on NT-measurements, as used in our program, showed a systematic underestimation of NT- measurements from a group of ultrasonographers, not enlisted as FMF-trainees at the FMF website, compared to the measurements of FMF- trainees ${ }^{7}$. 
Figure 9.3.A and B. Probability plot of NT-measurements from FMF-rainee A (see Figure 2B) and from 5 non-FMF-trained ultrasonographers (see thgure $2 \mathrm{~A}$ ), expressed as DeltaNT (Figure 3A) or Log NT-MoM (Figure 3B). For the FMF-trainee, Delta-NT or Log NT-MoM values are expressed in relation to the FMF-specific reference range, whereas for the non-FMFtraneess, performer-specific values are shown.
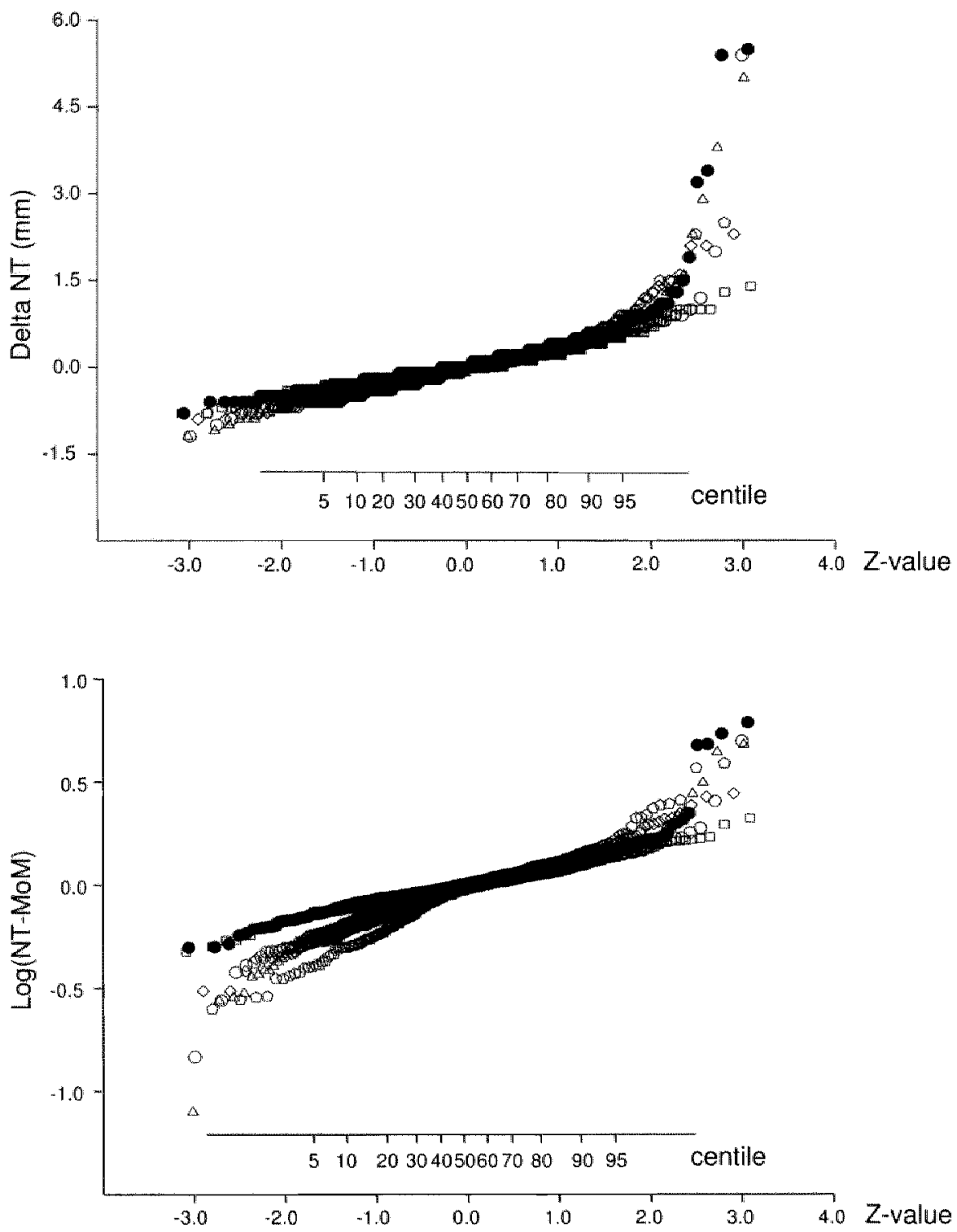
As a consequence, our first trimester combined screening algorithm was less efficient to detect $T 21$ affected pregnancies as compared to results of single centre studies, using FMF scanning criteria ". Our results however were very close to those of multicentre studies using FMF scanning criteria or using group- or performer-specific reference values for NT ${ }^{8}$. We have been investigating several pathways to improve the overall screening results of our program. One of these measures was an attempt to increase the number of FMFtrainees in the program ${ }^{9}$. Despite many efforts, the number of FMF-trainees in Flanders, enlisted at the FMF website, has not yet increased and many obstetricians do not intend to participate in the FMF- program for first trimester ultrasound screening and audit. In this study, we evaluated whether an improvement of screening performance could be expected from the introduction of performer-specific instead of FMF-specific NT-medians and from the use of Delta- NT instead of Log NT-MoM values.

Figure 1 shows that the group-specific CRL-related NT-medians of non-FMFtrained ultrasonographers are systematically lower than those of FMF-trainees. This may be the result of the FMF recommendation to use for each pregnancy the largest NT- value measured ${ }^{3}$. When all NT-measurements are expressed in relation to the $\mathbb{F M F}$ reference range (as is currently done) the FMF-trainees will achieve appropriate values for Delta- NT or Log NT-MoM, but the values of the non-FMF-trainees will be inappropriately low. On the other hand, when the population-specific reference range is used to calculate Delta- NT or Log NTMoM, the values of the non-FMF-trainees will be adequate but those of the FMF-trainees will be too high. This finding clearly illustrates the need to use at least two different reference ranges to calculate Delta- NT or Log NT-MoM in our screening program, one for the FMF-trainees and one for the other ultrasonographers.

Figure 1 also shows that FMF-specific NT medians from the FMF-trainees are around 1 at CRL between 50 and $80 \mathrm{~mm}$, and that this is at CRL between 40 and $80 \mathrm{~mm}$ for the population- specific NT medians of the other 
ultrasonographers. This may be the result of our use of unvalidated FMFreference values at $\mathrm{CRL}<45 \mathrm{~mm}$ : as already discussed above, we received from FMF NT-MoM values at CRL $<45 \mathrm{~mm}$, but also a recommendation not to consider these data valid, as FMF has established its reference range at CRL between 45 and $85 \mathrm{~mm}^{3}$. The current fetal aneuploidy screening algorithm, distributed by FMF, does not allow to implement NT-values measured before 11 weeks of gestation, due to which the gestational window for risk calculations is limited to $11-14$ weeks. On the other hand, our observation may also be an illustration of the difficulty to measure NT correctly at early stages of pregnancy. Zoppi et al. recently reported that the accuracy of NTmeasurements, according to FMF criteria, improved with advancing gestation ${ }^{10}$. We already reported that in our database, $12.4 \%$ of NT- measurements were performed at $\mathrm{CRL}<45 \mathrm{~mm}^{\top}$. Our data illustrate that the use of a population-, group- or performer-specific reference range allows to use NT as a screening parameter for fetal aneuploidy before 11 weeks of gestation, which expands the gestational window for first trimester ultrasound screening for chromosomal abnormalities from 11-14 weeks to approximately 10-14 weeks.

The relevance of using performer-specific NT-medians for fetal aneuploidy screening is illustrated in Figures $2 \mathrm{~A}$ and $2 \mathrm{~B}$. For all ultrasonographers, the curves of CRL-related NT-medians are different, both in the group of FMFtrainees as in the group of other ultrasonographers. The inter-individual variation between these curves is less for the FMF-trained ultrasonographers than for the others. As is shown, the curves of 4 of 5 non-FMF-trainees are relatively close to the curves of the FMF-trainees, which indicates that some ultrasonographers may achieve acceptable NT-measurements, even without official accreditation by FMF. Our results may perhaps be biased by our choice of the non-FMF-trained ultrasonographers with the most numerous NTmeasurements in the AML screening program. These ultrasonographers perform ultrasound screening on a regular base and are likely to have developed a specific method for ultrasonic measurement of NT. This may not be true for 
ultrasonographers who perform a lower number of ultrasound scans. The curve of non-FMF-trained ultrasonographer D illustrates that some ultrasonographers may have NT-measurements, which deviate far from the FMF-criteria or from other ultrasonographers. This is likely to be the result of a lack of methodology, and training into ultrasound screening is recommendable for this person. One of the most important effects of ultrasound training is a reduction of inter- and intra-observer variability or standard deviation of NT measurement ". Parameters showing a nartow variation around the median and a small standard deviation are more valuable for screening purposes than parameters with a wide variation and a large standard deviation ${ }^{12}$. The inter-individual variation of NTmeasurements cannot be corrected for by the use of performer-specific NTmedians. However, the impact of sampling variability, such as for measurement of NT, may be less in multiple parameter screening algorithms than in single or dual parameter algorithms

In Figure 3, we compare the performer-specific reference curves of Delta-NT and Log NT-MoM for 5 non-FMF-trained ultrasonographers with the FMFspecific curve of 1 FMF-trainee. Because in the FMF- audit program, the measurements of all 6 FMF-trainees were approved to meet the FMF criteria, and because of the low inter-observer variation between the FMF-trainees, as shown in Figure 2B, we can consider this FMF-specific curve representative for all FMF-trainees in our program and elsewhere. As is illustrated in Figure 3, the performer-specific curves of non-FMF-trainees are very close to the FMFspecific curves of the FMF-trainee. For the Log NT-MoM probability plots, there is a slight difference between the curves in the lower range of the NTmeasurements $(<\mathrm{P} 40)$, but between the $50^{\text {th }}$ and $95^{\text {th }}$ centile these curves are nearly identical. As there is a direct relationship between the magnitude of NTmeasurement and the likellihood for fetal aneuploidy, the upper range of NTmeasurements is the crucial area with respect to the detection of chromosomal anomalies. For this reason, we think that replacing $\log$ NT-MoM values by Della-NT values in our screening algorithm will be of minor importance 
towards the final screening results of our program. Our data illustrate that the use of performer-specific median NT-values might be considered a valuable alternative for ultrasound training and audit on NT- measurement for those ultrasonographers, who perform a reasonable number of first trimester ultrasound scans.

We conclude from this study that the introduction of performer-specific reference values for NT-measurements in our screening program (1) allows to expand the window for ultrasound screening to gestations less than 11 weeks, and (2) achieves nearly identical distribution curves for Delta-NT and Log NTMoM between the $50^{\text {th }}$ and $95^{\text {th }}$ percentile for both FMF-trainees and nonFMF-trained ultrasonographers.

\subsection{Acknowledgements.}

We thank Prof. N. Wald of the Wolfson Institute of Preventive Medicine, Barts and The London, Queen Mary's School of Medicine and Dentistry, London U.K., for his kind help and advice in this study. We also thank the obstetricians in Flanders, for their appreciated efforts to register pregnancy outcome in the AML- database of fetal aneuploidy screening.

References

Cuckle, $\mathrm{H}$. and Wald,N. (2000) Priciples of screening: tests using single markers. In Antenatal d Neonatal screening. Wald, N. and Leck,I. (eds.) Oxford: Oxford University Press, pp. 3-22.

Gyselaers, W., De Catte, L., Witters, I., De Jonge, E., Ombelet, W., and Nijhuis, J. (2005a) Survey on the future organisation of first trimester ultrasound screening in Flanders, Belgium. Prenat Diagn 25: 518-519.

Gyselaers, W., Vereecken, A., Van Herck. E., Straetmans, D., De Jonge, E., Ombelet, W., and Nijhuis, J. (2004a) Audit on nuchal translucency thickness measurements in Flanders, Belgium: a plea for methodological standardization. Ultrasownd Obstet Gynecol 24: $511-515$.

Gyselaers, W., Vereecken, A., Van Herck, E., Straetmans, D., De Jonge, E., Ombelet, W., and Nijhuis, J. (2005b) Population screening for fetal trisomy $2 \mathrm{l}$ : easy access to screening should be balanced against a uniform ultrasound protocol. Prenat Diagn (in press). 
Gyselaers, W., Vereeken, A., Van Herck, E., Straetmans, D., Martens, G., De Jonge, E., Ombelet, W., and Nijhuis, J. (2004b) Screening for trisomy 21 in Flanders: a 10-years review of 40490 pregnancies screened by maternal serum. Ew J Obstet Gynecol Reprod Biol 115: 185-189.

Logghe, H., Cuckle, H., and Sehmi, I. (2003) Centre-specific ultrasound nuchal transincency medians needed for Down syndrome screening. Prenat Diagn 23: 389-392.

Nicolaides, K. H., Heath, V., and Cicero, S. (2002) Increased fetal nuchal translucency at 1 I-14 weeks. Prenat Diagn 22: 308-315.

Pajkrt, E., Mol, B. W., Boer, K., Drogtrop, A. P., Bossuyt, P. M., and Bilardo, C. M. (2000) Intraand interoperator repeatability of the nuchal translucency measurement. Uhrasound Obstet Gynecol 15: 297-301.

Pandya, P. P., Snijders, R. J., Johnson, S. P., De Lourdes, Brizot M., and Nicolaides, K. H. (1995) Screening for fetal trisomies by maternal age and fetal nuchal translucency thickness at 10 to 14 weeks of gestation. Br J Obster Gynaecol 102: 957-962.

Wald,N. and Hackshaw, A. (2000) Tests using multiple markers. In Antenatal and neontatal screening. Wald,N., Leck,l, and . (eds.) Oxford: Oxford University Press, pp. 23-57.

Wald, N. J., Rodeck, C., Hackshaw, A. K., Walters, J., Chitty, L., and Mackinson, A. M. (2003) First and second trimester antenatal screening for Down's syndrome: the results of the Serum, Urine and Ultrasound Screening Study (SURUSS). JMed Screen 10: 56-104.

Zoppi, M. A., Ibba, R. M., Floris, M., Manca, F., Axiana, C., and Monni, G. (2005) Nuchal translucency measurement at different crown-rump lengths along the 10 - to 14 -week period for Down syndrome screening. Prenat Diagn 25: 411-416. 
Chapter 10

\section{Fetal aneuploidy screening}

practice in Flanders and Belgium

Gyselaers WJA, Vereecken AJ, Nijhuis JG. In. Slager E, Fauser BCIM, van Geijn HP, Brölman HAM, Vervest HAM (eds). Gynaecology, obstetrics and reproductive medicine in daily practice. Intemanional Congres, Series 2005; 1279: $228-233$. 
162 


\section{Summary}

The current situation of antenatal fetal aneuploidy screening in Belgium is reviewed for the provision of screening service, the performance and results of different screening methods and the registration of perinatal data on congenital malformations. Some options are discussed towards improvement of future screening practice in the Belgian pregnant population. 


\subsection{Introduction.}

Offering amniocentesis (AC) or chorionic villus sampling (CVS) to pregnant women aged 35 years or more was the first method to screen for fetal aneuploidy in Belgium. Since 1990, an increasing number of these invasive procedures was observed (Vamos et al., 1997). Both in Liège and Louvain, the detection of fetal trisomy 21 (T21) increased significantly after the introduction of second trimester maternal serum screening (Verloes et al., 2001; Witters et al., 2002). In 1996, the ultrasonic measurement of fetal nuchal translucency (NT) in the first trimester of pregnancy was introduced as a new method to screen for fetal aneuploidy. Soon, this parameter was combined with second trimester maternal serum and later also with first trimester maternal serum parameters (Gyselaers et al., 2004d). As there are no guidelines on fetal aneuploidy screening in Belgium, today these different screening methods are all used randomly.

In this paper, we review the Belgian situation for (1) provision of fetal aneuploidy screening service, (2) performance and results of different screening methods and (3) registration of perinatal data on fetal aneuploidy. Some options are discussed towards optimalisation of future screening practice in the Belgian pregnant population.

\subsection{Provision of fetal aneuploidy screening service.}

The evolution between 1995 and 2001 of maternal serum screening in Belgium is shown in Table 1. These data are from the register of the National Institute for Health Insurance (RIZIV) and the Christian Health Insurance Company (CM). The data from both registers are very similar for the years 2000 and 2001. As is shown, maternal serum screening was performed in $42.5 \%$ of all pregnancies $>$ 12 weeks in 1995 and this fraction increased to $55.1 \%$ in 2001 . From these data, it is not clear whether women, who had no maternal serum screening, had no access to maternal serum screening programs or simply denied fetal aneuploidy screening. 
Table 10.1. The numbers of pregnancies $>12$ weeks and matemal serum screening tests in Belgium between 1995 and 2001 , as registered by the National Institute for Health imsurance (RIZV) and the Christian Healn Insurance Company (CM). Tho pregnancies registered by $\mathrm{CM}$ are 40-50\% of all Belgian pregnancies, which are in the RIZTV register; RVZV did not register matemal serum screening before 1999.

$\begin{array}{lllllll}1995 & 1996 & 1997 & 1998 & 1999 & 2000 & 2001\end{array}$

\begin{tabular}{ccccccccc} 
RIZIV Pregn > 12w & 105815 & 110156 & 108279 & 107408 & 108685 & 110239 & 112616 \\
& Mat serum & & & & & 17910 & 59230 & 62437 \\
$\%$ & & & & & 16.5 & 53.7 & 55.4 \\
& & & & & & & & \\
CM & Pregn > 12 w 50791 & 51781 & 50061 & 49441 & 48502 & 47882 & 45877 \\
& Mat serum & 21575 & 24644 & 24547 & 24109 & 25093 & 25799 & 25257 \\
& $\%$ & 42.5 & 47.6 & 49.0 & 48.8 & 51.7 & 53.9 & 55.1 \\
\hline
\end{tabular}

Over $95 \%$ of antenatal care in Flanders is provided by obstetricians (Kind en Gezin, 2003). As most obstetricians perform obstetric ultrasound as a routine antenatal procedure, refunded by the National Health Insurance System at 3 examinations per pregnancy, pregnant women in Flanders do have easy access to fetal anomaly screening by ultrasound. Few obstetricians however comply to specific programs for training and audit on first trimester ultrasound screening, such as the program provided by the Fetal Medicine Foundation (FMF). Of a total number of nearly 500 obstetricians, enregistered as a member of the Flemish Society for Obstetricians and Gynecologists, less than 20 are enlisted as accredited ultrasonographers on the FMF-website (2004). Only 6 FMF-trainees were present in a group of 264 obstetricians, involved in the fetal aneuploidy screening program organised by the Algemeen Medisch Laboratorium (AML) in Antwerp (Gyselaers et al., 2004b). In this screening program, more than $12 \%$ of NT measurements were taken outside the FMF-recommended gestational window of 11 to 14 weeks. These data indicate that currently Flemish pregnant women do have easy access to ultrasound screening, but only a minority is examined according to one specific ultrasound screening program. This may be 
another illustration of poor access for pregnant women to stringent ultrasound screening for fetal aneuploidy, as has been reported elsewhere (Egan et al., 2002; Whittle, 2001).

\subsection{Performance and results of different screening methods.}

\subsubsection{Screening at adwanced maternal age.}

In 1994, it was reported that maternal serum screening enhances the reduction of the number of primary invasive procedures in pregnancies at advanced maternal age, without a major reduction of T21 detection rate (DR) (Haddow et $a l ., 1994)$. Both in the first and second trimester, maternal serum screening identified a proportion of women $\geq 35$ years of age whose estimated risk for fetal aneuploidy was less than the risk for fetal loss as a result of invasive testing (Gyselaers et al, 2004c).

In a 10-years review on fetal aneuploidy screening in Flanders, it was observed that the proportion of women $\geq 35$ years, submitted to maternal serum screening, was smaller than expected from the maternal age distribution in the total pregnant population (Gyselaers et al., 2004d). The Centre for Human Genetics in Louvain reported advanced maternal age to be the indication for $35 \%$ of diagnostic invasive procedures in 2001 (Witters, 2002).

In ultrasound screening, it was shown that a small variation of NT measurement, within the $95 \%$ confidence interval for inter-and intra-observer variability (Pajkrt et al., 2000; Pandya et al., 1995), had much more impact on the final screening result of women $\geq 35$ years of age in comparison to younger women (Gyselaers et al., 2004b). This effect was significantly less when other parameters, such as serum parameters, were included in the screening algorithm.

\subsubsection{Second trimester maternal serum screening.}

Since its introduction in 1992, fetal aneuploidy screening with second trimester maternal serum concentrations of $\alpha$-Feto Protein (AFP), total $\beta$-Human 
Chorionic Gonadotropin (HCG) and Estriol $\left(\mathrm{E}_{3}\right)$ has become a well established screening method in Flanders. In a total of 36382 pregnant women, a $69.7 \%$ T21 DR was reported at a $5.5 \%$ false positive rate (FPR) (Gyselaers et al., 2004d). These results were very similar to data from literature (Wald et al., 1997).

\subsubsection{Ultrasonic measurement of fetal nuchal translucency (NT).}

Fetal NT and other ultrasonographically detected soft markers for fetal aneuploidy were the indication for $7.5 \%$ of all diagnostic invasive procedures in 2001 in the Centre for Human Genetics in Louvain (Witters et al., 2002). An audit on 13000 NT measurements in Flanders showed a systematic underestimation in comparison to the FMF reference range (Gyselaers et al., $2004 \mathrm{~b})$. Failure to comply to the guidelines for ultrasonic measurement of NT, as recommended by FMF (Nicolaides et al., 2002), was considered the most important explanation for this observation. The T21 DR at 5\% FPR of this nonstringent ultrasound screening was $40 \%$ (Gyselaers et al., 2004a), which was much lower than the $75-80 \%$ DR of stringent ultrasound screening, as reviewed in literature (Brigatti and Malone, 2004). However, this 40\% DR was similar to the performance of other single parameter screening methods, such as AFP, $\mathrm{HCG}$ or $\mathrm{E}_{3}$ (Wald et al., 1997). Therefore, non-stringent measurement of NT may still be a valuable parameter in multiple parameter screening, as in combined or integrated screening algorithms (Wald et al., 2003).

\subsubsection{Second trimester maternal serum screening and NT.}

In the AML screening program, the T21 DR was lower for second trimester maternal serum screening with NT than without NT (Gyselaers et al., 2004d). In the population screened with serum and NT, the mean maternal age was higher and the prevalence of $T 21$ was lower than in the population screened with serum only. It was concluded that a two step sequential screening practice was performed, with first trimester screening by NT and subsequent referral of pregnancies with positive screening results for invasive testing, leaving only the pregnancies with negative screening results for further second trimester 
maternal serum screening. The FPR in this study however was much higher than expected from literature (Hackshaw and Wald, 2001). In second trimester screening after prior first trimester screening, it was recommended to adjust the individual's back-ground risk for correct risk estimation (Hackshaw and Wald, 2001). This adjustment was not performed in the AML-study, as the population studied was a mixture of sequentially and non-sequentially screened pregnancies. However, the AML data clearly illustrate that multiple step parameter sampling may enhance multiple step risk calculations, with the subsequent risk of higher FPR rates than single step integrated risk calculations (Wald et $a l_{n}, 1997$ ). A solution to this problem of the AML screening program is urgently needed when integrated screening with first and second trimester parameters is to be introduced in Flanders.

\subsubsection{First trimester maternal serum screening and NT (combined screening).}

Despite the poor performance of screening by maternal age and NT in the AML screening program, the T21 DR was $81.2 \%$ at $5 \%$ FPR for combined first trimester screening with maternal age, NT and serum concentrations of Pregnancy Associated Plasma Protein-A (PAPP-A) and free B-HCG (Gyselaers et al., 2004a). These figures were similar to the results reported from other multicentre studies (Muller et al., 2003; Wald et al., 2003; Wapner et al., 2003). This finding clearly illustrates an advantage of multiple parameter screening algorithms over single or dual parameter algorithms, as is already discussed above.

\subsection{Registration of perinatal data on fetal aneuploidy.}

In Belgium, information on perinatal activities is registered in several databases: RIZIV, CM and other health insurance companies, Studiecentrum voor Perinatale Epidemiologie (SPE), Office de la Naissance et de l'Enfance (ONE), Kind \& Gezin, Eurocat. However, none of these databases registers the whole spectrum of fetal aneuploidy screening into detail. Only the Eurocat register 
provides information on the antenatal detection and terminations of pregnancy for fetal $\mathrm{T} 21$ and other chromosomal abnormalities in two Belgian regions: Antwerp and Hainaut (2003)). Between 1992 and 2001, an increasing trend was found in both regions for terminations of T21-affected pregnancies and for the overall prevalence of $\mathrm{T} 21$, but an opposite evolution was found for the live birth prevalence of T21. This T21 live birth prevalence increased in Antwerp and decreased in Hainaut. It is very difficult to explain these observations, because of the lack of information on the population characteristics in both regions and on the application and results of different fetal aneuploidy screening methods. This clearly illustrates the need for a national perinatal database on fetal aneuploidy screening in Belgium, in order to understand the natural course of affected pregnancies and the impact of antenatal screening and interventions. Without such register, it will be very difficult or perhaps even impossible to perform any evaluation and quality control of fetal aneuploidy screening practices in the country.

\subsection{Optimisation of future screening practice in Belgium.}

Several measures may improve the results of current fetal aneuploidy screening practice in Belgium.

A survey on the future organisation of ultrasound screening in all 75 obstetric clinics in Flanders showed that the majority of the chief-obstetricians considered ultrasound screening for fetal aneuploidy a routine antenatal service, to be available in every clinic (Gyselaers et al., 2005). In this study, most clinicians requested further training in ultrasound methodology and agreed for audit and quality control of their own performance. The Specialist Centres for Obstetric Ultrasound in the country have already initiated the organisation of training courses on first trimester ultrasound screening for Flemish obstetricians, in order to increase the provision of high quality first trimester ultrasound screening. 
As both first and second trimester screening methods are available in Belgium, it should be possible to integrate all parameters into one single algorithm, which may increase DR and reduce FPR (Wald et al, 2003). The introduction of the ultrasonic evaluation of the fetal nasal bone and other parameters may further improve the overall screening performance (Cicero et al., 2003).

The measured values of all parameters used in fetal. aneuploidy screening usually are expressed as a Multiple of the Median at every specific stage of gestation (MoM). To calculate these MoM-values, the application of population-, centre- or performer-specific reference values is considered the best option for all parameters in the screening algorithms (Logghe et al., 2003; Wald et al., 2003). Today, the AML algorithm still implements NT-MoM values relative to the FMF reference range (Gyselaers et al., 2004b). The application of population specific reference values in this algorithm is currently under investigation.

As is explained above, a Belgian Register of Congenital Anomalies may provide all relevant information to audit the performance of different screening methods and to guarantee high quality screening.

The introduction of contingent testing may also improve the access for pregnant women to first trimester screening in Belgium [27]. In this system, maternal serum screening is offered to all pregnant women, and NT measurements are only performed in those pregnancies considered at higher risk. Serum sampling can be performed in all first line antenatal clinics of obstetricians, general practitioners and midwives, and the subsequent NT measurements can be performed by trained ultrasonographers. This system of fetal aneuploidy screening in two levels may offer a rational and practical solution to the problem "how to offer the highest possible detection rate at the lowest possible false positive rate to the largest possible fraction of the pregnant population?" 


\section{References}

Brigatti, K. W. and Malone, F. D. (2004) First-trimester screening for aneuploidy. Obstet Gynecol Clin North Am 31: $1-20$

Cicero, S., Bindra, R., Rembouskos, G., Spencer, K., and Nicolaides, K. H. (2003) Integrated ultrasound and biochemical screening for trisomy $2 \mathrm{I}$ using fetal nuchal translucency, absent tetal nasal bone, free beta-hCG and PAPP-A at 11. to 14 weeks. Prenat Diagn 23: 306-310

Egan, J. F., Kaminsky, L. M., DeRoche, M. E., Barsoom, M. J., Borgida, A. F., and Benn, P. A. (2002) Antenatal Down syndrome screening in the United States in 2001: a survey of maternalfetal medicine specialists. An J Obster Gynecol 187: 1230-1234.

Gyselaers, W., De Catte, L., Witters, I., De Jonge, E., Ombelet, W., and Nijhuis, J. (2005) Survey on the future organisation of first trimester ultrasound screening in Flanders, Belgitum. Prenat Diagn 25: 518-519.

Gyselaers, W., Vereecken, A., Van Herck, E., Straemans, D., De Jonge, E., Ombelet, W., and Nijhuis, J. (2004a) Audit on fetal Down's Syndrome screening in a Belgian population between 1992 and 2004. J Matern Fetal Neonatal Med 16 (suppl I): 44.

Gyselaers, W., Vereecken, A., Van Herck, E., Straetmans, D., De Jonge, E., Ombelet, W., and Nijhuis, J. (2004b) Audit on nuchal translucency thickness measurements in Flanders, Belgium: a plea for methodological standardization. Ultrasound Obstet Gynecol 24: $511-515$.

Gyselaers, W., Vereecken, A., Van Herck, E., Straetmans, D, De Jonge, E., Ombelet, W., and Nijhuis, J. (2004c) Single step maternal serum screening for fetal trisomy 21 in the era of combined or integrated screening. Gynecol Obster Invest 58: 221-224.

Gyselaers, W., Vereecken, A., Van Herck, E., Straetmans, D., Martens, G., De Jonge, E., Ombelet, W., and Nijhuis, J. (2004d) Screening for trisomy 21 in Flanders: a 10-years rewiew of 40490 pregnancies screened by maternal serum. Eur J Obstet Gynecol Reprod Biol 115: 185-189.

Hackshaw, A. K. and Wald, N. J. (2001) Inaccuratte estimation of risk in second trimester serum screening for Down syndrome among women who have already had first trimester screening. Prenat Diagn 21: $741-746$.

Haddow, J. E., Palomaki, G. E., Knight, G. J., Cunningham, G. C., Lustig, L., S., and Boyd, P. A. (21-4-1994) Reducing the need for amniocentesis in women 35 years of age or older with serum markers for screening. $N$ Engl $/$ Med 330 : 1114-1118.

Kind en Gezin. The child in Flanders, 2002. Hup:/www.kindengexin.be

Logghe. H., Cuckle, H., and Sehmi, I. (2003) Centre-specific ultrasound nuchal translucency medians needed for Down syndrome screening. Prenai Diagn 23; 389-392.

Muller, F., Benattar, C., Audibert, F., Roussel N., Dreux, S., and Cuckle, H. (2003) Firsttrimester screening for Down syndrome in France oombining fetal nuchal translucency measurement and biochemical markers. Prenat Diagn 23: 833-836. 
Nicolandes, K. H., Heath, V., and Ciceno, S. (2002) Increased fetal nuchal translucency at $1.1-14$ weeks. Prenal Diagn 22: 308-315.

Pajkrt, E, Mol, B. W., Boer, K., Drogtrop, A. P., Bossuyt, P. M., and Bilardo, C. M. (2000) Intraand interoperator repeatability of the nuchal translucency measurement. Ultrasound Obstet Gynecol 1.5: 297-301.

Pandya, P. P., Altman, D. G., Brizot, M. L., Pettersen, H., and Nicolaides, K. H. (1995) Repeatability of measurement of fetal nuchal translucency thickness. Ultrasound Obstet Gymecol 5: $334-337$.

Vamos, E., Vandenberghe, K., and Cassiman, J. J. (1997) Prenatal diagnosis in Belgium. Eur J Hum Gener 5 Suppl 1: $7-13$.

Verloes, A., Gillerol, Y., Van Maldergem, L., Schoos, R., Herens, C., Jamar, M., Dideberg, V., Lesenfants, $S$., and Koulischer, L. (2001) Major decrease in the incidence of trisomy 21 at birth in south Belgium: mass impact of triple test? Eur J Hum Genet 9: 1 4.

Wald, N. J., Kennard, A., Hackshaw, A, and McGuire, A. (1997) Antenatal screening for Down's syndrome. J Med Sicreen 4: 181-246.

Wald, N. J., Rodeck, C., Hackshaw, A. K., Walters, J., Chitty, L., and Mackinson, A. M. (2003) First and second trimester antenatal screening for Down's syndrome: the results of the Serum, Urine and Ultrasound Screening Study (SURUSS). J Med Screen 10; 56-104.

Wapner, R., Thom, E., Simpson, J. L., Pergament, E., Silver, R., Filkins, K., Platt, L., Mahoney, M., Johnson, A., Hogge, W. A., Willson, R. D., Mohide, P., Hershey, D., Krantz, D., Zachary J., Snijders, R., Greene, N., Sabbagha, R., MacGregor, S., Hill, L., Gagnon, A., Hallahan, T., and Jackson, L. (9-10-2003) First-trimester screening for trisomies 21 and 18. N Engl J Med 349: 1405-1413.

Whittle, M. (2001) Down's syndrome screening: where to now? BJOG 108: 559-561.

Witters, 1. Prenatal diagnosis of fetal malformations and chromosomal anomalies: a multidisciplinary appronch. 7-158.2002. Catholic University Louvain. 2002.

Witters, I., Legius, E., Matthijs, G., and Fryns, J. P. (2002) Prenatal diagnosis of trisony 21 between 1991 and 1999 in the Leuven Centre for Human Genetics: effect of triple test screening. Genet Couns 13: 199-202. 
Chapter 11

\section{General discussion and future}

\section{perspectives}


During the progress of this thesis, there has been an evolution in the way of handling, interpreting and presenting the data as a result of a learning process. In the first publication (Chapter 4), we struggled with the large number of screened pregnancies, of which information on the clinical outcome was lacking. In that paper, we presented data of those pregnancies with complete follow up data, which was only $51.7 \%$ of the total population screened. For the same reason, we used in the next publications (Chapter $5 \& 6$ ) the definitions "Screen Positive Rate" instead of "False Positive Rate". We learned how to evaluate whether all T21 affected pregnancies were reported to the laboratory at the "Intensive Course on Antenatal Screening for Fetal Down's Syndrome", held at the Wolfson Institute of Preventive Medicine in May 2004. It was not until the AML methods of registering and risk calculating were fully validated (Chapter 3) that the data interpretation wasn't troubled anymore by the inadequate communication between the clinicians and the laboratory. The practice of sequential screening (Chapter 3) and the impact of ultrasound screening (Chapter $3,6 \& 8$ ) on the final screening results was only understood after the audit on nuchal translucency (NT) thickness measurements (Chapter 6). After that, recommendations could be made towards improvement of ultrasound screening methods (Chapter 7), improvement of the screening algorithms (Chapter 9) and improvement of fetal aneuploidy screening practice in general in Flanders (Chapter 8 \& 10).

From the data presented in the previous chapters, it is possible to answer the four basic questions made at the onset of this thesis :

\section{Which are the different fetal aneuploidy screening methods available in} Flanders / Belgium and do they have the same sensitivity (detection rate) and specificity (false positive rate) as reported in literature?

Today, many fetal aneuploidy screening methods are used in Flanders: (1) primary invasive testing for maternal age $\geq 35$ years (Chapter 4), (2) second trimester maternal serum screening with AFP, $\mathrm{HCG}$ and $\mathrm{uE}_{3}$ (Chapter 4 \& 5), 
(3) first trimester ultrasound screening with nuchal translucency (NT) measurement, (4) first trimester combined screening with NT and PAPP-A and FB-HCG (Chapter $6 \& 8$ ) and (5) second trimester combined screening where NT is combined with (or integrated in) second trimester triple screening (Chapter 4). As there are no guidelines on fetal aneuploidy screening in Flanders, these methods are all used randomly to screen the population. This observation may be an illustration of the flexibility needed to screen a population for fetal aneuploidy. Every screening test has practical limitations, such as the need for an optimal gestational window for parameter sampling, due to which it is impossible to present one single screening method to every pregnant woman. In fact, population screening for fetal aneuploidy requires a pallet of screening tests to meet all interfering factors caused by the divergent availability of prenatal care and the pick up by the pregnant women.

Today, the second trimester quadruple test or the integrated test with PAPP-A, NT and second trimester serum parameters are not (yet) used in daily clinical practice in Belgium, so in this thesis no data can be presented on these screening methods.

- The performance of both first trimester serum screening and second trimester triple screening, presented in this thesis, is comparable to the results reported in literature: the T21 DR is around $65 \%$ at $5 \%$ FPR (Chapter 4 \& 5).

- From the clinical point of view, we should consider three different screening methods, currently used in Flanders, in which ultrasound parameters are involved: (1) screening by ultrasound parameters only, (2) screening by ultrasound and first trimester maternal serum parameters and (3) screening by ultrasound and second trimester maternal serum parameters. In this thesis, data are presented on the combined screening methods (2) and (3), as all the screening tests were performed in a medical laboratory, where the serum samples were analysed. Due to this, we have no data on genuine ultrasound screening 
only. The performance of the ultrasound component in the AML first trimester combined algorithm is clearly different from what would be expected from literature: at $5 \%$ FPR a DR of only $42 \%$ was found, which was much less than the $75 \%$ reviewed in literature (Chapter 8 ). This was considered the result of a systematic underestimation of NT thickness by the non-FMF-trained ultrasonographers, compared to the FMF-trainees whose measurements were very close to the FMFreference values (Chapter 6). However, the proof of this is not in this thesis: because of insufficient sample size we are unable to compare screening results between FMF-trained and -untrained ultrasonographers. The ultrasound screening results might improve after ultrasound training and quality control, which has already been initiated in Flanders (Chapter 7). This is particularly relevant when new ultrasound parameters, such as the fetal nasal bone, are to be introduced in the screening algorithms. However, today it is already clear that not every obstetrician, involved in fetal aneuploidy screening in Flanders, is willing to participate in any training and audit program. Therefore it seems unlikely that in the future every performer- specific NT distribution curve in the country will fully comply to the FMF criteria, even when training and audit are installed and active. To achieve the best possible results from ultrasound screening, clearly more interventions will be needed, such as (1) the reorganisation of screening activities, due to which ultrasound screening should be limited to only those ultrasonographers participating in an audit program, or (2) the introduction of population-, performer- or group- specific NT medians into the screening algorithms (Chapter 9). The use of performer- or group- specific NT medians in Flanders both combines an easy access to screening (Chapter 8) with a good efficiency from ultrasound parameters. The interventions to optimise the current screening 
performance in the AML screening program will be discussed further in this chapter.

An important finding from our audit is that, despite the poor NT performance, the results of the AML first trimester combined algorithm were comparable to other multicenter studies reported in literature. In the paper (Chapter 8), it is explained that the high relative weight of maternal serum screening compared to ultrasound screening in the AML algorithm may account for this result. In Chapter 6, we illustrated the relevance of combining ultrasound and maternal serum parameters: a $\pm 0.1 \mathrm{~mm}$ deviation of NT measurement had much less impact on the final screening results (SPR) when maternal serum parameters were included in the algorithm, compared to ultrasound screening only.

- The interpretation of combined (integrated) screening with first trimester NT measurement and second trimester triple test in our screening program was clearly more difficult. In Chapter 4 , we report a different performance of triple screening after the introduction of NT measurements in 1998. This was considered the result of a sequential screening practice, one step in the first trimester (NT) and a separate step in the second trimester (triple test). This interpretation was wrong: it is clear now that the results presented in chapter 4 were related to the small sample size of the population studied. However, since 1999 sequential screening actually wàs performed in Flanders as is illustrated by the large fraction of missing $\mathrm{T} 21$ affected pregnancies in the population screened by second trimester triple test (Chapter 3, Table 4). Many obstetricians indeed admitted to practice a sequential screening policy, when they were asked about their fetal aneuploidy screening attitudes. These findings will be presented in a new manuscript, which is currently in preparation. The implications of sequential screening in Flanders and possible solutions are discussed further in this chapter. 


\section{Can the optimal fetal aneuploidy screening method in Flanders / Belgium}

be identified, from the point of view of the individual patient and the population?

From all screening methods currently available to an individual pregnant woman in Flanders, combined first trimester screening offers the highest detection rate (DR) at the lowest false positive rate (FPR). As explained in Chapter 8 , the risk calculated from the first trimester combined algorithm correlates well with the observed prevalence of trisomy 21 (T21), and therefore this method is appropriate to counsel every individual patient. The performance of this screening method and the possible interventions for optimisation of the ultrasound screening component have been discussed above.

In Chapter 8, we showed that the introduction of the first trimester combined algorithm into population screening for fetal aneuploidy is much easier with a non specified ultrasound methodology than with the FMF ultrasound screening criteria (Chapter 8). From the point of view of the population in the AML screening program, first trimester combined screening with non specified ultrasound methods is currently the best screening method as it is responsible for the majority of T21 detections (Chapter 8), despite its lower performance than reported from the FMF single centre studies. However, as is also explained in Chapter 8, the performance of the FMF screening methods in multicentre studies is also less than in single centre studies, which illustrates that it is still to be proven that the general introduction of FMF scanning criteria into population screening for fetal aneuploidy is realistic and feasible.

Although first trimester combined screening is currently the best screening method in the AML screening program for both the individual patient and the population, its performance may benefit from several quality improving measures. However, it is expected that much better screening results will be achieved after (1) introduction of the integrated first and second trimester screening test and/or (2) reorganisation of screening services in a system of contingent screening. This will also be discussed further in this chapter. 
3. Is it possible to identify any strategies, which improve the detection rate and reduce the false positive rate and subsequent invasive testing of unaffected pregnancies in Flanders / Belgium?

From the data presented in this thesis, there may be two important measures towards reduction of invasive testing in unaffected pregnancies in Flanders. (1) Amniocenteses or chorionic villus sampling in unaffected pregnancies at maternal age $\geq 35$ years can be reduced significantly, when they would only be performed for prior positive maternal serum and/or ultrasound screening (Chapter 4 \& 5). As the impact of small deviations of NT measurement is most relevant to the screening results of women at advanced maternal age, accuracy in ultrasound screening methods is very important at $\geq 35$ years of age (Chapter 6). (2) As is explained in Chapter $3 \& 4$, a policy of two step sequential screening is practised in Flanders, in which second trimester maternal serum screening is only performed after normal first trimester NT measurement. It has been reported that this sequential screening practice results in higher FPR than single step combined screening and therefore should be abandoned. An elegant way to increase detection rate and reduce false positive rate of future fetal aneuploidy screening in Flanders is the integration of first and second trimester parameters in the so-called integrated test. Efforts were made to present the principle of integrated screening to all obstetricians involved in fetal aneuploidy screening in Flanders. Apart from the overall improvement of screening performance, there are a few specific reasons why current fetal aneuploidy screening practice in Flanders and Belgium may benefit from integrated screening:

1. Currently, ultrasound screening is the weak component in the AML screening algorithm (Chapter 6,8). The impact of suboptimal parameter sampling by ultrasound will be less when a higher number of serum parameters is used for risk calculations. 
2. Today, pregnant women in Belgium have easier access to high quality maternal serum screening than to high quality ultrasound screening (Chapter 8).

However, when integrated screening is to be introduced in Belgium, the poor communication between the level of parameter sampling (obstetricians) and parameter handling (laboratory) has to improve. As explained in Chapters 4 and 10, the AML register contains a large proportion of pregnancies with missing data on pregnancy outcome or without information on prior steps in the screening process (mostly ultrasound screening). This problem will be discussed further in this chapter.

Another method which might improve the current screening results in Flanders or Belgium is the reorganisation of services in a system of contingent screening. First trimester maternal serum screening can easily be offered to all pregnant women willing to participate in the screening process (Chapter 8). From this, pregnancies can be identified with (1) a very high risk for $T 21$, justifying immediate diagnostic invasive testing, (2) a very low risk, justifying an interruption of the screening process because of the low probability that the result will turn positive after adding more parameters and (3) an intermediate risk, which justifies further (integrated) screening with ultrasound examinations, performed by trained ultrasonographers only, and/or second trimester maternal serum parameters.

The measures to improve results from ultrasound screening activities in Flanders have already been discussed above.

\section{Is the current registration and follow up of screening practice in Flanders /} Belgium sufficient to guarantee a satisfactory audit and quality control of performance, and to guide future optimisation of screening?

In this thesis, one of the most difficult problems to tackle was the lack of information on (1) the outcome of all screened pregnancies and on (2) all steps of the screening process. Collaborating obstetricians reported these items. 
voluntarily to the laboratory, but the information was often incomplete (Chapter $3 \& 4)$. For this reason, the AML methods of registry had to be validated as explained in the Materials and Methods section (Chapter 3). This validation would not have been necessary with a decent register on all steps in the screening process. The lack of valid information from the AML sequential screening program, combining (integrating) first trimester ultrasound and second trimester maternal serum parameters, clearly illustrates the need for a decent register towards audit, quality control and optimisation of future screening. This problem needs solving when integrated screening is to be introduced in Flanders, as data on many parameters will be used, often sampled at different stages of gestation. In order to understand differences in the epidemiology of fetal aneuploidy between different regions in Belgium, as is documented in the Eurocat register (Chapter 1.0), it is important that this registry should be centralised or that the methodology should be similar for all regions.

Following the report on the cost-beneficial effects of overall prenatal genetic testing in the population, the ultimate goal for every fetal aneuploidy screening program in the future should be the ability to offer safe genetic diagnostic tests to every pregnant women without putting her pregnancy at risk. For as long as these safe tests are not yet available and the current invasive procedures are troubled with iatrogenic losses of unaffected pregnancies, the direction for fetal aneuploidy screening practice in the future should be towards the most sensitive and specific screening methods at acceptable costs for the society. Some strategies to achieve this goal have been discussed extensively in this thesis. Above all however, a change in the attitude of Belgian doctors and patients may be necessary. Traditionally, Belgian medical health care has been very liberal. This is symbolised by the free right for both doctors and patients to choose a test or treatment. This right has always been considered superior to the economic aspects of health care. In this system however, introspection and reflection on daily clinical practice was not always wanted by many of the 
health care providers, as this would limit the free traffic of patients and medical activities. This thesis as a whole is an illustration of the relevance to audit daily clinical practice: the strengths and weaknesses of current screening activities in Flanders are identified and strategies towards further improvement of care are proposed. The economic consequences of audit have not been discussed in this thesis, as this on itself may be subject of another thesis. Finding the right balance between the freedom of choice for patients and doctors, the quality of care and the costs for the society will be one of the major challenges towards the further development of fetal aneuploidy screening practice in Belgium.

Finally, none of the current screening methods offers $100 \%$ detection of fetal aneuploidy. This implies that there will always be births of handicapped children due to chromosomal abnormalities, which are missed by any mode of screening. Every organisation of fetal aneuploidy screening therefore is considered incomplete or even negligent when these missed cases should not be taken into account. Together with the affected neonates, born from mothers who refused screening, the affected births after failed screening utterly deserve a decent medical, psychological and social support. Every society, which is willing to pay for activities to reduce the birth of handicapped children, automatically forces itself into the organisation of supporting system for those missed or accepted cases. For Flanders, these supporting systems should include the availability of professional counselling during pregnancy in every clinic where screening is offered, appropriate psychological and social support at birth for parents and their families, and measures to reduce the waiting lists at institutions for mental illness or to increase the facilities towards integration of handicapped people into society. These social aspects may be subject of again another thesis. 
Chapter 12

\section{Summary}


Both in Belgium and The Netherlands (and probably also in many other countries), practical limitations obstruct or delay an efficient introduction of new technologies into population screening for fetal aneuploidy. These limitations are mainly related to the organisation of health care in these countries. This thesis is an audit on the daily clinical practice of fetal aneuploidy screening in Flanders, Belgium, using the screening program of Algemeen Medisch Laboratorium in Antwerp as an example. It illustrates the relevance to evaluate the quality and performance of the current screening methods, and to identify their strengths and weaknesses. This audit is an essential step towards optimisation and future organisation of prenatal screening.

In Chapter 1, the history of fetal aneuploidy screening is briefly discussed, and the different screening methods are presented: invasive testing for advanced maternal age, maternal serum screening and ultrasound screening. The risks of amniocentesis and chorionic villus sampling are compared. The methodology, performance and clinical application of maternal serum screening and ultrasound screening are discussed and compared, and it is explained how both methods may efficiently reduce invasive procedures in unaffected pregnancies at advanced maternal age. Some differences in the organisation of health care and fetal aneuploidy screening in Belgium and The Netherlands are discussed.

Chapter 2 presents the evolution of maternal serum screening and invasive testing in Belgium between 1995 and 2001, using data from the National Institute for Health Insurance (RIZIV) and of the Health Insurance Fund CM. It is illustrated that the rise of invasive testing in this 7-years period was mainly the result of an increasing number of invasive testing without prior serum screening at advanced maternal age. This observation was clearly different than expected from the data presented in Chapter $\mathbb{1}$, and initiated the onset of this thesis. We set out to investigate four basic questions on fetal aneuploidy screening practice in Flanders / Belgium: 
5. Which are the different fetal aneuploidy screening methods available in Flanders / Belgium and do they have the same sensitivity (detection rate) and specificity (false positive rate) as reported in literature?

6. Can the optimal fetal aneuploidy screening method in Flanders / Belgium be identified, from the point of view of:

- the individual patient

- the population

7. Is it possible to identify any strategies, which improve the detection rate and reduce the false positive rate and subsequent invasive testing of unaffected pregnancies in Flanders / Belgium?

8. Is the current registration and follow up of screening practice in Flanders / Belgium sufficient to guarantee a satisfactory audit and quality control of performance, and to guide future optimisation of screening?

Chapter 3 explains some basic principles of fetal aneuploidy screening, which were relevant to develop the algorithms for the fetal aneuploidy screening program of Algemeen Medisch Laboratorium (AML) in Antwerp, Belgium. The AML register is one of two published databases on population screening for fetal aneuploidy in Belgium. The methodology of this screening program is presented. It is explained how these methods were validated and how quality control and optimisation of the screening program was performed.

Chapter 4 reviews a 10- years period of maternal serum screening: the performance of second trimester maternal serum screening was comparable to data from literature, but the proportion of women screened at maternal age $\geq 35$ years was less than expected from the maternal age distribution in the total population of pregnant women in Flanders. This observation is considered the result of primary invasive testing in a considerable number of pregnancies at advanced maternal age. The interpretation of the results of second trimester 
triple screening, after the introduction of nuchal translucency measurement (NT) as a screening parameter, was difficult due to small sample size. After 1999, the prevalence of trisomy $2 \mathbb{1}(\mathrm{T} 21)$ in the population seemed to decrease while the mean maternal age increased. The NT- values in the T21-affected pregnancies were very low. These data suggested a sequential screening practice: second trimester maternal serum screening was only performed when first trimester NT screening was normal, because the pregnancies with abnormal first trimester NT screening results were already referred for invasive testing.

In Chapter 5, it is explained that both first and second trimester maternal serum screening are useful methods to identify a proportion of pregnancies at matemal age $\geq 35$ years, in which the iatrogenic risk of fetal loss after invasive testing is higher than the risk for fetal aneuploidy. Both methods may be helpful to reduce efficiently the invasive procedures in unaffected pregnancies at advanced maternal age and to stop the evolution of primary invasive tests in Belgium, as described in Chapter 2 .

In Chapter 6, it is reported that the NT measurements of a group of Flemish obstetricians were lower than the NT measurements of ultrasonographers, trained by the Fetal Medicine Foundation (FMF), and also lower than the FMF reference values. The impact of this underestimation was most relevant for women at advanced maternal age, and was less important when maternal serum parameters were combined with ultrasound screening. Standardisation of ultrasound screening methodology is proposed towards optimisation of ultrasound or combined screening for fetal aneuploidy.

In Chapter 7, the results of a survey on the future organisation of first trimester ultrasound screening in Flanders are presented. This survey showed that the majority of chief-obstetricians in the country considered first trimester ultrasound screening a routine prenatal service, to be available in every obstetric 
clinic. They also agreed for participation in a program for ultrasound training and quality control. Following this survey, a project for ultrasound training and quality control has been initiated by the Specialist Centres for Obstetric Ultrasound in Flanders, in collaboration with the FMF.

Chapter 8 presents the screening results of first trimester combined screening, using the NT measurements as discussed in Chapter 6. As expected, the detection of fetal $\mathrm{T} 21$ was less than in the reported single centre studies using FMF scanning criteria. However, the screening results were close to the reported results of multicenter studies using FMF scanning criteria or using population- or performer- specific NT medians. Easy access to screening and maternal serum parameters accounted for the majority of T21 detections in the AML screening program.

In Chapter 9, the implementation of NT measurements into the AML fetal aneuploidy screening algorithms is investigated. Relative to a median NT value at every stage of gestation, NT measurements can be expressed as a product (MoM) or a difference (Delta). The median NT values can be obtained from the FMF- reference curve or can be population- or performer-specific. It is concluded that there are no arguments for replacing $\operatorname{LogNT}-\mathrm{MoM}$ values by Delta-NT values in the AML screening program, but using performer-specific NT-medians instead of the fixed FMF reference range would allow for high quality and easy accessible screening tests.

Chapter 10 summarizes the organisation of fetal aneuploidy screening practice in Flanders and/or Belgium between 1995 and 2003. The overall provision of screening in Belgium was good. The quality of screening methods was discussed in the previous chapters, using the AML screening program as an example. The registration of fetal aneuploidy screening activities in Flanders 
and Belgium was poor. Some recommendations are made towards improvement of future screening practice.

In Chapter 11, the answers to the 4 basic questions of this thesis are summarized:

1. Maternal serum screening methods in the AML screening program are adequate, but performance of ultrasound screening methods is suboptimal.

2. Combined first trimester screening is currently the best option for screening the individual patient and also for screening the population. New methods however will further improve future screening results.

3. A reduction of invasive procedures in unaffected pregnancies without major loss of T21 detections can be achieved by an increase of maternal serum screening at advanced maternal age and by a reduction of two step sequential screening. Future screening results might improve after (1) optimisation of ultrasound screening, (2) introduction of integrated first and second trimester screening and (3) reorganisation of screening services in a system of contingent screening.

The current registration of fetal aneuploidy screening activities in Flanders or Belgium is poor, and insufficient to guide and survey the future organisation of screening. 
192 
Chapter 13

\section{Samenvatting}


Zowel in België als in Nederland, en waarschijnlijk ook nog in vele andere landen, blijkt het niet zo eenvoudig om nieuwe technieken ter opsporing van foetale afwijkingen in de bevolking snel en efficiënt te introduceren. De meeste belemmeringen worden veroorzaakt door de organisatie van de gezondheidszorg in deze landen. Uitgaande van het screeningsprogramma ter opsporing van fetaal Down Syndroom (Trisomie 21), georganiseerd door het Algemeen Medisch Laboratorium in Antwerpen, biedt dit proefschrift een overzicht van de huidige screeningsmethodes in Vlaanderen, België. Dit onderzoek toont aan hoe belangrijk het is om nauwkeurig te evalueren of nieuwe technieken kwalitatief goed worden uitgevoerd. Door de zwakke en sterke punten in kaart te brengen, kan de verdere implementatie en uitbouw van deze technieken in cle dagelijkse klinische praktijk worden verbeterd, hetgeen nuttig is voor de organisatie van de prenatale screening.

In Hoofdstuk 1 wordt de geschiedenis van trisomiescreening besproken, evenals de verschillende screeningsmethodes: vruchtwaterpuncties of vlokkentesten uitgevoerd bij oudere zwangeren (primaire puncties $\rangle$, screening $\mathrm{dmv}$ bloedafnames (serumscreening) en screening $\mathrm{dmv}$ echoscopie (nekplooimeting). De risico's van vruchtwaterpuncties en vlokkentesten worden besproken en vergeleken. De methodiek, efficiëntie en klinische toepassing van de serumscreening en nekplooimeting worden besproken en vergeleken en uitgelegd wordt hoe beide methodes op een efficiënte wijze het aantal primaire puncties in normale zwangerschappen op oudere leeftijd kunnen reduceren. Enkele verschillen in de gezondheidszorg en trisomiescreening in België en Nederland worden besproken.

In Hoofdstuk 2 wordt de evolutie beschreven van de serumscreening en de puncties in België in de periode 1995 - 2001, uitgaande van de data van het Rijksinstituut voor de Ziekte- en Invaliditeitsverzekering (RIZIV) en van het ziekenfonds Christelijke Mutualiteiten (CM). Aangetoond wordt dat de stijging 
van de puncties, die in deze periode plaatsvond, vooral het gevolg is van een toename van het aantal primaire puncties op oudere leeftijd. Deze observatie is duidelijk anders dan verwacht uit de literatuurgegevens (zie Hoofdstuk 1) en vormde de aanzet tot deze thesis. Hierin worden 4 concrete vragen geformuleerd:

1. Welke zijn de verschillende methodes voor trisomiescreening, die momenteel in Vlaanderen/België worden toegepast, en stemt hun kwaliteit overeen met de gegevens uit de literatuur?

2. Kan de optimale screeningsmethode worden aangeduid, zowel van het standpunt van de individuele patiënt als van de populatie?

3. Is het mogelijk om bepaalde strategieën uit te stippelen, die de detectie van T21 verbeteren en die de puncties in normale zwangerschappen reduceren?

4. Is de huidige registratie van screeningsactiviteiten in Vlaanderen/België voldoende om een goed overzicht te kunnen bieden, om de kwaliteit te beoordelen, en om de uitbouw van verdere screening te sturen en coördineren?

In Hoofdstuk 3 worden enkele van de basisprincipes van trisomiescreening besproken, die relevant waren voor de ontwikkeling van de algoritmes voor het screeningsprogramma van het Algemeen Medisch Laboratorium (AML) in Antwerpen, België. Het AML register is één van de twee Belgische databanken voor trisomiescreening, die in de literatuur worden beschreven. De methodiek van dit screeningsprogramma wordt uitvoerig besproken. Ook is aangegeven hoe deze methodes zijn gevalideerd, hoe kwaliteitscontrole werd toegepast en hoe het programma werd geoptimaliseerd.

In Hoofdstuk 4 worden de resultaten van 10 jaar serumscreening besproken: de efficiëntie van bloedtesten uitgevoerd rond 16 weken zwangerschapsduur bleek volledig vergelijkbaar te zijn met de gegevens uit de literatuur. Het aantal testen 
uitgevoerd bij zwangeren vanaf 35 jarige leeftijd was evenwel minder dan verwacht volgens de leeftijdsdistributie van de totale populatie zwangeren in Vlaanderen. Dit verschil werd toegeschreven aan het uitvoeren van primaire puncties bij een groot aantal oudere zwangeren, zonder voorafgaande serumscreening. De resultaten van serumscreening rond 16 weken, uitgevoerd na de introductie van de nekplooimeting als bijkomende screeningsparameter, waren moeiligk interpreteerbaar vanwege de kleine aantallen. Vanaf 1999 leek er een daling op te treden van $\mathrm{T} 21$ in de populatie, die serumscreening onderging, hoewel de gemiddelde maternale leeftijd toenam. De nekplooimetingen in de T21 zwangerschappen waren daarenboven gelijk aan deze in de normale zwangerschappen. Deze resultaten werden toegeschreven aan het uitvoeren van een 2-staps sequentiele screeningstechniek: een serumscreening op 16 weken werd slechts toegepast bij die zwangeren die op 12 weken een normale nekplooimeting hadden, want diegenen met een abnormale echoscopie werden meteen verwezen voor een punctie.

In Hoofdstuk 5 wordt aangetoond dat zowel de serumscreening op 16 weken als deze op 12 weken zwangerschapsduur geschikt zijn om zwangerschappen vanaf de leeftijd van 35 jaar te identificeren, waarvan het risico op $\mathrm{T} 21 \mathrm{kleiner}$ is dan de kans op zwangerschapsverlies als het gevolg van een punctie. Beide methodes kunnen dus van nut zijn ter reductie van puncties in normale zwangerschappen op oudere leeftijd en om de evolutie van de puncties, zoals beschreven in Hoofdstuk 2, te stoppen.

In Hoofdstuk 6 is werd de toepassing geëvalueerd van de echoscreening $\mathrm{dmv}$ de meting van de foetale nekplooi. Vlaamse gynaecologen bleken de nekplooi sytematisch kleiner te meten dan de echografisten, getraind door de Fetal Medicine Foundation (FMF), en hun waarden vielen ook kleiner uit dan de FMF- referentiewaarden. De impact van deze metingen op de screeningsresultaten was het meest uitgesproken bij oudere zwangeren en 
verminderde na combinatie met serumscreening. Het standaardiseren van de echoscopische methode om nekplooimetingen uit te voeren werd voorgesteld ter optimalisatie van de resultaten van het screeningsprogramma.

De resultaten van een enquête over de toekomstige organisatie van echoscreening in Vlaanderen zijn gepresenteerd in Hoofdstuk 7. De meerderheid van de ondervraagde Vlaamse diensthoofden Verloskunde beschouwden echoscreening als een onderdeel van de routine zwangerschapszorg, die beschikbaar hoort te zijn in elke Vlaamse verloskundige kliniek. Om een zo optimaal mogelijke kwaliteit te garanderen, verklaarden ze zich akkoord tot deelname aan een programma ter training en kwaliteitscontrole van de methodiek van echoscreening. Gevolg gevend aan de resultaten van deze enquête, hebben de Vlaamse Centra voor Verloskundige Echografie gezamenlijk een project gestart dat voorziet in deze training en kwaliteitscontrole van echoscreening. Dit project loopt in samenwerking met de FMF.

In Hoofdstuk 8 zijn de resultaten beschreven van trisomiescreening dmv een combinatie van serum en echo, uitgevoerd rond een zwangerschapsduur van 12 weken. De nekplooimetingen, gebruikt voor deze screeningsmethode, werden reeds in hoofdstuk 6 beschreven. Zoals verwacht was de detectie van T21 lager dan deze gerapporteerd door individuele centra, die screenen volgens de FMF. methodiek. Anderzijds lag deze detectie erg dicht bij de gerapporteerde resultaten van multi-centrische screeningsprogramma's, zowel diegenen die gebruik maakten van de FMF-methodiek en -referentiewaarden als diegenen die gebruik maakten van eigen referentiewaarden. Een gemakkelijke toegang tot screening en serumscreening waren verantwoordelijk voor de meerderheid van de T21-detecties in het AML-screeningsprogramma. 
In Hoofdstuk 9 zijn de verschillende methodes vergeleken om nekplooimetingen te implementeren in de AML- algoritmes voor trisomiescreening. Deze metingen kunnen worden uitgedrukt als een product van een gemiddelde $(\mathrm{MoM})$ of als een verschil ten opzichte van een gemiddelde (Delta). Het gemiddelde dat als referentie wordt gebruikt kan afkomstig zijn van de FMF-referentiecurve of kan een populatiespecifiek gemiddelde zijn. Het bestudeerde AML-screeningsprogramma gebruikte MoM-waarden, gerefereerd naar de FMF-referentiecurve. Er worden geen argumenten weerhouden om deze MoM-waarden te vervangen door Delta-waarden, maar het gebruiken van populatiespecifieke referentiewaarden in plaats van de FMF-referentiecurve zal toelaten kwaliteitsvolle risicoberekeningen te combineren met een goed toegankelijke screening.

In Hoofdstuk 10 wordt de dagelijkse praktijk van trisomiescreening in Vlaanderen/België beschreven in de periode 1995 - 2003. Het aanbod en de toegankelijkheid van de verschillende screeningsmethodes was goed. Met het AML-screeningsprogramma als voorbeeld, werd de kwaliteit van de verschillende screeningsmethodes reeds uitgebreid besproken in de voorgaande hoofdstukken. De registratie van de verschillende stappen in het screeningsproces was zwak en onvoldoende om de verdere organisatie van trisomiescreening in het land te sturen en te coördineren. Enkele aanbevelingen werden geformuleerd om de screeningsactiviteiten te verbeteren en te optimaliseren.

Hoofdstuk 11 geeft een antwoord op de 4 gestelde vragen van dit proefschrift:

1. De methodiek en de resultaten van de serumscreening in het AMLscreeningsprogramma zijn goed, maar de methodiek van de echoscreening is suboptimaal.

2. De combinatie van serumscreening en echoscreening, beiden uitgevoerd rond 12 weken zwangerschapsduur, is momenteel de beste optie tot 
screening van de individuele zwangere en van de populatie. In de toekomst kunnen evenwel nieuwe methodes deze resultaten merkelijk verbeteren.

3. Een reductie van puncties in normale zwangerschappen, met beperkte impact op de detectie van T21, kan worden gerealiseerd door een toename van serumscreening op oudere leeftijd en door een reductie van 2-staps sequentiële screening. De eindresultaten van de screening zouden eventueel nog kunnen verbeteren door (1) optimalisatie van de echoscreening, (2) door Integratie van de screeningsmethodes, uitgevoerd op 12 weken, met deze uitgevoerd op 16 weken en (3) door reorganisatie van het screeningsaanbod in een systeem van Contingent screening.

4. De huidige registratie van de screeningsactiviteiten in Vlaanderen/België is zwak en onvoldoende om de verdere uitbouw van trisomiescreening te sturen en te coördineren. 


\section{Nawoord en dank}

Over het tijdsverloop van deze thesis werden me bij herhaling steeds dezelfde vragen gesteld:

1. Waarom wil een arts, werkzaam in een niet - universitaire praktijk, een thesis maken?

2. Waarom wordt een thesis over een Vlaams-Belgisch onderwerp afgelegd in een Nederlandse universiteit?

3. Waarom werd "Screenen naar foetaal Down syndroom" gekozen als onderwerp voor een thesis?

Ik onderneem een poging om deze vragen zinvoll te beantwoorden.

Waarom wil een arts, werkzaam in een niet - universitaire praktijk, een thesis maken?

Bloed kruipt waar het niet gaan kan. Van kindsbeen af had ik reeds een bijzondere interesse voor wetenschappelijke onderwerpen, wat zich vertaalde in de keuze om geneeskunde te gaan studeren. Tijdens deze studies, en ook als specialist in opleiding, bleef de onderzoeksmicrobe kriebelen en werd me twee maal de mogelijkheid geboden deze interesse verder uit te werken in fundamenteel, basiswetenschappelijk onderzoek. Het klinische werk en de dagelijkse contacten met patiënten leken me op dat ogenblik evenwel aanlokkelijker dan het zuivere Jaboratoriumwerk, en ik verkoos om in de kliniek te stappen. Gedurende bijna tien jaar medische praktijk werd ik meer en meer geconfronteerd met het groeiende probleem om, conform de huidige standaarden van geneeskunde, privé-patiëntenzorg en continue klinische beschikbaarheid te blijven combineren met het up-to-date blijven met de snelle ontwikkelingen binnen elke tak van de geneeskunde en van de VerloskundeGynaecologie. Dit is een dilemma dat zich op dit ogenblik stelt voor vele artsen en gynaecologen in Vlaanderen en België, waar de algemene gezondheidszorg een sterk privé karakter heeft, gekend en gewaardeerd in vele andere landen, 
doch zeer "opslorpend" voor de betrokken gezondheidswerkers. De beste manier om uit te zoeken of men (lees "ikzelf") in het klinisch werk nog op het goede spoor zat, was het kritisch nakijken en beoordelen van de resultaten van gerealiseerde klinische activiteiten. Deze vraagstelling was de grondmotivatie voor deze thesis.

Waarom wordt een thesis over een Vlaams-Belgisch onderwerp afgelegd in een Nederlandse universiteit?

Klinische audit, vanuit een pur medisch-wetenschappelijk perspectief, is minder gebruikelijk in de Vlaamse en Belgische geneeskundige praktijk, doch is veel meer ingeburgerd in de Nederlandse en Angelsaksische gezondheidszorg.

De voornaamste reden evenwel om voor Nederland te kiezen was de behoefte aan een neutrale en ongekleurde supervisie van een onderzoeksproject dat vele verloskundige diensten overkoepelde, elk met hun eigen achtergrond en strekking en geaffilieerd met de verschillende Vlaamse universiteiten. Enkele jaren geleden ontmoette ik Jan Nijhuis tijdens de cursus "Diploma in Fetal Medicine" in Londen, waar hij één van de lectoren was. Ook al was deze ontmoeting eerder vluchtig en kort, zijn open en vriendelijke karakter lieten een indruk na. Daarenboven werd hij door menige aanwezige gerespecteerd voor zijn persoonlijke visie op diverse aspecten van de huidige verloskunde en voor zijn subtiele en diplomatieke wijze om conflicten aan te pakken en op te lossen. Deze eigenschappen, samen met het feit dat de afstand tussen Genk en Maastricht amper 30 kilometer bedraagt, maakten hem de geknipte persoon om dit werk te superviseren. Hij was onmiddellijk akkoord om deze taak op zich te nemen. Vanuit het concept "Think future, think Europe" hoopten we enkele gegevens te kunnen bekomen, die van nut zouden kunnen zijn zowel in België als in Nederland. Tenslotte was er voor mij ook nog een persoonlijke motivatie om naar Maastricht te gaan. Mijn overgrootvader verhuisde op het einde van de $19^{\circ}$ eeuw van Berg-en ter Bleyt bij Maastricht naar Diepenbeek bij Genk. Het 
aanknopen van de relaties met Nederlands Limburg kreeg alzo iets van het terug opzoeken van een oude bekende, van een terugkeer naar de eigen roots.

\section{Waarom werd "Screenen naar foetaal Down syndroom" gekozen als onderwerp voor een thesis?}

Het ontwikkelen en verfijnen van testen ter detectie van ongeboren baby's met Down syndroom wordt wel eens geïnterpreteerd als een moderne "heksenjacht", als een poging om via interventies ervoor te zorgen dat personen met deze aandoening niet meer in de maatschappij terecht kunnen komen. Dit is niét de drijfveer achter deze thesis. In familiale kring ben ik persoonlijk geconfronteerd met de gevolgen van mentale en sociale handicap. Dit heeft me vooral geleerd te relativeren en te respecteren. Respect is een sleutelwoord geworden in de samenwerking met anderen en in het dagelijkse leven. "De erkenning van waarden is maar mogelijk door kennis van en eerbied voor de andere waarden."

Dr. Amaat Van Waes was bijna 60 jaar toen hij me de beginselen van de verloskunde bijbracht. Hij schetste de evolutie van de obstetrie als volgt: "Van de preventie van maternale sterfte, via de preventie van perinatale sterfte naar het optimaliseren van de levenskwaliteit van elke pasgeborene door reductie van de perinatale morbiditeit". In Vlaanderen is de perinatale sterfte nooit zo laag geweest als nu, en men verwacht nog slechts een beperkte en geleidelijke verdere daling. Perinatale morbiditeit wordt meer en meer naar voor geschoven als parameter om verloskundige zorgen te meten. De technieken voor opsporing van aangeboren aandoeningen ontgroeien stilaan de kinderschoenen en vinden geleidelijk hun weg naar de dagelijkse verloskundige praktijk. Deze thesis wil hiertoe een steentje bijdragen. Op deze wijze is dit werk ook een gepast eerbetoon aan de man die me de verloskunde heeft leren ontdekken.

Een laatste, en zeker niet te onderschatten, reden om "Screenen naar Down syndroom" als thesisonderwerp te kiezen was de vriendschappelijke samenwerking met het Algemeen Medisch Laboratorium AML te Antwerpen, zoals deze was gegroeid in de voorbije jaren. De bijdrage die de mensen van 
AML hebben geleverd aan dit werk is van onnoemelijke waarde. Het waren tenslotte zij die het initiatief genomen hebben tot het opstarten van een screeningsprogramma, die het doorzettingsvermogen hadden om niet gerapporteerde gegevens te blijwen opvragen en zoveel als mogelijk te verzamelen, die de gedrevenheid aan de dag legden om voortdurend de resultaten te evalueren en te verbeteren, het waren zij die in de loop der jaren een register over Down syndroom screening hebben opgebouwd dat in Vlaanderen zijns gelijke niet heeft. Ik dank hen hiervoor oprecht. Deze thesis is er dankzij hun werk!

Ik dank vooral ook diegenen die me naw aan het hart liggen en me de voorbije jaren hebben gesteund en gestimuleerd: mijn echtgenote Ingrid en mijn kinderen Jan, Gert en Leen. Ik dank ook mijn oude moeder, die voor alles toch de basis heeft gelegd. Ook nog een hartelijk woord van dank aan mijn collegae binnen het departement Verloskunde - Gynaecologie van het Ziekenhuis Oost Limburg in Genk, die me temidden van alle drukke klinische activiteiten toch de nodige ruimte en tijd gegund hebben om aan deze thesis te werken.

Tenslotte nog dit: bijgaande figuur vond ik onlangs in een oude atlas over verloskundige echografie "Ultraschalldiagnostik in Geburtshilfe und Gynäkologie: Lehrbuch und Atlas. Eds: A. Kratochwil, H. Husslein. Georg Thiene Verlag, Stutgant, 1968".

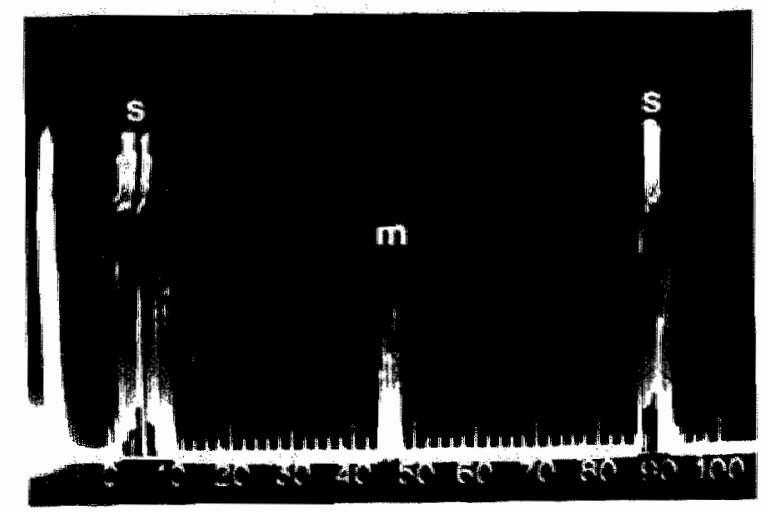


Het toont één van de eerste methodes om met ultrageluid de bipariètale diameter (BPD) van het foetale hoofd te meten. De auteurs noemden deze techniek een ware revolutie in de verloskunde, gezien zij toeliet de foetale biometrie tot op de millimeter nauwkeurig te bepalen. Deze publicatie is slechts 37 jaar oud. In deze thesis wordt veel gesproken over het belang en de relativiteit van echografische metingen tot op een tiende millimeter nauwkeurig. Ik hoop dat over 37 jaar iemand dit werk nog eens kan inkijken en mijmert over de evolutie die sindsdien heeft plaatsgevonden ...

Wilfried Gyselaers

Genk, 15 augustus 2005. 


\section{Curriculum vitae}

De auteur van dit proefschrift werd geboren op 30 juli 1963 te Hasselt, Belgie. $\mathrm{Na}$ het beëindigen van de lagere school in de St.-Servatiusschool te Diepenbeek, volgde hij de studierichting Grieks-Latijn en later GrieksWiskunde aan het St.-Jozefscollege te Hasselt. Hij startte zijn studies geneeskunde in 1981 aan het Limburgs Universitair Centrum te Diepenbeek om ze in 1988 met Grote Onderscheiding te voltooien aan de Katholieke Universiteit te Leuven. Tijdens deze studies deed hij in 1983 en 1984 gedurende de zomervakanties een laboratoriumstage in het Rega-Instituut te Leuven onder leiding van Professor A. Billiau. Zijn aandeel in een studieproject rond Papillomavirussen in humane weefsels werd neergeschreven in zijn eindwerk getiteld: "De betekenis van Humaan Papillomavirus in cervicale lesies: een model van carcinogenese van HPV-geassocieerde letsels."

Zijn opleiding tot specialist in de gynaecologie-verloskunde gebeurde aan de Katholieke Universiteit te Leuven onder supervisie van Professor A. Van Assche. Tijdens deze opleiding was hij 3 jaar werkzaam in het Universitair Ziekenhuis Gasthuisberg te Leuven, waar hij o.m. verloskundige echografie leerde bij Prof. K. Vandenberghe. Hij was hier ook aktief betrokken in een studieproject rond Thyrotropin Releasing Hormoon en foetale longmaturatie. Nadien werkte hij 1 jaar in Watford General Hospital te Londen onder supervisie van Mr. V. Lewis. Tijdens die periode nam hij regelmatig deel aan de casusbesprekingen hoog-risico verloskunde onder leiding van Prof. J. Steer in het West London Hospital van de Charing Cross and Westminster Medical School, University of London, en nam succesvol deel aan het MRCOG- examen Part 1 . Hij beëindigde zijn opleiding tot specialist in het Imeldaziekenhuis te Bonheiden bij Dr. P. Vermijlen.

Hij startte in 1993 een klinische praktijk als algemeen gynaecoloog in het Ziekenhuis Oost Limburg te Genk, België. Hier werd hij in 1998 verantwoordelijk voor de uitbouw van een nieuw opgerichte dienst voor de 
opvang van risicozwangerschappen ("Maternal Intensive Care"). Nadat hij in 1999 succesvol het theoretisch examen aflegde voor het behalen van het "Diploma in Fetal Medicine" bij Prof. K. Nicolaides te London, bouwde hij ook de dienst Verloskundige Echografie in het ZOL verder uit.

Hij is gehuwd met Ingrid Timmerman, verpleegkundige intensieve zorgen, en heeft 3 kinderen: Jan ( $\left.{ }^{\circ} 1989\right)$, Gert $\left({ }^{\circ} 1990\right)$ en Leen $\left({ }^{\circ} 1993\right)$.

Dit proefschrift werd aangevat in juli 2002. 


\section{Publicaties}

Gyselaers W, Vereecken A, Van Herck E, Straetmans D, de Jonge E, Ombelet W, Nijhuis J. (2005) Population screening for fetal trisomy 21: easy access to screening should be balanced against a uniform ultrasound protocol. Prenat Diagn (in press).

Gyselaers W, De Catte L, Witters I, de Jonge E, Ombelet W, Nijhuis J. (2005) Survey on the future organisation of first trimester ultrasound screening in Flanders, Belgium. Prenat Diagn 25:518-9.

Gyselaers W, Vereecken A, Van Herck E, Straetmans D, de Jonge E, Ombelet W, Nijhuis J. (2004) Audit on nuchal translucency thickness measurements in Flanders, Belgium: a plea for methodological standardization. Clitrasound Obstet Gynecol 24:511-5.

Gyselaers W, Vereecken A, Van Herck E, Straetmans D, de Jonge E, Ombelet W, Nijhuis J. (2004) Single-step maternal serum screening for trisomy 21 in the era of combined or integrated screening. Gynecol Obstet Invest 58:221-4.

Gyselaers W, Vereecken A, Van Herck E, Straetmans D, de Jonge E, Ombelet W, Nijhuis J. (2004) Screening for fetal trisomy 21 in Flanders: a 10years review of 40490 pregnancies screened by maternal serum. Eur $J$ Obstet Gynecol Reprod Biol 1 15:185-9.

Gyselaers W, Spitz B, de Zegher F, Van Ballaer P, Hanssens M, Van Assche FA. (1992) Effects of thyrotropin-releasing hormone on fetal heart rate. Lancet 339:1417.

Gyselaers W, Vansteelant L, Spitz B, Odendaal HJ, Van Assche FA. (1991) Do biphasic uterine contractions imply poor uterine function? Eur J Obstet Gynecol Reprod Biol 42:111-4.

Ombelet W, Peeraer K, De Sutter P, Gerris J, Bosmans E, Martens G, Ruyssinck G, Defoort P, Molenberghs G, Gyselaers W. (2005) Perinatal outcome of ICSI pregnancies compared with a matched group of natural 
conception pregnancies in Flanders (Belgium): a cohort study. Reprod Biomed Online 11:244-53.

Ombelet W, Cadron I, Gerris J, De Sutter P, Bosmans E, Martens G, Ruyssinck G, Defoort P, Molenberghs G, Gyselaers W. (2005) Obstetric and perinatal outcome of 1655 ICSI and 3974 IVF singleton and 1102 ICSI and 2901 IVF twin births: a comparative analysis. Reprod Biomed Online 11;76-85.

Verswijvel G, Gyselaers W, Grieten M, Van Robaeys J, Palmers Y. (2002) Omphalocoele: prenatal MR findings. JBR-BTR 85:200-2.

Verswijvel G, Grieten M, Gyselaers W, Van Holsbeke C, Vandevenne J, Horvath M, Gelin G, Palmers Y. (2002) MRI in the assessment of pregnancy related intrauterine bleeding: a valuable adjunct to ultrasound? JBR-BTR 85:189-92.

Witters I, Vermeesch JR, Gyselaers W, Fryns JP. (2002) Distinct prenatal ultrasonographic cranial and cerebral findings in terminal deletion of the short arm of chromosome 1. Prenat Diagn 22:831-4.

Witters I, Theyskens C, van Hoestenberghe R, Sieprath P, Gyselaers W, Fryns JP. (2001) Prenatall diagnosis of non-obstructive megacystis as part of the megacystis-microcolon-intestinal hypoperistalsis syndrome with favourable postnatal outcome. Prenat Diagn 21: 704-6.

Ombelet W, Bosmans E, Janssen M, Cox A, Vlasselaer J, Gyselaers W, Vandeput H, Gielen J, Pollet H, Maes M, Steeno O, Kruger T. (1997) Semen parameters in a fertile versus subfertile population: a need for change in the interpretation of semen testing. Human Reprod 12:98793.

Grubben C, Gyselaers W, Moerman P, Vandenberghe K, Fryns JP. (1990) The echographic diagnosis of fetal akinesia. A challenge towards etiological diagnosis and management. Genet Couns 1:35-40. 


\section{Abstracts}

Gyselaers W, Vereecken A, Nijhuis J. The organisation of fetal aneuploidy screening in Flanders, Belgium. $15^{\circ}$ Congres Infertiliteit Gynaecologie en Obstetrie, Rotterdam De Doelen, 6-8/4/5. In: Slager E, Fauser B, van Geijn $H$, Brölmann $H$, Vervest $J$ (eds). Gynaecology, obstetrics and reproductive medicine in daily practice. Internat Congress Series 1279; (in press).

Gyselaers W, Vereecken A, Van Herck E, Straetmans D, de Jonge E, Ombelet W, Nijhuis J. Audit on fetal Down's Syndrome screening in a Belgian population between 1992 and 2004. XIX European Congress of Perinatal Medicine, Athens 13-16/10/2004. I Maternal Fetal Neonat Med 2004;16(S1):44.

Gyselaers W. Eerste trimester screening in Vlaanderen. Euregio Ultrasound Symposium OB/GYN 3, Maastricht 17/6/2004.

Gyselaers W. Basale echocardiografie: eenvoudiger met het gebruik van 6 standaard-doorsnedes? Ultrasound Symposium OB/GYN 2, Maastricht 19/6/2003.

Gyselaers W, Van Holsbeke C, Druwé A, de Jonge E, Ombelet W. Evaluation of a protocol for ultrasonic systematic morphological foetal examination in an obstetric screening clinic. Euroson 2001, Edinburgh 1114/12/2001.

Gyselaers W, Van Holsbeke C, Hinoul P, de Jonge E, Ombelet W. Doppler study of intracervical blood flow in the third trimester of pregnancy. Research and developments in Fetal Medicine, London, 30/8 1/9/2001.

Verswijfel G, Vandevenne J, Grieten M, Gyselaers W, Van Holsbeke C, Horvath M, Gelin G, Palmers Y. MRI in the assessment of pregnancy related intrauterine bleeding: a valuable adjunct to ultrasound? World Congress of Medical Imaging, San Francisco, august 2001. 
Gyselaers W, Spitz B, de Zegher F, Van Assche FA. Fetall lung maturation and TRH. West London Hospital, Charing Cross and Westminster Medical School, University of London. 17 june 1992. 
